\title{
Monotonicity and Bayes-Nash implementation
}

Citation for published version (APA):

Wolf, S. (2009). Monotonicity and Bayes-Nash implementation. [Doctoral Thesis, Maastricht University]. Datawyse / Universitaire Pers Maastricht. https://doi.org/10.26481/dis.20091215sw

Document status and date:

Published: 01/01/2009

DOI:

10.26481/dis.20091215sw

Document Version:

Publisher's PDF, also known as Version of record

\section{Please check the document version of this publication:}

- A submitted manuscript is the version of the article upon submission and before peer-review. There can be important differences between the submitted version and the official published version of record.

People interested in the research are advised to contact the author for the final version of the publication, or visit the DOI to the publisher's website.

- The final author version and the galley proof are versions of the publication after peer review.

- The final published version features the final layout of the paper including the volume, issue and page numbers.

Link to publication

\footnotetext{
General rights rights.

- You may freely distribute the URL identifying the publication in the public portal. please follow below link for the End User Agreement:

www.umlib.nl/taverne-license

Take down policy

If you believe that this document breaches copyright please contact us at:

repository@maastrichtuniversity.nl

providing details and we will investigate your claim.
}

Copyright and moral rights for the publications made accessible in the public portal are retained by the authors and/or other copyright owners and it is a condition of accessing publications that users recognise and abide by the legal requirements associated with these

- Users may download and print one copy of any publication from the public portal for the purpose of private study or research.

- You may not further distribute the material or use it for any profit-making activity or commercial gain

If the publication is distributed under the terms of Article $25 \mathrm{fa}$ of the Dutch Copyright Act, indicated by the "Taverne" license above, 


\section{Monotonicity}

and

Bayes-Nash Implementation

Sascha Wolf 
(c) Sascha Wolf, 2009

All rights reserved. No part of this publication may be reproduced, stored in a retrieval system, or transmitted in any form, or by any means, electronic, mechanical, photocopying, recording or otherwise, without the prior permission in writing from the author.

This book was typeset by the author using $\mathrm{LT}_{\mathrm{E}} \mathrm{X}$.

Published by Universitaire Pers Maastricht

ISBN: 978-90-5278-899-9

Printed in The Netherlands by Datawyse Maastricht 


\title{
Monotonicity
}

\author{
and

\section{Bayes-Nash Implementation}

\section{PROEFSCHRIFT}

ter verkrijging van de graad van doctor

aan de Universiteit Maastricht,

op gezag van de Rector Magnificus,

Prof. mr. G.P.M.F. Mols,

volgens het besluit van het College van Decanen, in het openbaar te verdedigen op

dinsdag 15 december 2009 om 12.00 uur

door

Sascha Wolf

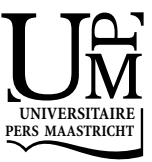




\section{Promotor:}

Prof. dr. R.J. Müller

\section{Copromotor:}

Dr. Ir. A. Perea y Monsuwé

\section{Beoordelingscommissie:}

Prof. dr. H.J.M. Peters (voorzitter)

Dr. A.J. Vermeulen

Prof. dr. R.V. Vohra (Northwestern University)

Dit onderzoek werd gefinancieerd door de Maastricht Research School of Economics of Technology and Organizations (METEOR). 


\section{Acknowledgements}

I owe a debt of heartfelt gratitude to a number of people, without whose continued support I would neither have started nor finished the work on this dissertation ... especially finished. First and foremost I would like to thank my supervisors Rudolf Müller and Andrés Perea for always keeping their doors open and helping me with their advice and guidance.

I thank the members of the Degree Committee, Hans Peters, Dries Vermeulen and Rakesh Vohra, for taking the time to read and referee this thesis. Rakesh Vohra also deserves thanks for providing comments at earlier stages of the work. I am grateful to all colleagues who provided helpful discussions and comments at various conferences and workshops.

I extend my gratitude to all my colleagues at the Department of Quantitative Economics for providing a very pleasant working atmosphere. In particular, I would like to thank Karin van den Boorn and Haydeé Hallmanns for their administrative support, Stephan Smeekes for his help with the Dutch summary of this thesis and Jean-Pierre Urbain for his guidance during my Econometrics study. I especially thank my colleague and friend Christian Gengenbach with whom I started studying in Maastricht 10 years ago.

Last but not least I thank my friends and family. I would like to express my largest gratitude to my parents Ernst and Renate Wolf and my grandmother Lotte Reichstein. Thank you for your unconditional love.

Sascha Wolf

Maastricht, October 2009 



\section{Contents}

\section{Acknowledgements}

1 Introduction 1

1.1 Mechanism Design . . . . . . . . . . . . . . . . . 1

1.1.1 Some Basic Concepts . . . . . . . . . . . . . . . . . 3

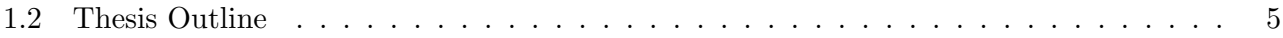

2 Monotonicity and One-Dimensional Types 9

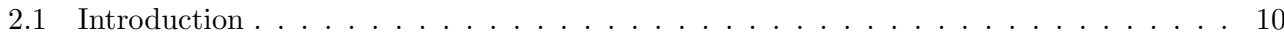

2.1 .1 Related Work . . . . . . . . . . . . . . . . . . . . . . . 10

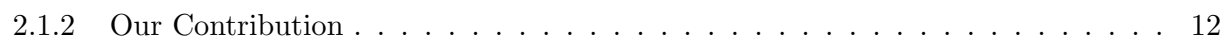

2.2 Model \& Definitions . . . . . . . . . . . . . . . . . . . . . . . . . . . . 14

2.3 A Network Interpretation $\ldots \ldots \ldots \ldots \ldots \ldots$

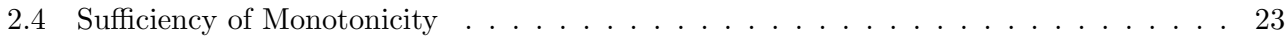

2.5 Payment Rules . . . . . . . . . . . . . . . . . . . . . . . 25

2.5.1 Example Setting: Concave Valuation Functions . . . . . . . . . . . . . . . . 34

2.5.2 Example Setting: Discrete Type Spaces . . . . . . . . . . . . . . . . . . . 36

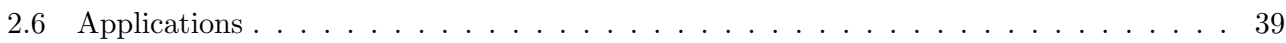

2.6 .1 Single, Indivisible Item . . . . . . . . . . . . . . . . . 40

2.6.2 Multiple Units of a Homogenous Good . . . . . . . . . . . . . . . . 43

2.6 .3 Commonly Ranked Items $\ldots \ldots \ldots \ldots$ 
3 Monotonicity and Multi-Dimensional Types $\quad 51$

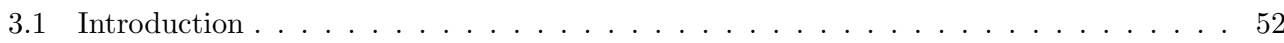

3.1 .1 Related Work . . . . . . . . . . . . . . . . . 53

3.1 .2 Our Contribution . . . . . . . . . . . . . . . . . . . . 54

3.2 Model \& Definitions . . . . . . . . . . . . . . . . . . . . . 57

3.3 A Network Interpretation . . . . . . . . . . . . . . . . . . 62

3.4 Sufficiency of Monotonicity \& Path Independence . . . . . . . . . . . . . . . 66

3.4 .1 Linear Valuation Functions . . . . . . . . . . . . . . . . . . . 74

3.5 Payment Rules . . . . . . . . . . . . . . . . . . . . . . 77

3.6 Applications . . . . . . . . . . . . . . . . . . 83

3.6 .1 Interdependent Valuations . . . . . . . . . . . . . . . . . 84

3.6.2 Single Item Auction With Externalities . . . . . . . . . . . . . . . . 88

4 Combinatorial Scoring Auctions $\quad 91$

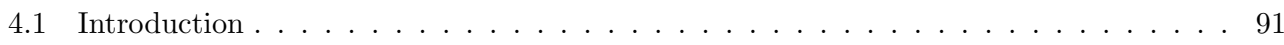

4.1 .1 Related Work . . . . . . . . . . . . . . . . . . . . 93

4.1 .2 Chapter Outline \& Our Contribution . . . . . . . . . . . . . . . . . 95

4.2 Model \& Definitions . . . . . . . . . . . . . . . . . . . . . . . 98

4.2 .1 The Scoring Auction . . . . . . . . . . . . . . . . . . . . . 98

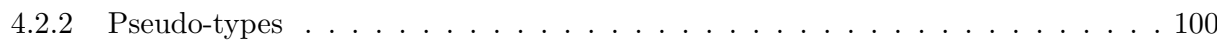

4.3 Pseudo-types Are Sufficient Statistics . . . . . . . . . . . . . . . . . 105

4.4 Technical Note Concerning the Buyer's Utility . . . . . . . . . . . . . . . 114

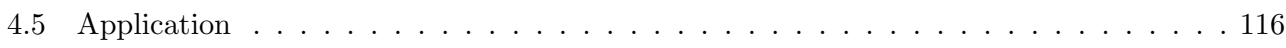

4.5 .1 The VCG Scoring Auction . . . . . . . . . . . . . . . . . . 118

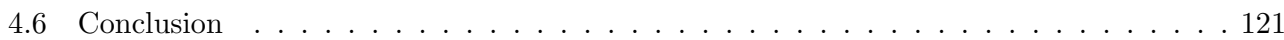

$\begin{array}{ll}\text { Bibliography } & 123\end{array}$

$\begin{array}{ll}\text { Nederlandse samenvatting } & 129\end{array}$

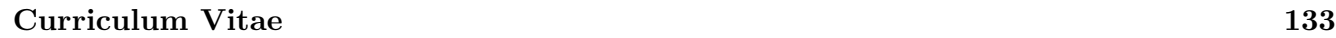




\section{Introduction}

Voting with the rest of the country on who becomes the next president, bidding against other companies on a multi-million dollar highway construction contract from the government or commanding armies in a war - the common factor in all these situations is that the outcome does not solely depend on the actions taken by one participant alone but also on the actions taken by the others. Every day we are faced with such strategic situations the outcome of which depends on our interaction with other people, albeit the outcome is typically of far less import. Think for example of everyday situations like voting with colleagues on where to go for lunch, bidding on a rare Ramones record on Ebay or playing a round of Risk with friends.

Game theory attempts to model people's behavior in such strategic situations mathematically. According to Aumann (1987), we could therefore also call it "interactive decision theory". As he puts it, "game theory is a sort of umbrella or 'unified field' theory for the rational side of social science, where 'social' is interpreted broadly, to include human as well as non-human players (computers, animals, plants)." By drawing on game theoretic analysis and methods we are able to enhance our understanding of vast areas of human and non-human interaction. This makes game theory broadly appealing to various fields of research including economics, political science, computer science and biology. The significance of game theory as a field of study was underlined in 2005 when Robert Aumann and Thomas Schelling received the Nobel Prize in Economics "for having enhanced our understanding of conflict and cooperation through game-theory analysis" (Royal Swedish Academy of Sciences, 2005).

\subsection{Mechanism Design}

The topic of this thesis falls into a sub-field of game theory called mechanism design. In 2007 the Royal Swedish Academy of Sciences recognized the importance of this research area by awarding the Nobel Prize in Economics to Leonid Hurwicz, Eric S. Maskin and Roger B. Myerson "for having laid the foundations of mechanism design theory" (Royal Swedish Academy of Sciences, 2007). 
But what is mechanism design, and why is it such an important field of research? In order to address these questions let us briefly consider a simple but illustrative cake division problem. A mother baked a cake for her two children. Upon receiving the cake both children start fighting over its division, each one trying to score the biggest piece possible. The mother wants to fairly divide the cake in order to end the argument. However, no matter how she cuts the cake, one child will always complain because it feels that it received a smaller piece than the other one. A mechanism that resolves this problem is to let one child divide up the cake and to let the other one choose a piece.

This is a mechanism design problem. In this little example there exists a mechanism designer, the mother, who wants to achieve a certain goal, in this case fair allocation of the cake. But she does not know which allocation would be considered fair. The children are participants with different goals. They do not care about the mother's fairness objective, each child is just interested in getting the biggest piece of cake. Employing the above described mechanism aligns the goals of the designer with those of the participants. For this the children do not have to take the mother's fairness objective into account. Just by following their own myopic interests and adhering to the rules of the mechanism the mother's goal gets satisfied.

Mechanism design theory is concerned with such situations in which some desirable design goals have to be aligned with diverging participants' goals. Typical research questions include: Which design goals are achievable in certain situations and which are not? If a goal is achievable, which mechanism can be used?

Clearly this makes the application of mechanism design theory interesting for many areas of our society, may it be the private or the public sector. Think for example of a company that went bankrupt and gets liquidated. The goal of the trustee in bankruptcy is to gather as much money as possible for the creditors. The potential buyers on the other hand want to maximize their own profits. Which method should the trustee use to sell off the remaining company assets? Or consider a firm that wants to procure production materials. The goal of the firm is to minimize its procurement costs whereas the potential suppliers again aim at maximizing their own profits. Which procurement method should the firm employ? A public sector example is the government trying to allocate public resources such as mining rights or spectrum licenses. The government is concerned with maximizing society's overall social welfare while the competing companies are concerned with the maximization of their own profits. What is a good method of resource allocation? Another public goal is the stop of global warming. How can the government set incentives for the industry to reduce its emission of 
greenhouse gases?

Before we establish how this thesis fits into the field of mechanism design theory, it will be helpful to describe some concepts in more detail.

\subsubsection{Some Basic Concepts}

As established above, when we discuss games, we mean situations in which the interaction of several agents leads to some outcome. Mechanism design is the branch of game theory that concerns itself with designing the rules of games. A game can yield several possible outcomes. Each participating agent has a set of possible actions at his disposal that he can choose from. His choice of action influences the outcome of the game. How exactly is specified by a mechanism which defines the rules of the game. Such a mechanism consists of two rules, an allocation rule and a payment rule. Based on the actions taken by all the agents, the allocation rule determines the outcome of the game. Similarly, the payment rule determines for each agent a payment to make or to receive.

In order to illustrate the above let us consider the auction for a painting as an example. The agents in this auction game are the participating bidders. One possible outcome of the auction is for example that bidder $A$ wins the painting. Another one would be for bidder $B$ to win. The action a bidder can take to influence the auction outcome is to make a money bid for the painting. Based on the bids from all the bidders an allocation rule determines who gets the painting and a payment rule determines how much everyone has to pay.

Four basic auction formats are the English, the Dutch, the first-price sealed-bid and the secondprice sealed-bid auction. The English auction format is the one closest to the Latin root of the word auction, augere - to increase. The price of the painting is successively increased, via the auctioneer or via bids from the bidders, until all but one bidder decided to drop out of the auction. The painting is then allocated to the remaining bidder, and he has to pay the last standing price. As well as in the other three auction formats, the losers pay nothing. The Dutch auction is the converse of the English auction. The name stems from the flower auctions in the Netherlands where this auction format plays a predominant role. In this auction format the auctioneer sets an initial price for the painting which is successively lowered until one bidder accepts the standing price. The painting is then allocated to him at this price. In the first-price sealed-bid auction bidders are asked to submit sealed bids, for example sealed envelopes containing their bids. The painting is allocated to the bidder who submitted the highest bid at a price equal to his bid. The second-price sealed-bid auction works essentially in the same way. The bidders are asked to submit sealed bids, and whoever submitted 
the highest bid wins the painting. However, the price that the winner has to pay is not equal to his own bid but equal to the second highest bid. This auction format was proposed by William Vickrey (1961), therefore it is also commonly referred to as Vickrey auction.

The agents have preferences over the different possible outcomes of the game. An agent's outcome preferences are expressed by his valuation function. Additionally, agents' outcome valuations are also dependent on their types. An agent's type represents information available to him that is influential to his valuation of the different possible outcomes. Throughout this thesis we work in a framework of private information. That is, an agent's type is known to himself but it is not known to any of the other agents. Typically, it is assumed that agents have independent valuations where an agent's valuation depends only on his own type. So an agent's valuation of some outcome would not change if he would get to know any other agent's type. The auction setting presented in Chapter 4 is an example for this. However, the settings presented in Chapters 2 and 3 allow for interdependent valuations. This means that in these settings an agent's valuation for an outcome is not only influenced by his own type but also by the types of the other agents. An agent's outcome valuation together with his payment make up his utility. Throughout the whole thesis we work in a framework of quasi-linear utilities. That is, an agent's utility is his outcome valuation minus his payment. Agents are assumed to be rational, meaning that each agent aims at maximizing his own utility.

Coming back to the painting auction example, an agent's type can for example be a representation of how much he likes the painting. In an independent valuations scenario an agent's valuation for owning the painting would then only depend on how much he likes the painting himself. In an interdependent valuations scenario his valuation for owning the painting would also depend on how much the others like it. Possible reasons are that this influences the painting's potential resale value and the owner's prestige with regard to his standing within the art collector community.

An agent's strategy assigns an action to each of his possible types. In this thesis we focus on direct revelation mechanisms, a special class of mechanisms. In a direct revelation mechanism the only action an agent can take is to announce a type. An agent's strategy is called dominant if it maximizes his utility for every possible combination of actions taken by the other agents. One goal of the mechanism designer could be to design a direct revelation mechanism which is dominant strategy incentive compatible. This means creating a mechanism such that truthfully announcing his own type is a dominant strategy for every agent. In this thesis we are concerned with Bayes-Nash settings. In these settings agents do not know which types the others have but the distribution of potential types for each agent is publicly known. More specifically, we are investigating Bayes-Nash 
incentive compatible direct revelation mechanisms. This means that truthfully reporting his own type maximizes each agent's expected utility, given that all the other agents also report truthfully.

Besides truthfulness a mechanism designer might strive for other goals, for example efficiency. An efficient mechanism picks an outcome that maximizes social welfare. That is, the outcome maximizes the sum total of all agents' valuations. Another potential goal is optimality. In our painting auction example this would mean picking an auction format that maximizes the auctioneer's revenue. In case the agents do not have to participate in the mechanism but can opt out of the whole process the designer might want to consider individual rationality as a design goal in order to assure himself of the agents' participation. Individual rationality ensures that truthfully acting agents receive nonnegative utility when participating in the mechanism.

\subsection{Thesis Outline}

As established before, a mechanism consists of an allocation rule and a payment rule. In a recent stream of literature researchers are concerned with the characterization of dominant strategy incentive compatible direct revelation mechanisms in terms of a monotonicity condition on the allocation alone. That is, they want to characterize precisely those allocation rules for which they can guarantee the existence of a payment rule that makes the resulting mechanism dominant strategy incentive compatible.

In Chapters 2 and 3 we adapt this idea to Bayes-Nash incentive compatible mechanisms. In Chapter 2 we consider direct revelation mechanisms in settings where agents have independently distributed, one-dimensional types and quasi-linear utility functions. ${ }^{1}$ Agents are allowed to have interdependent valuations. We show that monotonicity, a condition on the allocation rule, is a necessary condition for Bayes-Nash incentive compatibility. This condition expresses that an agent's expected gain in valuation for truthfully reporting a type $t$ instead of misreporting a type $s$ should be at least as big as his expected gain in valuation for misreporting $t$ instead of truthfully reporting $s$.

In order to establish the sufficiency of monotonicity for Bayes-Nash incentive compatibility we take a network approach similar to the one taken by Gui, Müller and Vohra (2004) and Saks and Yu (2005) in their work with regard to dominant strategy incentive compatible mechanisms. Recognizing that the constraints inherent in the definition of Bayes-Nash incentive compatibility have a natural network interpretation we build complete directed graphs for agents' type spaces. To do so we

\footnotetext{
${ }^{1}$ This chapter is based on Müller, Perea and Wolf (2005).
} 
associate a node with each type and put a directed edge between each ordered pair of nodes. The length of the edge going from the node associated with type $s$ to the node associated with the type $t$ is defined as the cost of manipulation. That is, the expected difference in an agent's valuation for truthfully reporting $s$ instead of misreporting $t$. Monotonicity corresponds to the absence of 2-cycles with negative length in these graphs.

We show that an allocation rule is Bayes-Nash incentive compatible if and only if the graphs described above contain no negative length cycles. This result is the Bayes-Nash equivalent to a finding by Rochet (1987) who shows that dominant strategy incentive compatibility can be characterized in terms of the absence of negative length cycles in similar graphs.

We demonstrate that in order for monotonicity to be a sufficient condition for Bayes-Nash incentive compatibility we have to put further restrictions on our setting. Such conditions are decomposition monotonicity of the costs of manipulation or agents' valuation functions satisfying non-decreasing expected differences. For these restrictions we can establish the sufficiency of monotonicity. Characterization results of Myerson (1981), Malakhov and Vohra (2004) and Feng (2008) for Bayes-Nash incentive compatible mechanisms follow as special cases from our characterization results. Their settings can be folded into our framework which allows for a broader class of type spaces and alternative forms interdependencies between agents' valuations.

Furthermore, we show how to construct corresponding payment rules for Bayes-Nash incentive compatible allocation rules by using shortest path length in the agent's graphs. An allocation rule satisfies revenue equivalence if all payment rules that make it Bayes-Nash incentive compatible yield the same expected payments up to an additive constant.We establish that revenue equivalence holds if agents' type spaces are convex and their expected valuations are convex in their own types. Thus, we establish revenue equivalence under the same conditions as Krishna and Maenner (2001).

In Chapter 3 we extend the analysis of the foregoing chapter to settings with multi-dimensional type spaces. ${ }^{2}$ Considering the painting auction from the beginning, one component of agent $A$ 's type might reflect how much he likes the painting. Another component could reflect how happy he would be in case agent $B$ wins the painting because they are friends. Yet another component of his type could reflect how sad he would be in case agent $C$ wins because they are long-standing rivals.

We follow the same network approach as before and keep the structure of the chapter as close as possible to the foregoing one. This yields some overlap between both chapters but in this way Chapter 3 can be read as an independent entity. In this chapter we have to restrict agents' type

\footnotetext{
${ }^{2}$ This chapter is based on Müller, Perea and Wolf (2007b).
} 
spaces to be convex and their expected valuation functions to be convex in their own types. We show that if the costs of manipulation are decomposition monotone or agents' valuation functions satisfying non-decreasing expected differences then monotonicity in conjunction with an integrability condition is both necessary and sufficient for Bayes-Nash incentive compatibility. Although monotonicity alone is not sufficient anymore, we still achieve a characterization that is purely based on the valuations and the allocation rule. Characterization results of Jehiel, Moldovanu and Stacchetti (1999), Jehiel and Moldovanu (2001) and Müller, Perea and Wolf (2007b) follow as special cases from our characterization results. Again we can establish revenue equivalence under the same conditions as Krishna and Maenner (2001).

In Chapter 4 we consider scoring auctions. ${ }^{3}$ In the painting auction example described before, competition among the bidders works only via the price. Essentially every bidder announces a price he this willing to pay and whoever makes the highest offer gets the painting. However, sometimes the price of an item is not the only concern and other non-monetary attributes play a role as well. Consider for example several construction companies competing for a government contract to build a highway. One important feature is of course the price. Another one is the completion time. So one company might offer to build the highway for 20 million dollars within 6 months and another company might offer to build it for 15 million dollars but only within 9 months.

One common method employed to deal with the analysis of scoring auction settings is to skillfully transform the setting into that of a standard, price-only auction. Then known results about these price-only auctions can be applied. We do this for a scoring auction setting in which several items with multiple attributes need to be allocated. We show how to transform this setting into a combinatorial auction setting that looks like the linear valuations setting discussed in Section 3.4.1 of the foregoing chapter. In doing this we extend the work of Asker and Cantillon (2008) to a framework with multiple items. Asker and Cantillon (2008) consider a scoring auction setting in which a single item with multiple attributes has to be allocated. They transform this into a single item, price-only auction setting.

\footnotetext{
${ }^{3}$ This chapter is based on Müller, Perea and Wolf (2007a).
} 



\section{Monotonicity and One-Dimensional Types}

An allocation rule is called Bayes-Nash incentive compatible, if there exists a payment rule, such that truthful reports of agents' types form a Bayes-Nash equilibrium in the direct revelation mechanism consisting of the allocation rule and the payment rule. This chapter provides characterizations of Bayes-Nash incentive compatible allocation rules in social choice settings where agents have onedimensional and independently distributed types, quasi-linear utility functions and interdependent valuations. The characterizations are derived by constructing complete directed graphs on agents' type spaces with cost of manipulation as lengths of edges. Thus, the work presented in this chapter serves as the Bayes-Nash extension of a recent stream of literature that employs a network based approach to characterize dominant strategy incentive compatible allocation rules.

Monotonicity of the allocation rule corresponds to the condition that all 2-cycles in the above mentioned graphs have non-negative length. In case agents' valuation functions satisfy non-decreasing expected differences, it is shown that monotonicity is a necessary and sufficient condition for BayesNash incentive compatibility. The characterization of Bayes-Nash incentive compatibility in models with one-dimensional types is a well-explored field. Next to a condition on the allocation rule such characterizations commonly employ also conditions on agents' utility functions. Here we provide a characterization in terms of a simple property on the allocation alone. Our approach also allows us to work in a very general setup that allows for broad forms of interdependent valuations and finite as well as infinite type spaces.

It is shown how corresponding payment rules are constructed for Bayes-Nash incentive compatible allocation rules by utilizing shortest path lengths in agents' graphs. In the case that agents have convex type spaces and expected valuations which are convex in their types, it is established that revenue equivalence holds for all incentive compatible allocation rules. This result was already established by Krishna and Maenner (2001). However, our network approach yields an alternate proof of this result. 


\section{$2.1 \quad$ Introduction}

In this chapter we concern ourselves with social choice settings where agents have independently distributed, one-dimensional types and quasi-linear utility functions. That is, an agent's utility is his valuation of an outcome minus a payment. We allow for interdependent valuations across agents. Thus, an agent's valuation of an outcome can depend on the types of other agents as well. We consider direct revelation mechanisms. Direct mechanisms consist of two rules: an allocation rule and a payment rule. Given a profile of type reports from all agents, the allocation rule determines the outcome, for example who wins the object in a single item auction. The payment rule determines the payment each agent has to make. An allocation rule is called Bayes-Nash incentive compatible if there exists a payment rule such that truthful reporting of agents' types constitutes a Bayes-Nash equilibrium in the corresponding direct revelation mechanism consisting of the two rules.

One major issue addressed in this chapter is the characterization of Bayes-Nash incentive compatible allocation rules in the social choice settings under consideration. That is, we want to characterize precisely those allocation rules for which we can guarantee the existence of a payment rule making them Bayes-Nash incentive compatible. Additionally, we aim for a framework that enables us to construct such a payment rule for a particular allocation rule, if one exists. Take for example an allocation rule deciding in a single item auction for each set of bids which agent wins the object. We want to be able to decide whether there exists a pricing scheme for winning bids that makes truthful bidding a Bayes-Nash equilibrium. In case the answer is yes, we want to have the means to construct such a pricing scheme.

The construction of payment rules is the second major issue addressed in this chapter. We show how to build a payment rule that makes an allocation rule Bayes-Nash incentive compatible. Furthermore, we investigate how much freedom exists in the construction process. Do all payment rules that make a particular allocation rule Bayes-Nash incentive compatible yield the same expected payments for the agents? Or is there room for the existence of payment rules that yield differing expected payments for the agents?

\subsubsection{Related Work}

A recent stream of literature has focused on the characterization of dominant strategy incentive compatible allocation rules in terms of a monotonicity condition on the allocation rule. An allocation rule is dominant strategy incentive compatible, if there exists a payment rule such that for any report of the other agents an agent maximizes his own utility by reporting truthfully his type. Examples 
are Bikhchandani, Chatterji, Lavi, Mu'alem, Nisan and Sen (2006), Monderer (2008), Gui, Müller and Vohra (2004) and Saks and Yu (2005).

The later two establish their results by making a link to network theory. Take for example Saks and $\mathrm{Yu}$ (2005). They consider a setting in which the allocation rule maps agents' types into a finite set of possible outcomes. It is shown that dominant strategy incentive compatible allocation rules in this setting can be characterized in terms of weak monotonicity. In order to derive this result they construct complete directed graphs in the following way: Take some agent and fix a profile of type reports for the others. Now, a directed graph is constructed by associating a node with each outcome and putting a directed edge between each ordered pair of nodes. Take two outcomes $a$ and $b$. Consider the difference of the valuation of $a$ and the valuation of $b$ with respect to every type for which truthfully reporting this type yields outcome $a$. The length of the network edge from $a$ to $b$ is defined as the infimum of all these differences. In this fashion a graph is constructed for every agent and every possible report profile of the other agents. Weak monotonicity states that for any two different outcomes $a$ and $b$, the sum of the two edge lengths from $a$ to $b$ and from $b$ to $a$ is non-negative.

Earlier, Rochet (1987) characterized dominant strategy implementation in cases where the set of outcomes is not necessarily finite, an assumption that is made in all of the above mentioned references. For the case where agents have one-dimensional, convex type spaces and their valuation functions satisfy the increasing differences property, Rochet(1987) shows that dominant strategy incentive compatibility can be characterized in terms of a monotonicity condition on the allocation rule alone.

Monotonicity in conjunction with a condition on agents' utility functions is also used to characterize Bayes-Nash incentive compatible allocation rules in settings featuring one-dimensional types, see for example Mas-Collel, Whinston and Green (1995), Chapter 23, or Jehle and Reny (2001), Chapter 9. Myerson (1981) gives such a characterization for a single item auction setting where agents have one-dimensional, convex type spaces and interdependent valuations. A similar characterization result is provided by Feng (2008). In her setting multiple, commonly ranked items are auctioned off to agents having one-dimensional, convex type spaces and independent valuations.

Malakhov and Vohra (2004) consider an auction setting where multiple units of an homogeneous good are auctioned off to several agents with constant marginal valuations. The one-dimensional type of an agent represents his marginal valuation for an extra unit of the good. Each agent has a finite set of possible types. Agents' valuations are independent. Malakhov and Vohra (2004) show 
that Bayes-Nash incentive compatibility holds if and only if the allocation rule is monotone. So their characterization is in terms of a monotonicity condition on the allocation rule alone. In order to establish this result they also make a link to network theory.

Archer and Kleinberg (2008) consider a single agent setting featuring linear valuation functions. For convex type spaces they characterize incentive compatibility in terms of a monotonicity and a local integrability condition of the allocation rule. They also deliver some insight for non-convex type spaces.

In addition to the characterization result mentioned above, Myerson (1981) also shows that in the setting considered by him revenue equivalence holds for all Bayes-Nash incentive compatible allocation rules. Krishna and Maenner (2001) provide such a result for a setting where each agent has a convex type space and a valuation function that is convex in the agent's type. Heydenreich, Müller, Uetz and Vohra (2009) derive a characterization of revenue equivalence under more general conditions. Similar to Gui, Müller and Vohra (2004) and Saks and Yu (2005) they use networks to establish their results. For countable outcome spaces the aforementioned result of Krishna and Maenner (2001) follows as a consequence of their work.

\subsubsection{Our Contribution}

In Section 2.2 we state some basic assumptions and definitions. Throughout the chapter we assume that agents have quasi-linear utility functions and independently distributed, privately known, one-dimensional types. Furthermore, we allow for interdependent valuations. We do not put any restrictions on the number of possible outcomes.

If an allocation rule is Bayes-Nash incentive compatible, then there exists a payment rule such that an agent's expected utility for truthfully reporting his type $t$ is at least as high as his expected utility for misreporting some type $s$. Similarly, an agent's expected utility for truthfully reporting type $s$ is at least as high as his expected utility for misreporting type $t$. By combining these two conditions we get a monotonicity condition on the allocation rule. This condition is the expected utility equivalent to the weak monotonicity condition utilized by Saks and Yu (2005) in the context of dominant strategy incentive compatible allocation rules. Monotonicity is a necessary condition for Bayes-Nash incentive compatibility. It expresses that the expected gain in valuation for truthfully reporting $t$ instead of misreporting $s$ should be at least as big as the expected gain in valuation for misreporting $t$ instead of truthfully reporting $s$.

Similar to the network approach of Gui, Müller and Vohra (2004) and Saks and Yu (2005) we 
construct graphs in Section 2.3. Recognizing that the constraints inherent in the definition of BayesNash incentive compatibility have a natural network interpretation we build complete directed graphs for agents' type spaces. To do so we associate a node with each type and put a directed edge between each ordered pair of nodes. The length of the edge going from the node associated with type $s$ to the node associated with the type $t$ is defined as the cost of manipulation. That is, the expected difference in an agent's valuation for truthfully reporting $s$ instead of misreporting $t$.

Different from the networks constructed by Gui, Müller and Vohra (2004) and Saks and Yu (2005) (see description earlier in this section) we construct only one graph for each agent. This is because we work in terms of expectations and do not consider each possible type profile of the other agents separately. Furthermore, each of these graphs contains (except for the case of finite type spaces) an infinite number of nodes as we associate a node with each possible type of an agent. One could also construct outcome based graphs, as done by Gui, Müller and Vohra (2004) and Saks and Yu (2005), by associating a node with each possible probability distribution over outcomes. However, these graphs possibly also contain an infinite number of nodes since we allow that the different possible type reports of an agent induce an infinite number of probability distributions over outcomes. For example, in the single item auction framework presented in Section 2.6.1 it is quite natural to allow that every different type report of an agent yields a different expected conditional probability for him to win the object.

We show that an allocation rule is Bayes-Nash incentive compatible if and only if the networks described above contain no negative length cycles. Rochet (1987) shows that dominant strategy incentive compatibility can be characterized in terms of the absence of negative length cycles in similar graphs. Our result is the Bayes-Nash equivalent for his finding. Furthermore, we show as a lemma that the costs of manipulation are decomposition monotone if the allocation rule satisfies monotonicity and agents' valuation functions satisfy non-decreasing expected differences (definitions can be found in Section 2.2).

Agents' types are restricted to be one-dimensional. No further restrictions on agents' type spaces are made. That is, we allow for infinite type spaces as considered by Myerson (1981) and Feng (2008) as well as for finite type spaces as considered by Malakhov and Vohra (2004). Without any additional restrictions our setting is too general in order for monotonicity to be a sufficient condition for Bayes-Nash incentive compatibility. This is illustrated by an example in Section 2.4. However, for the case that the costs of manipulation are assumed to be decomposition monotone we are able to show that monotonicity is a necessary and sufficient condition for Bayes-Nash incentive compatibility. 
Using the lemma from Section 2.3 it follows that monotonicity is a necessary and sufficient condition for Bayes-Nash incentive compatibility for the case that agents' valuation functions satisfy nondecreasing expected differences. The aforementioned auction settings of Malakhov and Vohra (2004), Myerson (1981) and Feng (2008) are special cases of this framework. Compared to their settings, our framework allows not only for a broader class of type spaces but also for alternative forms of interdependencies between agents' valuations. How their settings can be folded into our framework is illustrated at the end of this chapter in Section 2.6.

Thus, the main contribution of Section 2.4 is to derive a complete characterization of Bayes-Nash incentive compatibility in terms of monotonicity. Thereby we achieve a characterization that depends purely on the valuations and the allocation rule. This is done in a very broad framework that allows for finite and infinite type spaces as well as interdependent valuations. Our characterization resembles the one derived by Rochet (1987) for dominant strategy incentive compatibility. However, our results do not follow from Rochet immediately. Rochet (1987) requires agents to have convex type spaces and twice differentiable valuation functions, whereas our setting allows for broader classes of type spaces and valuation functions.

If we know that an allocation rule is Bayes-Nash incentive compatible, we would also like to know how to construct a corresponding payment rule. In Section 2.5 we show that such payment rules can be built by making use of shortest path lengths in the agents' networks. We also investigate how much room there exists for having different payment rules that make a particular allocation rule BayesNash incentive compatible. Specifically, we establish that Bayes-Nash incentive compatible allocation rules satisfy revenue equivalence if agents have convex type spaces and expected valuations that are convex in their own types. Thus, using our network approach, we can establish revenue equivalence under the same conditions as Krishna and Maenner (2001). Using examples featuring discrete type spaces and concave valuation functions we illustrate that these conditions cannot easily be relaxed.

\subsection{Model \& Definitions}

We consider a setting in which there is a set of possible outcomes $\Gamma$, as well as a set of agents $N=\{1, \ldots, n\}$. Each agent has some private information which is modeled by a one-dimensional type. Specifically, each agent $i$ has a type $t^{i} \in T^{i}$ with $T^{i} \subseteq \mathbb{R}$. The set of all complete type profiles $t=\left(t^{1}, \ldots, t^{n}\right)$ is denoted $T$, whereas the set of all type profiles excluding agent $i, t^{-i}=$ $\left(t^{1}, \ldots, t^{i-1}, t^{i+1}, \ldots, t^{n}\right)$, is denoted $T^{-i}$. We assume that agents' types are independently distributed. Assuming that agent $i$ 's types are distributed according to some density $\pi^{i}$ on $T^{i}$, the joint 
density $\pi^{-i}$ on $T^{-i}$ is then given by

$$
\pi^{-i}\left(t^{-i}\right)=\prod_{\substack{j \in N \\ j \neq i}} \pi^{j}\left(t^{j}\right)
$$

We consider direct revelation mechanisms, consisting of a payment rule and an allocation rule. Based on a profile of reported types from all the agents, a payment rule

$$
P: T \mapsto \mathbb{R}^{n}
$$

assigns each agent with a payment. So, given a report profile $r^{-i}$ of the others, reporting type $r^{i}$ results in a payment $P_{i}\left(r^{i}, r^{-i}\right)$ for agent $i$. Similarly, an allocation rule

$$
f: T \mapsto \Gamma
$$

maps each profile of reported types into an outcome.

Suppose agent $i$ has true type $t^{i}$ and reports $r^{i}$ while the others have true types $t^{-i}$ and report $r^{-i}$. The value that agent $i$ assigns to the resulting outcome of the allocation rule is denoted by $v^{i}\left(f\left(r^{i}, r^{-i}\right) \mid t^{i}, t^{-i}\right)$. Thus, we allow for interdependent valuations across agents. That is, an agent's valuation for an outcome is not only influenced by his own type but also by the types of the other agents. Take for example an auction for a painting, see Klemperer (1999), where agents' types reflect how much they like the painting. An agent's valuation for owning the painting depends on the types of the others as they affect the possible resale value of the painting and the owner's prestige.

Utilities are quasi-linear. That is, an agent's utility is his valuation of an allocation minus his payment. Let us consider agent $i$ and assume that all other agents report truthfully. If agent $i$ has true type $t^{i}$, then his expected utility for making a report $r^{i}$ is given by

$$
\begin{aligned}
U^{i}\left(r^{i} \mid t^{i}\right) & =\int_{T^{-i}}\left(v^{i}\left(f\left(r^{i}, t^{-i}\right) \mid t^{i}, t^{-i}\right)-P_{i}\left(r^{i}, t^{-i}\right)\right) \pi^{-i}\left(t^{-i}\right) d t^{-i} \\
& =E_{-i}\left[v^{i}\left(f\left(r^{i}, t^{-i}\right) \mid t^{i}, t^{-i}\right)-P_{i}\left(r^{i}, t^{-i}\right)\right] .
\end{aligned}
$$

If agent $i$ 's types have a discrete distribution this simplifies to

$$
\begin{aligned}
U^{i}\left(r^{i} \mid t^{i}\right) & =\sum_{t^{-i} \in T^{-i}}\left(v^{i}\left(f\left(r^{i}, t^{-i}\right) \mid t^{i}, t^{-i}\right)-P_{i}\left(r^{i}, t^{-i}\right)\right) \pi^{-i}\left(t^{-i}\right) \\
& =E_{-i}\left[v^{i}\left(f\left(r^{i}, t^{-i}\right) \mid t^{i}, t^{-i}\right)-P_{i}\left(r^{i}, t^{-i}\right)\right] .
\end{aligned}
$$


We assume $E_{-i}\left[v^{i}\left(f\left(r^{i}, t^{-i}\right) \mid t^{i}, t^{-i}\right)\right]$ to be finite $\forall r^{i}, t^{i} \in T^{i}$. This holds for example if $\forall r^{i}, t^{i}$ the valuation function $v^{i}\left(f\left(r^{i}, t^{-i}\right) \mid t^{i}, t^{-i}\right)$, viewed as a function of $t^{-i}$, is bounded. This also holds in the discrete case.

Definition 2.1 (Bayes-Nash Incentive Compatibility) An allocation rule $f$ is Bayes-Nash incentive compatible if there exists a payment rule $P$ such that $\forall i \in N$ and $\forall r^{i}, \tilde{r}^{i} \in T^{i}$ :

$$
E_{-i}\left[v^{i}\left(f\left(r^{i}, t^{-i}\right) \mid r^{i}, t^{-i}\right)-P_{i}\left(r^{i}, t^{-i}\right)\right] \geq E_{-i}\left[v^{i}\left(f\left(\tilde{r}^{i}, t^{-i}\right) \mid r^{i}, t^{-i}\right)-P_{i}\left(\tilde{r}^{i}, t^{-i}\right)\right]
$$

If an allocation rule $f$ is Bayes-Nash incentive compatible, we have also

$$
E_{-i}\left[v^{i}\left(f\left(\tilde{r}^{i}, t^{-i}\right) \mid \tilde{r}^{i}, t^{-i}\right)-P_{i}\left(\tilde{r}^{i}, t^{-i}\right)\right] \geq E_{-i}\left[v^{i}\left(f\left(r^{i}, t^{-i}\right) \mid \tilde{r}^{i}, t^{-i}\right)-P_{i}\left(r^{i}, t^{-i}\right)\right]
$$

By adding (2.3) and (2.4) we get

$$
\begin{aligned}
& E_{-i}\left[v^{i}\left(f\left(r^{i}, t^{-i}\right) \mid r^{i}, t^{-i}\right)+v^{i}\left(f\left(\tilde{r}^{i}, t^{-i}\right) \mid \tilde{r}^{i}, t^{-i}\right)-P_{i}\left(r^{i}, t^{-i}\right)-P_{i}\left(\tilde{r}^{i}, t^{-i}\right)\right] \\
\geq & E_{-i}\left[v^{i}\left(f\left(\tilde{r}^{i}, t^{-i}\right) \mid r^{i}, t^{-i}\right)+v^{i}\left(f\left(r^{i}, t^{-i}\right) \mid \tilde{r}^{i}, t^{-i}\right)-P_{i}\left(r^{i}, t^{-i}\right)-P_{i}\left(\tilde{r}^{i}, t^{-i}\right)\right] .
\end{aligned}
$$

Notice that agent $i$ 's expected payments for reporting types $r^{i}$ and $\tilde{r}^{i}$ appear on both sides of the above inequality and can be canceled. This however is only possible because we work under the assumption of independently distributed types. Dropping this assumption would result in the following: Because of stemming from (2.3), the expected payment for reporting $r^{i}$ on the left-hand side of (2.5) would be conditional on agent $i$ having true type $r^{i}$. On the other hand, the expected payment for reporting $r^{i}$ on the right-hand side of (2.5) would be conditional on agent $i$ having true type $\tilde{r}^{i}$ since it stems from (2.4). Thus, the two expected payments could not be canceled anymore.

Canceling the expected payments and rearranging the terms in (2.5) yields the following monotonicity condition:

Definition 2.2 (Monotonicity) An allocation rule $f$ satisfies monotonicity if $\forall i \in N$ and $\forall r^{i}, \tilde{r}^{i} \in$ $T^{i}$ :

$$
\begin{aligned}
& E_{-i}\left[v^{i}\left(f\left(r^{i}, t^{-i}\right) \mid r^{i}, t^{-i}\right)-v^{i}\left(f\left(\tilde{r}^{i}, t^{-i}\right) \mid r^{i}, t^{-i}\right)\right] \\
\geq & E_{-i}\left[v^{i}\left(f\left(r^{i}, t^{-i}\right) \mid \tilde{r}^{i}, t^{-i}\right)-v^{i}\left(f\left(\tilde{r}^{i}, t^{-i}\right) \mid \tilde{r}^{i}, t^{-i}\right)\right] .
\end{aligned}
$$


The above condition is the expected utility equivalent to the 2-cycle inequality of Gui, Müller and Vohra (2004), the weak monotonicity (W-Mon) condition of Bikhchandani, Chatterji, Lavi, Mu'alem, Nisan and Sen (2006) and the weak monotonicity condition of Saks and Yu (2005). It is the same as the condition termed weak monotonicity in Müller, Perea and Wolf (2007b). The rationale for naming this condition monotonicity becomes evident once we consider valuation functions that are linear with respect to agents' types, see for example the applications presented in Section 2.6 of this chapter. Obviously, monotonicity is a necessary condition for Bayes-Nash incentive compatibility. In Section 2.4 we discuss the sufficiency of monotonicity.

Furthermore, let us introduce at this point the following condition for agents' valuation functions:

Definition 2.3 (Non-decreasing Expected Differences) Consider $r^{i}, \tilde{r}^{i}, t^{i}, \tilde{t}^{i} \in T^{i}$ such that

$$
\begin{aligned}
& E_{-i}\left[v^{i}\left(f\left(r^{i}, t^{-i}\right) \mid t^{i}, t^{-i}\right)-v^{i}\left(f\left(\tilde{r}^{i}, t^{-i}\right) \mid t^{i}, t^{-i}\right)\right] \\
\geq & E_{-i}\left[v^{i}\left(f\left(r^{i}, t^{-i}\right) \mid \tilde{t}^{i}, t^{-i}\right)-v^{i}\left(f\left(\tilde{r}^{i}, t^{-i}\right) \mid \tilde{t}^{i}, t^{-i}\right)\right] .
\end{aligned}
$$

The valuation function satisfies non-decreasing expected differences if $\forall \bar{t}^{i} \in T^{i}$ s.t. $\bar{t}^{i}=(1-\alpha) \tilde{t}^{i}+\alpha t^{i}, \alpha>1$ we have

$$
\begin{aligned}
& E_{-i}\left[v^{i}\left(f\left(r^{i}, t^{-i}\right) \mid \bar{t}^{i}, t^{-i}\right)-v^{i}\left(f\left(\tilde{r}^{i}, t^{-i}\right) \mid \bar{t}^{i}, t^{-i}\right)\right] \\
\geq & E_{-i}\left[v^{i}\left(f\left(r^{i}, t^{-i}\right) \mid t^{i}, t^{-i}\right)-v^{i}\left(f\left(\tilde{r}^{i}, t^{-i}\right) \mid t^{i}, t^{-i}\right)\right] .
\end{aligned}
$$

The above condition deals with the marginal change in expected valuation with respect to the reported type. It is illustrated in Figure 2.1. Consider the change in expected valuation for making a report $r^{i}$ instead of $\tilde{r}^{i}$. Assume that there exist types $t^{i}$ and $\tilde{t}^{i}$ such that this change is larger or at least as large if the agent has true type $t^{i}$ instead of $\tilde{t}^{i}$. Now consider the agent having a true type which is even further away from $\tilde{t}^{i}$ than $t^{i}$ (in the direction of $t^{i}$ ). The condition then requires that the change in expected valuation is at least as large as in the case where the agent has true type $t^{i}$. That is, the point corresponding to this change in expected valuation in Figure 2.1 has to lie in the shaded area. This requirement is slightly weaker than the condition known as increasing (or isotone) differences (see for example Milgrom (2004)) which asserts that the marginal change in valuation with respect to the allocation is strictly increasing in the type. Specifically, the increasing differences condition 


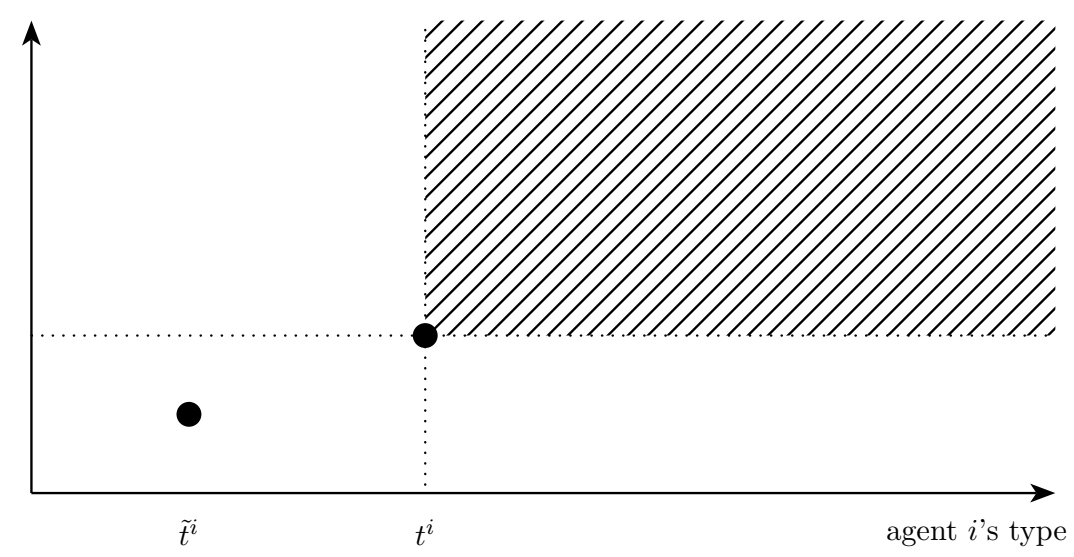

Figure 2.1: Illustration non-decreasing expected differences: The change in expected valuation for making a report $r^{i}$ instead of $\tilde{r}^{i}, E_{-i}\left[v^{i}\left(f\left(r^{i}, t^{-i}\right) \mid ., t^{-i}\right)-v^{i}\left(f\left(\tilde{r}^{i}, t^{-i}\right) \mid ., t^{-i}\right)\right]$, is depicted along the $y$-axis.

requires that for all $r^{i}>\hat{r}^{i}$ and all $t^{i}>\hat{t}^{i}$ in $T^{i}$,

$$
\begin{aligned}
& E_{-i}\left[v^{i}\left(f\left(r^{i}, t^{-i}\right) \mid t^{i}, t^{-i}\right)-v^{i}\left(f\left(\hat{r}^{i}, t^{-i}\right) \mid t^{i}, t^{-i}\right)\right] \\
> & E_{-i}\left[v^{i}\left(f\left(r^{i}, t^{-i}\right) \mid \hat{t}^{i}, t^{-i}\right)-v^{i}\left(f\left(\hat{r}^{i}, t^{-i}\right) \mid \hat{t}^{i}, t^{-i}\right)\right] .
\end{aligned}
$$

Note that an agent's valuation function which is linear in his true type satisfies the non-decreasing expected differences condition, see for example the application settings discussed in Section 2.6.

\subsection{A Network Interpretation}

We begin this section by briefly reviewing a well-known result from the field of network flow theory. ${ }^{1}$ Let $X=\left\{x_{1}, \ldots, x_{k}\right\}$ be a finite set of variables. Consider the following system of constraints:

$$
x_{i}-x_{j} \leq w_{i j} \quad \forall i, j \in\{1, \ldots, k\},
$$

where $w_{i j}$ is some constant specific to the ordered pair $(i, j)$. The system can be associated with a network by constructing a directed, weighted graph whose nodes correspond to the variables. A directed edge is put between each ordered pair of nodes. The length of the edge from the node

\footnotetext{
${ }^{1}$ For a comprehensive introduction to network flows we refer the reader to Ahuja, Magnanti and Orlin (1993).
} 
corresponding to $x_{i}$ to the node corresponding to $x_{j}$ is given by $w_{i j}$.

It is a well-known result (see for example Shostak (1981)) that there is no negative length cycle in the associated network if and only if the system of linear inequalities in (2.6) is feasible. The system being feasible means that there exists an assignment of real values to the variables such that the constraints in (2.6) are satisfied. Furthermore, if the system is feasible then one feasible solution is to assign to each $x_{i}$ the length of a shortest path from the node associated with $x_{i}$ to some arbitrary source node. ${ }^{2}$

In order to see that the constraints in (2.3) have a natural network interpretation it is useful to rewrite (2.3) as follows:

$$
E_{-i}\left[P_{i}\left(r^{i}, t^{-i}\right)-P_{i}\left(\tilde{r}^{i}, t^{-i}\right)\right] \leq E_{-i}\left[v^{i}\left(f\left(r^{i}, t^{-i}\right) \mid r^{i}, t^{-i}\right)-v^{i}\left(f\left(\tilde{r}^{i}, t^{-i}\right) \mid r^{i}, t^{-i}\right)\right] .
$$

Considering a specific allocation rule, the right-hand side of (2.7) is a constant. Thus, we have a system of difference constraints as described in (2.6) (except that we are now dealing with a potentially infinite number of variables and constraints).

Given this observation, we associate the system of inequalities (2.7) with a network in the same way as is described above. For each agent we build a complete directed graph $T_{f}^{i}$. A node is associated with each type and a directed edge is put between each ordered pair of nodes. For agent $i$ the length of an edge directed from $r^{i}$ to $\tilde{r}^{i}$ is denoted $l^{i}\left(r^{i}, \tilde{r}^{i}\right)$ and is defined as the cost of manipulation:

$$
l^{i}\left(r^{i}, \tilde{r}^{i}\right)=E_{-i}\left[v^{i}\left(f\left(r^{i}, t^{-i}\right) \mid r^{i}, t^{-i}\right)-v^{i}\left(f\left(\tilde{r}^{i}, t^{-i}\right) \mid r^{i}, t^{-i}\right)\right]
$$

Given our previous assumptions, the edge length is finite. For technical reasons we allow for loops. However, note that an edge directed from $r^{i}$ to $r^{i}$ has length $l^{i}\left(r^{i}, r^{i}\right)=0$.

Using this definition of the edge lengths, the monotonicity condition can be written as

$$
l^{i}\left(r^{i}, \tilde{r}^{i}\right)+l^{i}\left(\tilde{r}^{i}, r^{i}\right) \geq 0 \quad \forall i \in N, \forall r^{i}, \tilde{r}^{i} \in T^{i} .
$$

So monotonicity corresponds to the absence of negative length 2-cycles in the agents' graphs described above.

Rochet (1987) observed that dominant strategy incentive compatibility can be characterized in

\footnotetext{
${ }^{2}$ In order to be consistent with the existing literature we defined the system of constraints as in (2.6). However, in network theory the constraints are commonly defined as $x_{j}-x_{i} \leq w_{i j}$. In this case, if the system is feasible then one feasible solution is to assign to each $x_{i}$ the length of a shortest path from some arbitrary source node to the node associated with $x_{i}$.
} 
terms of the absence of negative length cycles in similar graphs. Using the same proof technique, we can derive such a characterization for Bayes-Nash incentive compatibility as well.

Theorem 2.1 An allocation rule $f$ is Bayes-Nash incentive compatible if and only if there is no negative length cycle in $T_{f}^{i}, \forall i \in N$.

Proof (Adapted from Rochet (1987).)

Take some agent $i$ and let $C=\left(r_{1}^{i}, \ldots, r_{m}^{i}, r_{m+1}^{i}=r_{1}^{i}\right)$ denote a cycle in $T_{f}^{i}$. Let us assume that $f$ is Bayes-Nash incentive compatible. Using (2.7) and the edge length definition (2.8), this implies that for every $j \in\{1, \ldots, m\}$,

$$
E_{-i}\left[P_{i}\left(r_{j}^{i}, t^{-i}\right)-P_{i}\left(r_{j+1}^{i}, t^{-i}\right)\right] \leq l^{i}\left(r_{j}^{i}, r_{j+1}^{i}\right)
$$

Adding up these inequalities yields

$$
0 \leq \sum_{j=1}^{m} l^{i}\left(r_{j}^{i}, r_{j+1}^{i}\right),
$$

so cycle $C$ has non-negative length.

Conversely, let us assume that there exists no negative length cycle in $T_{f}^{i}, \forall i \in N$. For each agent $i$ we pick an arbitrary source node $r_{0}^{i} \in T^{i}$ and define $\forall r^{i} \in T^{i}$

$$
p^{i}\left(r^{i}\right)=\inf \sum_{j=1}^{m} l^{i}\left(r_{j}^{i}, r_{j+1}^{i}\right),
$$

where the infimum is taken over all paths $A=\left(r_{1}^{i}=r^{i}, \ldots, r_{m+1}^{i}=r_{0}^{i}\right)$ in $T_{f}^{i}$, that is, all paths that start at $r^{i}$ and end at $r_{0}^{i}$. Absence of negative length cycles implies that $p^{i}\left(r_{0}^{i}\right)=0$. Furthermore, $\forall r^{i} \in T^{i}$ we have

$$
p^{i}\left(r_{0}^{i}\right) \leq p^{i}\left(r^{i}\right)+l^{i}\left(r_{0}^{i}, r^{i}\right)
$$

which implies that $p^{i}\left(r^{i}\right)$ is finite. For every pair $r^{i}, \tilde{r}^{i} \in T^{i}$ we also have

$$
p^{i}\left(r^{i}\right) \leq p^{i}\left(\tilde{r}^{i}\right)+l^{i}\left(r^{i}, \tilde{r}^{i}\right)
$$

Thus, by setting

$$
P_{i}\left(r^{i}, t^{-i}\right)=p^{i}\left(r^{i}\right) \quad \forall t^{-i} \in T^{-i}
$$


and using (2.8) we get

$$
E_{-i}\left[P_{i}\left(r^{i}, t^{-i}\right)-P_{i}\left(\tilde{r}^{i}, t^{-i}\right)\right] \leq E_{-i}\left[v^{i}\left(f\left(r^{i}, t^{-i}\right) \mid r^{i}, t^{-i}\right)-v^{i}\left(f\left(\tilde{r}^{i}, t^{-i}\right) \mid r^{i}, t^{-i}\right)\right] .
$$

Hence, the constraints in (2.7) are satisfied and $f$ is Bayes-Nash incentive compatible. Note that it is actually sufficient to pick a payment rule such that $E_{-i}\left[P_{i}\left(r^{i}, t^{-i}\right)\right]=p^{i}\left(r^{i}\right)+c^{i}$. This allows for a variety of payment rules yielding the same expected payments up to an additive constant.

Let us conclude this section with a condition for the costs of manipulation that is used in the derivation of the characterization theorems presented in the following section.

Definition 2.4 (Decomposition Monotonicity) The costs of manipulation are decomposition monotone if $\forall \underline{r}^{i}, \bar{r}^{i} \in T^{i}$ and $\forall r^{i} \in T^{i}$ s.t. $r^{i}=(1-\alpha) \underline{r}^{i}+\alpha \bar{r}^{i}, \alpha \in(0,1)$ we have

$$
l^{i}\left(\underline{r}^{i}, \bar{r}^{i}\right) \geq l^{i}\left(\underline{r}^{i}, r^{i}\right)+l^{i}\left(r^{i}, \bar{r}^{i}\right) .
$$

So considering a pair of nodes, if decomposition monotonicity holds then the direct edge between those nodes is at least as long as any path connecting the same two nodes via nodes lying on the line segment between them. An illustrative example is given in Figure 2.2. Decomposition monotonicity implies that the edge from $\underline{r}^{i}$ to $\bar{r}^{i}$ is at least as long as the path $A=\left(\underline{r}^{i}, r_{* *}^{i}, \bar{r}^{i}\right)$ and that $A$ is at least as long as the path $\tilde{A}=\left(\underline{r}^{i}, r_{*}^{i}, r_{* *}^{i}, r_{* * *}^{i}, \bar{r}^{i}\right)$.

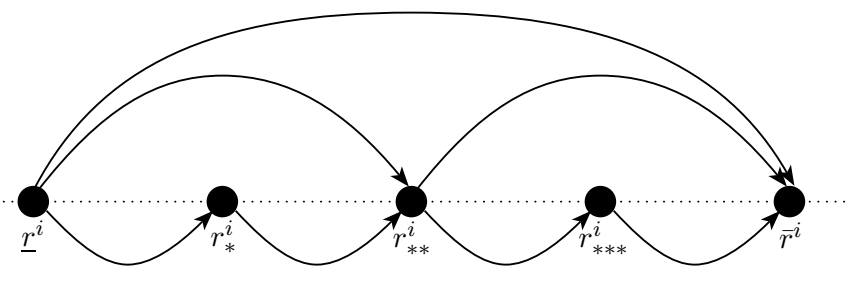

Figure 2.2: Example decomposition monotonicity.

Monotonicity and non-decreasing expected differences together imply that the costs of manipulation are decomposition monotone:

Lemma 2.1 If $f$ satisfies monotonicity and the valuation function satisfies non-decreasing expected differences then the costs of manipulation are decomposition monotone. 


\section{Proof}

Take some agent $i$ and let $\underline{r}^{i}, \bar{r}^{i} \in T^{i}$. Let $r^{i} \in T^{i}$ such that $r^{i}=(1-\alpha) \underline{r}^{i}+\alpha \bar{r}^{i}$ for some $\alpha \in(0,1)$.

Monotonicity implies that

$$
\begin{aligned}
& E_{-i}\left[v^{i}\left(f\left(r^{i}, t^{-i}\right) \mid r^{i}, t^{-i}\right)-v^{i}\left(f\left(\bar{r}^{i}, t^{-i}\right) \mid r^{i}, t^{-i}\right)\right] \\
\geq & E_{-i}\left[v^{i}\left(f\left(r^{i}, t^{-i}\right) \mid \bar{r}^{i}, t^{-i}\right)-v^{i}\left(f\left(\bar{r}^{i}, t^{-i}\right) \mid \bar{r}^{i}, t^{-i}\right)\right] .
\end{aligned}
$$

Since the valuation function satisfies non-decreasing expected differences we have

$$
\begin{aligned}
& E_{-i}\left[v^{i}\left(f\left(r^{i}, t^{-i}\right) \mid \underline{r}^{i}, t^{-i}\right)-v^{i}\left(f\left(\bar{r}^{i}, t^{-i}\right) \mid \underline{r}^{i}, t^{-i}\right)\right] \\
\geq & E_{-i}\left[v^{i}\left(f\left(r^{i}, t^{-i}\right) \mid r^{i}, t^{-i}\right)-v^{i}\left(f\left(\bar{r}^{i}, t^{-i}\right) \mid r^{i}, t^{-i}\right)\right] .
\end{aligned}
$$

Adding $E_{-i}\left[v^{i}\left(f\left(\underline{r}^{i}, t^{-i}\right) \mid \underline{r}^{i}, t^{-i}\right)-v^{i}\left(f\left(r^{i}, t^{-i}\right) \mid \underline{r}^{i}, t^{-i}\right)\right]$ on both sides of the later inequality yields

$$
\begin{aligned}
& E_{-i}\left[v^{i}\left(f\left(r^{i}, t^{-i}\right) \mid \underline{r}^{i}, t^{-i}\right)-v^{i}\left(f\left(\bar{r}^{i}, t^{-i}\right) \mid \underline{r}^{i}, t^{-i}\right)\right] \\
& +E_{-i}\left[v^{i}\left(f\left(\underline{r}^{i}, t^{-i}\right) \mid \underline{r}^{i}, t^{-i}\right)-v^{i}\left(f\left(r^{i}, t^{-i}\right) \mid \underline{r}^{i}, t^{-i}\right)\right] \\
\geq & E_{-i}\left[v^{i}\left(f\left(r^{i}, t^{-i}\right) \mid r^{i}, t^{-i}\right)-v^{i}\left(f\left(\bar{r}^{i}, t^{-i}\right) \mid r^{i}, t^{-i}\right)\right] \\
& +E_{-i}\left[v^{i}\left(f\left(\underline{r}^{i}, t^{-i}\right) \mid \underline{r}^{i}, t^{-i}\right)-v^{i}\left(f\left(r^{i}, t^{-i}\right) \mid \underline{r}^{i}, t^{-i}\right)\right] .
\end{aligned}
$$

Notice that the first and the last term on the left-hand side of the inequality cancel. Hence

$$
\begin{array}{ll} 
& E_{-i}\left[v^{i}\left(f\left(\underline{r}^{i}, t^{-i}\right) \mid \underline{r}^{i}, t^{-i}\right)-v^{i}\left(f\left(\bar{r}^{i}, t^{-i}\right) \mid \underline{r}^{i}, t^{-i}\right)\right] \\
\geq & E_{-i}\left[v^{i}\left(f\left(\underline{r}^{i}, t^{-i}\right) \mid \underline{r}^{i}, t^{-i}\right)-v^{i}\left(f\left(r^{i}, t^{-i}\right) \mid \underline{r}^{i}, t^{-i}\right)\right] \\
& +E_{-i}\left[v^{i}\left(f\left(r^{i}, t^{-i}\right) \mid r^{i}, t^{-i}\right)-v^{i}\left(f\left(\bar{r}^{i}, t^{-i}\right) \mid r^{i}, t^{-i}\right)\right] .
\end{array}
$$

Using (2.8) this can be written as

$$
l^{i}\left(\underline{r}^{i}, \bar{r}^{i}\right) \geq l^{i}\left(\underline{r}^{i}, r^{i}\right)+l^{i}\left(r^{i}, \bar{r}^{i}\right)
$$

so the costs of manipulation are decomposition monotone. 


\subsection{Sufficiency of Monotonicity}

As mentioned in Section 2.2, monotonicity is a necessary condition for Bayes-Nash incentive compatibility. In this section we show that this condition is also sufficient if decomposition monotonicity (see Definition 2.4) is satisfied. As demonstrated earlier, monotonicity corresponds to the absence of negative length 2-cycles in $T_{f}^{i}, \forall i \in N$ (see Section 2.3). In order to establish sufficiency, we show that this implies that there do not exist any cycles with negative length. Bayes-Nash incentive compatibility then follows from Theorem 2.1.

Theorem 2.2 Suppose that the allocation rule $f$ and agents'valuation functions are such that the costs of manipulation satisfy decomposition monotonicity. Then, $f$ is Bayes-Nash incentive compatible if and only if $f$ satisfies monotonicity.

\section{Proof}

The necessity of monotonicity follows trivially (see Section 2.2). For the other direction suppose that decomposition monotonicity is satisfied and $f$ satisfies monotonicity.

Take some agent $i$ and let $C=\left(r_{1}^{i}, \ldots, r_{m}^{i}, r_{m+1}^{i}=r_{1}^{i}\right)$ denote a cycle in $T_{f}^{i}$. Whenever an edge of $C$ connects two non-neighboring nodes, we substitute this edge with a path connecting the same two nodes via edges that have the same direction and only connect neighboring nodes. By doing this we generate a new cycle $\tilde{C}$ that has the same nodes as $C$ but consists only of edges between neighboring nodes. An example of this procedure is given in Figure 2.3.

Decomposition monotonicity implies that the length of $C$ is larger than or equal to the length of $\tilde{C}$. Since $\tilde{C}$ is a cycle, we know that at each node the number of edges entering equals the number of edges leaving. This implies that the length of $\tilde{C}$ can be written as the sum of 2 -cycle lengths. Since $f$ satisfies monotonicity there are no 2-cycles with negative length. It follows that $C$ has non-negative length as well.

Finally, absence of cycles with negative length in $T_{f}^{i}, \forall i \in N$ implies that $f$ is Bayes-Nash incentive compatible (see Theorem 2.1).

If agents' valuation functions satisfy non-decreasing expected differences we can state directly the following:

Theorem 2.3 Suppose that agents'valuation functions satisfy non-decreasing expected differences. Then, the allocation rule $f$ is Bayes-Nash incentive compatible if and only if $f$ satisfies monotonicity. 

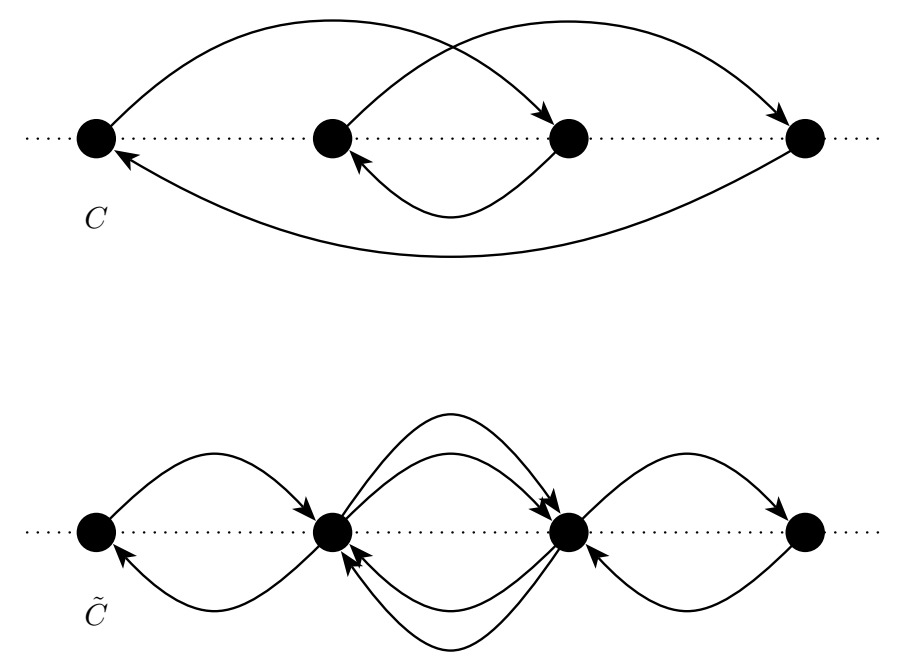

Figure 2.3: The original cycle $C$ and the newly generated cycle $\tilde{C}$.

\section{Proof}

Again, the necessity of monotonicity follows trivially (see Section 2.2). In order to establish sufficiency, note that monotonicity together with non-decreasing expected differences implies that the costs of manipulation are decomposition monotone, see Lemma 2.1. Finally, we apply Theorem 2.2.

Note that monotonicity of the allocation rule $f$ is not a sufficient condition for Bayes-Nash incentive compatibility if the cost of manipulation are not decomposition monotone. This is illustrated in the following example where we present a very simple setting and two allocation rules. In both cases decomposition monotonicity is violated and monotonicity is satisfied. However, one allocation rule is Bayes-Nash incentive compatible while the other one is not.

Example 2.1 For simplicity we assume that there exists only a single agent. His type space $T=$ $\{x, y, z\}$ consists of three types $x, y, z \in \mathbb{R}$, for which we assume $x<y<z$. There are three possible outcomes, specifically $\Gamma=\{a, b, c\}$. The agent's valuations for the different outcomes, depending on his type, are stated in Table 2.1.

Let us first consider the allocation rule $f$ which is defined as follows: $f(x)=b, f(y)=c, f(z)=a$. So, if the agent reports truthfully his type, the allocation rule assigns to him his most preferred outcome. The corresponding network $T_{f}$ is depicted in Figure 2.4 (for the definition of the edge 


\begin{tabular}{|c||c|c|c|}
\hline \multicolumn{1}{|c||}{ Outcome } & \multicolumn{3}{c|}{ Agent's type } \\
\cline { 2 - 4 }$\gamma$ & $x$ & $y$ & $z$ \\
\hline \hline \multirow{2}{*}{$a$} & 2 & 0 & 3 \\
\cline { 2 - 4 }$b$ & 3 & 2 & 0 \\
\cline { 2 - 4 }$c$ & 0 & 3 & 2 \\
\hline
\end{tabular}

Table 2.1: The agent's valuations in Example 2.1.

length see (2.8)). Obviously decomposition monotonicity is not satisfied as $l(x, z)<l(x, y)+l(y, z)$. As easily can be checked, all 2-cycles have length 4, so monotonicity is satisfied. Actually, all cycles have positive length. Hence, by Theorem 2.1 we have that $f$ is Bayes-Nash incentive compatible.

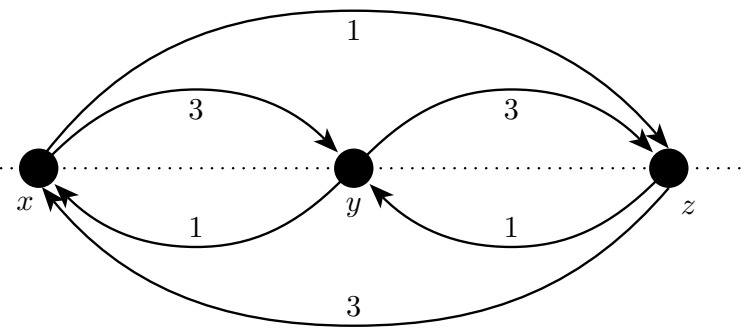

Figure 2.4: The network $T_{f}$ in Example 2.1.

Now let us consider another allocation rule $\hat{f}$ which is defined as follows: $\hat{f}(x)=a, \hat{f}(y)=b$, $\hat{f}(z)=c$. That is, if the agent reports truthfully his type, the allocation rule assigns to him his second most preferred outcome. The corresponding network $T_{\hat{f}}$ is depicted in Figure 2.5. Again decomposition monotonicity is not satisfied as $l(z, x)<l(z, y)+l(y, x)$. As easily can be checked, all 2-cycles have length 1, so monotonicity is satisfied. However, the 3-cycle $C=(x, y, z, x)$ has length $l(x, y)+l(y, z)+l(z, x)=-3$. The existence of such a negative length cycle implies that $f$ is not Bayes-Nash incentive compatible (see Theorem 2.1).

\subsection{Payment Rules}

In the foregoing section we characterized Bayes-Nash incentive compatible allocation rules in terms of a monotonicity condition. In this section we start out by showing how to construct a corresponding payment rule for an incentive compatible allocation rule. Afterwards we discuss the issue of revenue equivalence. 


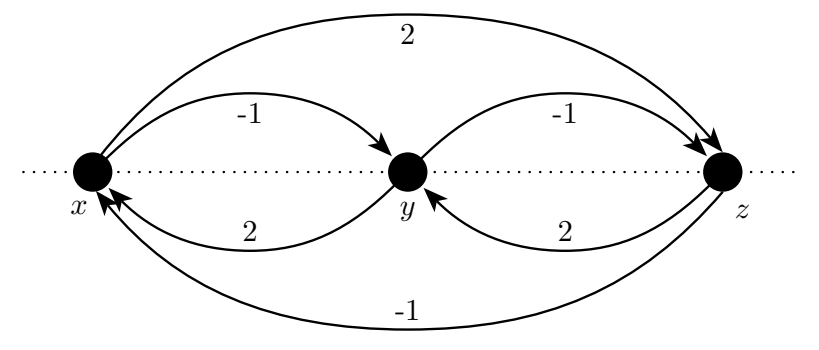

Figure 2.5: The network $T_{\hat{f}}$ in Example 2.1.

According to Definition 2.1 an allocation rule $f$ is Bayes-Nash incentive compatible if there exists a payment rule $P$ such that all the incentive compatibility constraints (2.3) are satisfied. With Theorem 2.1 (see Section 2.3) we establish that the existence of such a payment rule can be guaranteed if for all agents the corresponding networks $T_{f}^{i}$ do not contain any negative length cycles. In the proof of the theorem we construct a payment rule by making use of shortest path lengths. Specifically, for every agent $i$ we pick an arbitrary source node $r_{0}^{i}$ and define for all nodes $r^{i}$

$$
p^{i}\left(r^{i}\right)=\inf \sum_{j=1}^{m} l^{i}\left(r_{j}^{i}, r_{j+1}^{i}\right),
$$

where the infimum is taken over all paths $A=\left(r_{1}^{i}=r^{i}, \ldots, r_{m+1}^{i}=r_{0}^{i}\right)$ in $T_{f}^{i}$, that is, all paths that start at node $r^{i}$ and end at the source node $r_{0}^{i}$. The payment rule $P$ is then constructed by setting

$$
P_{i}\left(r^{i}, t^{-i}\right)=p^{i}\left(r^{i}\right) \quad \forall t^{-i} \in T^{-i}
$$

Thus, the payment agent $i$ has to make for reporting $r^{i}$ corresponds to the length of the shortest path in $T_{f}^{i}$ from the node $r^{i}$ to the source node.

As noted at the end of the proof for Theorem 2.1, it is actually sufficient to choose a payment rule such that

$$
E_{-i}\left[P_{i}\left(r^{i}, t^{-i}\right)\right]=p^{i}\left(r^{i}\right)+c^{i}
$$

That is, the expected payment agent $i$ has to make for reporting $r^{i}$ corresponds to the length of the shortest path from the node $r^{i}$ to the source node plus possibly some constant. Thus, we can observe that there is room for a variety of payment rules that make the allocation rule $f$ Bayes-Nash incentive 
compatible. First of all, even for a fixed source node there can exist several payment rules yielding the same expected payments up to an additive constant. Secondly, changing the source node might lead to payment rules yielding different expected payments. Thirdly, there might exist payment rules that do not evolve from shortest path lengths. The setting described in Section 2.5.2 serves as an illustrative example for the later two sources of variety.

In the remainder of this section we establish conditions under which we can guarantee that actually all payment rules that make an allocation rule $f$ Bayes-Nash incentive compatible yield the same expected payments for the agents up to an additive constant. Such an allocation rule is said to be satisfying revenue equivalence. Krishna and Maenner (2001) show that if agents' type spaces are convex and their valuation functions are convex in their own types then every BayesNash incentive compatible allocation rule satisfies revenue equivalence. Heydenreich, Müller, Uetz and Vohra (2009) are concerned with dominant strategy incentive compatible allocation rules and the characterization of revenue equivalence based on a graph theoretic argument. In an application section they establish that their approach has a natural extension to other notions of incentive compatibility as well. Specifically, they show that a Bayes-Nash incentive compatible allocation rule satisfies revenue equivalence if and only if for every agent $i$ and all types $s^{i}, t^{i} \in T^{i}$ the length of the shortest path from $s^{i}$ to $t^{i}$ and the length of the shortest path from $t^{i}$ to $s^{i}$ add up to zero. Furthermore, Heydenreich, Müller, Uetz and Vohra (2009) establish that the above mentioned result of Krishna and Maenner (2001) follows as an immediate consequence of their results when the outcome space is countable.

In the following we show under the same conditions as Krishna and Maenner (2001) that all Bayes-Nash incentive compatible allocation rules satisfy revenue equivalence. Although we do not extend their result, we give an alternate proof using our network based approach. We also present illustrative example settings in which we relax these conditions. In these settings there exist several payment rules which make $f$ Bayes-Nash incentive compatible, yet do not yield the same expected payments up to an additive constant.

We begin with the definition of revenue equivalence:

Definition 2.5 (Revenue Equivalence) Suppose the allocation rule $f$ is Bayes-Nash incentive compatible. We say that $f$ satisfies revenue equivalence if for any two payment rules $P, \tilde{P}$ such that all the incentive compatibility constraints (2.3) are satisfied there exists a constant $c^{i}$ such that we have

$$
E_{-i}\left[P_{i}\left(t^{i}, t^{-i}\right)\right]=E_{-i}\left[\tilde{P}_{i}\left(t^{i}, t^{-i}\right)\right]+c^{i} \quad \forall t^{i} \in T^{i}, \forall i \in N
$$


So, an allocation rule $f$ satisfies revenue equivalence if all payment rules that make $f$ Bayes-Nash incentive compatible yield the same expected payments up to an additive constant. Our notion of revenue equivalence is equivalent to what is termed payoff equivalence in Krishna and Maenner (2001).

Suppose that the allocation rule $f$ is Bayes-Nash incentive compatible. Furthermore, suppose that $P$ is a payment rule such that all the incentive constraints in (2.3) are satisfied. Now let us consider agent $i$ and some types $s^{i}, t^{i} \in T^{i}$. Based on (2.3) and (2.8) we have that

$$
E_{-i}\left[P_{i}\left(s^{i}, t^{-i}\right)-P_{i}\left(t^{i}, t^{-i}\right)\right] \leq l^{i}\left(s^{i}, t^{i}\right)
$$

as well as that

$$
E_{-i}\left[P_{i}\left(t^{i}, t^{-i}\right)-P_{i}\left(s^{i}, t^{-i}\right)\right] \leq l^{i}\left(t^{i}, s^{i}\right)
$$

Together the above inequalities yield

$$
-l^{i}\left(t^{i}, s^{i}\right) \leq E_{-i}\left[P_{i}\left(s^{i}, t^{-i}\right)-P_{i}\left(t^{i}, t^{-i}\right)\right] \leq l^{i}\left(s^{i}, t^{i}\right) .
$$

That is, for agent $i$ the difference in expected payments for reporting $s^{i}$ or $t^{i}$ is bounded by the length of the edge from $s^{i}$ to $t^{i}$ and the negative length of the backward edge.

Now consider a sequence of nodes between $s^{i}$ and $t^{i}, A=\left(r_{1}^{i}=s^{i}, \ldots, r_{m}^{i}, r_{m+1}^{i}=t^{i}\right)$. Parallel to the above we have for any $r_{j}^{i}, r_{j+1}^{i}$ in $A$ that

$$
E_{-i}\left[P_{i}\left(r_{j}^{i}, t^{-i}\right)-P_{i}\left(r_{j+1}^{i}, t^{-i}\right)\right] \leq l^{i}\left(r_{j}^{i}, r_{j+1}^{i}\right)
$$

and that

$$
E_{-i}\left[P_{i}\left(r_{j+1}^{i}, t^{-i}\right)-P_{i}\left(r_{j}^{i}, t^{-i}\right)\right] \leq l^{i}\left(r_{j+1}^{i}, r_{j}^{i}\right)
$$

Similar to (2.9) together this yields

$$
-l^{i}\left(r_{j+1}^{i}, r_{j}^{i}\right) \leq E_{-i}\left[P_{i}\left(r_{j}^{i}, t^{-i}\right)-P_{i}\left(r_{j+1}^{i}, t^{-i}\right)\right] \leq l^{i}\left(r_{j}^{i}, r_{j+1}^{i}\right) .
$$

By summing up the inequalities (2.10) for all elements in $A$ we get

$$
\sum_{j=1}^{m}-l^{i}\left(r_{j+1}^{i}, r_{j}^{i}\right) \leq \sum_{j=1}^{m} E_{-i}\left[P_{i}\left(r_{j}^{i}, t^{-i}\right)-P_{i}\left(r_{j+1}^{i}, t^{-i}\right)\right] \leq \sum_{j=1}^{m} l^{i}\left(r_{j}^{i}, r_{j+1}^{i}\right) .
$$


Observe that

$$
\begin{aligned}
\sum_{j=1}^{m} E_{-i}\left[P_{i}\left(r_{j}^{i}, t^{-i}\right)-P_{i}\left(r_{j+1}^{i}, t^{-i}\right)\right] & =E_{-i}\left[P_{i}\left(r_{1}^{i}, t^{-i}\right)-P_{i}\left(r_{m+1}^{i}, t^{-i}\right)\right] \\
& =E_{-i}\left[P_{i}\left(s^{i}, t^{-i}\right)-P_{i}\left(t^{i}, t^{-i}\right)\right]
\end{aligned}
$$

since all the terms of the summation cancel except for the first one and the last one and since we have that $r_{1}^{i}=s^{i}$ and $r_{m+1}^{i}=t^{i}$. Now, using (2.12) we can write (2.11) as

$$
\sum_{j=1}^{m}-l^{i}\left(r_{j+1}^{i}, r_{j}^{i}\right) \leq E_{-i}\left[P_{i}\left(s^{i}, t^{-i}\right)-P_{i}\left(t^{i}, t^{-i}\right)\right] \leq \sum_{j=1}^{m} l^{i}\left(r_{j}^{i}, r_{j+1}^{i}\right)
$$

So for any sequence of nodes between $s^{i}$ and $t^{i}$, the difference in agent $i$ 's expected payments for reporting $s^{i}$ or $t^{i}$ is bounded by the length of the path from $s^{i}$ to $t^{i}$ along these nodes and the negative length of the reverse path along the same nodes. Under certain conditions, stated in Theorem 2.4, we can guarantee that there exists a sequence of nodes such that the left-hand side and the right-hand side of (2.13) are equal and hence, the allocation rule $f$ satisfies revenue equivalence.

Before we state the main result of this section, let us introduce the following helpful result. Suppose that agent $i$ 's expected valuation functions $E_{-i}\left[v^{i}\left(f\left(r^{i}, t^{-i}\right) \mid ., t^{-i}\right)\right]$ are convex in his true type. Let $\partial E_{-i}\left[v^{i}\left(f\left(r^{i}, t^{-i}\right) \mid t^{i}, t^{-i}\right)\right]$ denote the set of all subgradients of $E_{-i}\left[v^{i}\left(f\left(r^{i}, t^{-i}\right) \mid ., t^{-i}\right)\right]$ at $t^{i}$. Note that $\partial E_{-i}\left[v^{i}\left(f\left(r^{i}, t^{-i}\right) \mid t^{i}, t^{-i}\right)\right]$ is a non-empty set for all $t^{i} \in T^{i}$ due to the convexity of $E_{-i}\left[v^{i}\left(f\left(r^{i}, t^{-i}\right) \mid ., t^{-i}\right)\right]$, see for example Rockafellar (1970), p.218. For every $r^{i} \in T^{i}$ we pick an element of $\partial E_{-i}\left[v^{i}\left(f\left(r^{i}, t^{-i}\right) \mid r^{i}, t^{-i}\right)\right]$ which we denote by $x^{i}\left(r^{i}\right)$. That is,

$$
x^{i}\left(r^{i}\right) \in \partial E_{-i}\left[v^{i}\left(f\left(r^{i}, t^{-i}\right) \mid r^{i}, t^{-i}\right)\right] \quad \forall r^{i} \in T^{i} .
$$

Lemma 2.2 Suppose that agent $i$ 's expected valuation functions $E_{-i}\left[v^{i}\left(f\left(r^{i}, t^{-i}\right) \mid ., t^{-i}\right)\right]$ are convex in his true type. If the allocation rule $f$ satisfies monotonicity then $x^{i}($.$) is monotonically in-$ creasing.

\section{Proof}

Let us assume that agent $i$ has expected valuation functions which are convex in his true type. Furthermore, let us assume that the allocation rule $f$ is monotone. 
Consider any two types $s^{i}, t^{i} \in T^{i}$. Since the allocation rule $f$ is monotone we have that

$$
l^{i}\left(s^{i}, t^{i}\right)+l^{i}\left(t^{i}, s^{i}\right) \geq 0
$$

Using the edge length definition (2.8) this can be written as

$$
\begin{aligned}
& E_{-i}\left[v^{i}\left(f\left(s^{i}, t^{-i}\right) \mid s^{i}, t^{-i}\right)-v^{i}\left(f\left(s^{i}, t^{-i}\right) \mid t^{i}, t^{-i}\right)\right] \\
& +E_{-i}\left[v^{i}\left(f\left(t^{i}, t^{-i}\right) \mid t^{i}, t^{-i}\right)-v^{i}\left(f\left(t^{i}, t^{-i}\right) \mid s^{i}, t^{-i}\right)\right] \\
\geq & 0 .
\end{aligned}
$$

Note that by definition of the subgradient

$$
E_{-i}\left[v^{i}\left(f\left(s^{i}, t^{-i}\right) \mid t^{i}, t^{-i}\right)-v^{i}\left(f\left(s^{i}, t^{-i}\right) \mid s^{i}, t^{-i}\right)\right] \geq\left(t^{i}-s^{i}\right) x^{i}\left(s^{i}\right)
$$

and

$$
E_{-i}\left[v^{i}\left(f\left(t^{i}, t^{-i}\right) \mid s^{i}, t^{-i}\right)-v^{i}\left(f\left(t^{i}, t^{-i}\right) \mid t^{i}, t^{-i}\right)\right] \geq\left(s^{i}-t^{i}\right) x^{i}\left(t^{i}\right)
$$

Multiplying both sides by -1 yields

$$
E_{-i}\left[v^{i}\left(f\left(s^{i}, t^{-i}\right) \mid s^{i}, t^{-i}\right)-v^{i}\left(f\left(s^{i}, t^{-i}\right) \mid t^{i}, t^{-i}\right)\right] \leq\left(s^{i}-t^{i}\right) x^{i}\left(s^{i}\right)
$$

and

$$
E_{-i}\left[v^{i}\left(f\left(t^{i}, t^{-i}\right) \mid t^{i}, t^{-i}\right)-v^{i}\left(f\left(t^{i}, t^{-i}\right) \mid s^{i}, t^{-i}\right)\right] \leq\left(t^{i}-s^{i}\right) x^{i}\left(t^{i}\right) .
$$

Together with (2.15) the two inequalities above imply that

$$
\left(s^{i}-t^{i}\right)\left(x^{i}\left(s^{i}\right)-x^{i}\left(t^{i}\right)\right) \geq 0 .
$$

Thus, $x^{i}($.$) is monotonically increasing.$

Using Lemma 2.2, we can now state the main result of this section.

Theorem 2.4 Suppose that every agent $i$ has a convex type space $T^{i}$ and expected valuation functions $E_{-i}\left[v^{i}\left(f\left(r^{i}, t^{-i}\right) \mid ., t^{-i}\right)\right]$ which are convex in his true type. If the allocation rule $f$ is Bayes-Nash incentive compatible then it also satisfies revenue equivalence.

\section{Proof}


Let us assume that each agent has a convex type space and expected valuation functions which are convex in his true type. Furthermore, let us assume that the allocation rule $f$ is Bayes-Nash incentive compatible. Let $P$ be a payment rule such that all the incentive compatibility constraints in $(2.3)$ are satisfied.

Take agent $i$ and pick two types $s^{i}, t^{i} \in T^{i}$. Consider a sequence of nodes between $s^{i}$ and $t^{i}$, $A=\left(r_{1}^{i}=s^{i}, \ldots, r_{m}^{i}, r_{m+1}^{i}=t^{i}\right)$. Recall from (2.13) that

$$
\sum_{j=1}^{m}-l^{i}\left(r_{j+1}^{i}, r_{j}^{i}\right) \leq E_{-i}\left[P_{i}\left(s^{i}, t^{-i}\right)-P_{i}\left(t^{i}, t^{-i}\right)\right] \leq \sum_{j=1}^{m} l^{i}\left(r_{j}^{i}, r_{j+1}^{i}\right) .
$$

Note that

$$
\begin{aligned}
\sum_{j=1}^{m}-l^{i}\left(r_{j+1}^{i}, r_{j}^{i}\right)= & \sum_{j=1}^{m} E_{-i}\left[v^{i}\left(f\left(r_{j}^{i}, t^{-i}\right) \mid r_{j+1}^{i}, t^{-i}\right)-v^{i}\left(f\left(r_{j+1}^{i}, t^{-i}\right) \mid r_{j+1}^{i}, t^{-i}\right)\right] \\
= & E_{-i}\left[v^{i}\left(f\left(r_{1}^{i}, t^{-i}\right) \mid r_{2}^{i}, t^{-i}\right)-v^{i}\left(f\left(r_{m+1}^{i}, t^{-i}\right) \mid r_{m+1}^{i}, t^{-i}\right)\right] \\
& +\sum_{j=2}^{m} E_{-i}\left[v^{i}\left(f\left(r_{j}^{i}, t^{-i}\right) \mid r_{j+1}^{i}, t^{-i}\right)-v^{i}\left(f\left(r_{j}^{i}, t^{-i}\right) \mid r_{j}^{i}, t^{-i}\right)\right] \\
= & E_{-i}\left[v^{i}\left(f\left(r_{1}^{i}, t^{-i}\right) \mid r_{1}^{i}, t^{-i}\right)-v^{i}\left(f\left(r_{m+1}^{i}, t^{-i}\right) \mid r_{m+1}^{i}, t^{-i}\right)\right] \\
& +\sum_{j=1}^{m} E_{-i}\left[v^{i}\left(f\left(r_{j}^{i}, t^{-i}\right) \mid r_{j+1}^{i}, t^{-i}\right)-v^{i}\left(f\left(r_{j}^{i}, t^{-i}\right) \mid r_{j}^{i}, t^{-i}\right)\right] \\
= & E_{-i}\left[v^{i}\left(f\left(s^{i}, t^{-i}\right) \mid s^{i}, t^{-i}\right)-v^{i}\left(f\left(t^{i}, t^{-i}\right) \mid t^{i}, t^{-i}\right)\right] \\
& +\sum_{j=1}^{m} E_{-i}\left[v^{i}\left(f\left(r_{j}^{i}, t^{-i}\right) \mid r_{j+1}^{i}, t^{-i}\right)-v^{i}\left(f\left(r_{j}^{i}, t^{-i}\right) \mid r_{j}^{i}, t^{-i}\right)\right] .
\end{aligned}
$$

The first equality follows from the definition of the edge length given in (2.8). The second equality stems from rearranging the terms of the summation. The third equality is derived by adding and subtracting $E_{-i}\left[v^{i}\left(f\left(r_{1}^{i}, t^{-i}\right) \mid r_{1}^{i}, t^{-i}\right)\right]$. To establish the last equality we use that $r_{1}^{i}=s^{i}$ and $r_{m+1}^{i}=t^{i}$. 
In a similarly fashion we get that

$$
\begin{aligned}
\sum_{j=1}^{m} l^{i}\left(r_{j}^{i}, r_{j+1}^{i}\right)= & \sum_{j=1}^{m} E_{-i}\left[v^{i}\left(f\left(r_{j}^{i}, t^{-i}\right) \mid r_{j}^{i}, t^{-i}\right)-v^{i}\left(f\left(r_{j+1}^{i}, t^{-i}\right) \mid r_{j}^{i}, t^{-i}\right)\right] \\
= & E_{-i}\left[v^{i}\left(f\left(r_{1}^{i}, t^{-i}\right) \mid r_{1}^{i}, t^{-i}\right)-v^{i}\left(f\left(r_{m+1}^{i}, t^{-i}\right) \mid r_{m}^{i}, t^{-i}\right)\right] \\
& +\sum_{j=1}^{m-1} E_{-i}\left[v^{i}\left(f\left(r_{j+1}^{i}, t^{-i}\right) \mid r_{j+1}^{i}, t^{-i}\right)-v^{i}\left(f\left(r_{j+1}^{i}, t^{-i}\right) \mid r_{j}^{i}, t^{-i}\right)\right] \\
= & E_{-i}\left[v^{i}\left(f\left(r_{1}^{i}, t^{-i}\right) \mid r_{1}^{i}, t^{-i}\right)-v^{i}\left(f\left(r_{m+1}^{i}, t^{-i}\right) \mid r_{m+1}^{i}, t^{-i}\right)\right] \\
& +\sum_{j=1}^{m} E_{-i}\left[v^{i}\left(f\left(r_{j+1}^{i}, t^{-i}\right) \mid r_{j+1}^{i}, t^{-i}\right)-v^{i}\left(f\left(r_{j+1}^{i}, t^{-i}\right) \mid r_{j}^{i}, t^{-i}\right)\right] \\
= & E_{-i}\left[v^{i}\left(f\left(s^{i}, t^{-i}\right) \mid s^{i}, t^{-i}\right)-v^{i}\left(f\left(t^{i}, t^{-i}\right) \mid t^{i}, t^{-i}\right)\right] \\
& +\sum_{j=1}^{m} E_{-i}\left[v^{i}\left(f\left(r_{j+1}^{i}, t^{-i}\right) \mid r_{j+1}^{i}, t^{-i}\right)-v^{i}\left(f\left(r_{j+1}^{i}, t^{-i}\right) \mid r_{j}^{i}, t^{-i}\right)\right]
\end{aligned}
$$

Again, the first equality follows from (2.8). The second equality stems from rearranging the terms of the summation. The third equality is derived by adding and subtracting $E_{-i}\left[v^{i}\left(f\left(r_{m+1}^{i}, t^{-i}\right) \mid r_{m+1}^{i}, t^{-i}\right)\right]$. In order to establish the last equality we again use that $r_{1}^{i}=s^{i}$ and $r_{m+1}^{i}=t^{i}$. Using (2.16) and (2.17) we can now write (2.13) as follows

$$
\begin{aligned}
& E_{-i}\left[v^{i}\left(f\left(s^{i}, t^{-i}\right) \mid s^{i}, t^{-i}\right)-v^{i}\left(f\left(t^{i}, t^{-i}\right) \mid t^{i}, t^{-i}\right)\right] \\
& +\sum_{j=1}^{m} E_{-i}\left[v^{i}\left(f\left(r_{j}^{i}, t^{-i}\right) \mid r_{j+1}^{i}, t^{-i}\right)-v^{i}\left(f\left(r_{j}^{i}, t^{-i}\right) \mid r_{j}^{i}, t^{-i}\right)\right] \\
\leq & E_{-i}\left[P_{i}\left(s^{i}, t^{-i}\right)-P_{i}\left(t^{i}, t^{-i}\right)\right] \\
\leq & E_{-i}\left[v^{i}\left(f\left(s^{i}, t^{-i}\right) \mid s^{i}, t^{-i}\right)-v^{i}\left(f\left(t^{i}, t^{-i}\right) \mid t^{i}, t^{-i}\right)\right] \\
& +\sum_{j=1}^{m} E_{-i}\left[v^{i}\left(f\left(r_{j+1}^{i}, t^{-i}\right) \mid r_{j+1}^{i}, t^{-i}\right)-v^{i}\left(f\left(r_{j+1}^{i}, t^{-i}\right) \mid r_{j}^{i}, t^{-i}\right)\right] .
\end{aligned}
$$

By definition of the subgradient we have for any $r_{j}^{i}, r_{j+1}^{i}$ in $A$ that

$$
E_{-i}\left[v^{i}\left(f\left(r_{j}^{i}, t^{-i}\right) \mid r_{j+1}^{i}, t^{-i}\right)-v^{i}\left(f\left(r_{j}^{i}, t^{-i}\right) \mid r_{j}^{i}, t^{-i}\right)\right] \geq\left(r_{j+1}^{i}-r_{j}^{i}\right) x^{i}\left(r_{j}^{i}\right)
$$

(for definition of $x^{i}($.$) see (2.14)). This implies that$

$$
\sum_{j=1}^{m} E_{-i}\left[v^{i}\left(f\left(r_{j}^{i}, t^{-i}\right) \mid r_{j+1}^{i}, t^{-i}\right)-v^{i}\left(f\left(r_{j}^{i}, t^{-i}\right) \mid r_{j}^{i}, t^{-i}\right)\right] \geq \sum_{j=1}^{m}\left(r_{j+1}^{i}-r_{j}^{i}\right) x^{i}\left(r_{j}^{i}\right)
$$


Similarly we have that

$$
E_{-i}\left[v^{i}\left(f\left(r_{j+1}^{i}, t^{-i}\right) \mid r_{j}^{i}, t^{-i}\right)-v^{i}\left(f\left(r_{j+1}^{i}, t^{-i}\right) \mid r_{j+1}^{i}, t^{-i}\right)\right] \geq\left(r_{j}^{i}-r_{j+1}^{i}\right) x^{i}\left(r_{j+1}^{i}\right) .
$$

Multiplying both sides by -1 yields

$$
E_{-i}\left[v^{i}\left(f\left(r_{j+1}^{i}, t^{-i}\right) \mid r_{j+1}^{i}, t^{-i}\right)-v^{i}\left(f\left(r_{j+1}^{i}, t^{-i}\right) \mid r_{j}^{i}, t^{-i}\right)\right] \leq\left(r_{j+1}^{i}-r_{j}^{i}\right) x^{i}\left(r_{j+1}^{i}\right)
$$

which implies that

$$
\sum_{j=1}^{m} E_{-i}\left[v^{i}\left(f\left(r_{j+1}^{i}, t^{-i}\right) \mid r_{j+1}^{i}, t^{-i}\right)-v^{i}\left(f\left(r_{j+1}^{i}, t^{-i}\right) \mid r_{j}^{i}, t^{-i}\right)\right] \leq \sum_{j=1}^{m}\left(r_{j+1}^{i}-r_{j}^{i}\right) x^{i}\left(r_{j+1}^{i}\right)
$$

Applying (2.19) and (2.20) to (2.18) yields

$$
\begin{aligned}
& E_{-i}\left[v^{i}\left(f\left(s^{i}, t^{-i}\right) \mid s^{i}, t^{-i}\right)-v^{i}\left(f\left(t^{i}, t^{-i}\right) \mid t^{i}, t^{-i}\right)\right]+\sum_{j=1}^{m}\left(r_{j+1}^{i}-r_{j}^{i}\right) x^{i}\left(r_{j}^{i}\right) \\
\leq & E_{-i}\left[P_{i}\left(s^{i}, t^{-i}\right)-P_{i}\left(t^{i}, t^{-i}\right)\right] \\
\leq & E_{-i}\left[v^{i}\left(f\left(s^{i}, t^{-i}\right) \mid s^{i}, t^{-i}\right)-v^{i}\left(f\left(t^{i}, t^{-i}\right) \mid t^{i}, t^{-i}\right)\right]+\sum_{j=1}^{m}\left(r_{j+1}^{i}-r_{j}^{i}\right) x^{i}\left(r_{j+1}^{i}\right) .
\end{aligned}
$$

By Lemma $2.2 x^{i}($.$) is monotonically increasing. Hence, x^{i}($.$) is Riemann integrable, see for$ example Khuri (2003), Theorem 6.3.2. From this it follows that

$$
\begin{aligned}
& E_{-i}\left[v^{i}\left(f\left(s^{i}, t^{-i}\right) \mid s^{i}, t^{-i}\right)-v^{i}\left(f\left(t^{i}, t^{-i}\right) \mid t^{i}, t^{-i}\right)\right]+\int_{s^{i}}^{t^{i}} x^{i}\left(r^{i}\right) d r^{i} \\
\leq & E_{-i}\left[P_{i}\left(s^{i}, t^{-i}\right)-P_{i}\left(t^{i}, t^{-i}\right)\right] \\
\leq & E_{-i}\left[v^{i}\left(f\left(s^{i}, t^{-i}\right) \mid s^{i}, t^{-i}\right)-v^{i}\left(f\left(t^{i}, t^{-i}\right) \mid t^{i}, t^{-i}\right)\right]+\int_{s^{i}}^{t^{i}} x^{i}\left(r^{i}\right) d r^{i} .
\end{aligned}
$$

So, we get that for all $s^{i}, t^{i} \in T^{i}$

$$
\begin{aligned}
& E_{-i}\left[P_{i}\left(s^{i}, t^{-i}\right)-P_{i}\left(t^{i}, t^{-i}\right)\right] \\
= & E_{-i}\left[v^{i}\left(f\left(s^{i}, t^{-i}\right) \mid s^{i}, t^{-i}\right)-v^{i}\left(f\left(t^{i}, t^{-i}\right) \mid t^{i}, t^{-i}\right)\right]+\int_{s^{i}}^{t^{i}} x^{i}\left(r^{i}\right) d r^{i} .
\end{aligned}
$$

That is, up to an additive constant, the expected payments of an agent are completely defined by 
his valuation function and the allocation rule $f$. Thus, $f$ satisfies revenue equivalence.

Two conditions needed for Theorem 2.4 are the convexity of agents' type spaces and that agents' expected valuation functions are convex in their types. These conditions cannot easily be relaxed as we demonstrate with two examples. In each setting we relax one of the conditions and show that there exist several payment rules making the allocation rule Bayes-Nash incentive compatible which do not yield the same expected payments up to an additive constant. Hence, the allocation rule does not satisfy revenue equivalence.

\subsubsection{Example Setting: Concave Valuation Functions}

This example is based on Holmström (1979), Appendix B. Heydenreich, Müller, Uetz and Vohra (2009) also give an interpretation by embedding it into a framework of demand rationing problems as discussed in Cachon and Lariviere (1999).

Let us consider a setting in which a perfectly divisible item is distributed. For simplicity we assume that there exists only a single agent with type space $T=[0,1]$. His type reflects his demand for a certain fraction of the item. The allocation rule $f:[0,1] \mapsto[0,1]$ determines what fraction of the item is allocated to the agent. Specifically, we have $f(r)=r$. That is, the allocation rule meets whatever demand is reported by the agent. The agent's valuation for reporting $r$ while having true demand $t$ is as follows (for illustration see Figure 2.6):

$$
v(f(r) \mid t)=\left\{\begin{array}{ll}
0 & \text { if } f(r) \leq t \\
t-f(r) & \text { if } f(r)>t
\end{array} .\right.
$$

Take any $s<t$ in $T$. The corresponding edge lengths in the network $T_{f}$ are

$$
l(s, t)=t-s>0
$$

and

$$
l(t, s)=0
$$

Since all edges in $T_{f}$ have non-negative lengths there do not exist any negative length cycles. Thus, by Theorem 2.1 we have that $f$ is Bayes-Nash incentive compatible. Furthermore, notice that for 


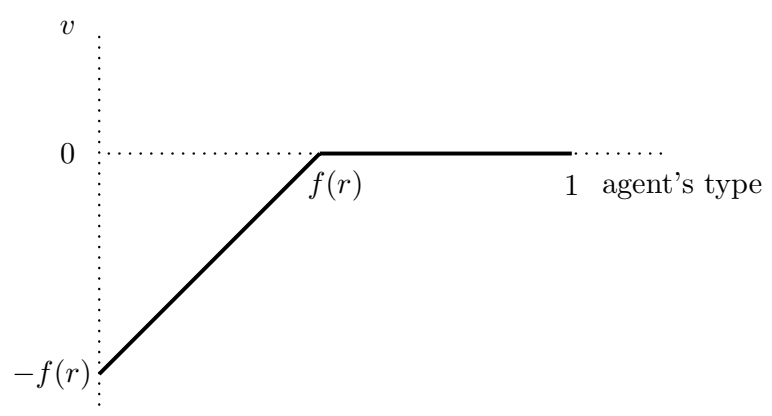

Figure 2.6: The agent's valuations $v(f(r) \mid t)$ for outcome $f(r)$.

any $r$ between $s$ and $t$, that is $s<r<t$, we have

$$
l(s, t)=l(s, r)+l(r, t)=t-s
$$

and

$$
l(t, s)=l(t, r)+l(r, s)=0
$$

This implies that every path from $s$ to $t$ has the same length as the direct edge from $s$ to $t$. Also, every reverse path from $t$ to $s$ has the same length as the direct edge from $t$ to $s$.

Now let us construct a payment rule $P$ that makes the allocation rule $f$ Bayes-Nash incentive compatible. Following the approach based on shortest path lengths as described in the proof of Theorem 2.1 and the beginning of this section, we pick a source node $r_{0}$ in $T_{f}$ and set

$$
P(r)=\inf \sum_{j=1}^{m} l\left(r_{j}, r_{j+1}\right),
$$

where the infimum is taken over all paths $A=\left(r_{1}=r, \ldots, r_{m+1}=r_{0}\right)$ in $T_{f}$, that is, all paths starting at node $r$ and ending at the source node. If we pick $r_{0}=0$ as the source node this yields the payment rule $P(r)=0$. However, if we set $r_{0}=1$ we get a different payment rule, namely $\tilde{P}(r)=1-r$. Clearly, $P$ and $\tilde{P}$ do not yield the same payments up to an additive constant. Hence, the allocation rule $f$ does not satisfy revenue equivalence. 


\subsubsection{Example Setting: Discrete Type Spaces}

Let us consider a setting in which a buyer wants to purchase an item via a procurement auction. There is a set of three potential suppliers for this item, $N=\{1,2,3\}$. Each supplier $i$ has a discrete type space $T^{i}=\{1,2,3\}$. A supplier's type reflects his production cost for the item. Suppliers' types are independently and equally distributed with $\pi^{i}\left(t^{i}\right)=\frac{1}{3}$ for all $t^{i} \in T^{i}$ and $i \in N$.

Based on the suppliers' type reports the winner of the auction is selected as follows: The supplier reporting the lowest type wins. If the lowest type is submitted by several suppliers then the tie is broken by means of an equal chance lottery. In case two suppliers report 3 while the other one reports 1 the low bid is deleted and thus the winner is drawn from the remaining two suppliers. ${ }^{3}$ More formally, given reports from all suppliers, the allocation rule $f: T \mapsto[0,1]^{3}$ assigns to each supplier a probability for winning the contract to deliver the item. So the outcome set $\Gamma$ is the set of all possible winning probability profiles. Supplier $i$ 's probability to win, given a report profile $t \in T$, is denoted $f^{i}(t)$. His winning probabilities for all possible report profiles are stated in Table 2.2.

\begin{tabular}{|c||c|c|c|c|c|c|c|c|c|}
\hline \multicolumn{1}{|c||}{$\begin{array}{c}\text { Report } t^{i} \\
\text { of supplier } i\end{array}$} & \multicolumn{10}{|c|}{ Report profile $t^{-i}$ of the other suppliers } \\
\cline { 2 - 11 } & $\{1,1\}$ & $\{1,2\}$ & $\{1,3\}$ & $\{2,1\}$ & $\{2,2\}$ & $\{2,3\}$ & $\{3,1\}$ & $\{3,2\}$ & $\{3,3\}$ \\
\hline \hline \multirow{2}{*}{1} & $\frac{1}{3}$ & $\frac{1}{2}$ & $\frac{1}{2}$ & $\frac{1}{2}$ & 1 & 1 & $\frac{1}{2}$ & 1 & 0 \\
\cline { 2 - 11 } & 0 & 0 & 0 & 0 & $\frac{1}{3}$ & $\frac{1}{2}$ & 0 & $\frac{1}{2}$ & 1 \\
\cline { 2 - 10 } & 0 & 0 & $\frac{1}{2}$ & 0 & 0 & 0 & $\frac{1}{2}$ & 0 & $\frac{1}{3}$ \\
\hline
\end{tabular}

Table 2.2: Supplier $i$ 's winning probabilities $f^{i}($.$) for the different possible report profiles.$

Suppliers' valuation functions are linear in their own types. Take supplier $i$ having true type $t^{i}$ and reporting $r^{i}$ while the other suppliers have true types $t^{-i}$ and make reports $r^{-i}$. Supplier $i$ 's valuation for the resulting outcome is

$$
v^{i}\left(f\left(r^{i}, r^{-i}\right) \mid t^{i}, t^{-i}\right)=-f^{i}\left(r^{i}, r^{-i}\right) t^{i}
$$

Suppose supplier $i$ reports $r^{i}$ and all other suppliers report their types truthfully. Supplier $i$ 's expected conditional probability for winning the auction is

$$
q^{i}\left(r^{i}\right)=\sum_{t^{-i} \in T^{-i}} f^{i}\left(r^{i}, t^{-i}\right) \pi^{-i}\left(t^{-i}\right)
$$

\footnotetext{
${ }^{3}$ Why having the allocation rule delete the low bid in this case? In this bid constellation the buyer might interpret the low bid as an indication of inferior product quality which would affect him adversely. Or he could see it as an indication of price dumping behavior which he does not want to tolerate, for example because the buyer is a public institution.
} 
This yields $q^{i}(1)=\frac{16}{27}, q^{i}(2)=\frac{7}{27}$ and $q^{i}(3)=\frac{4}{27}$. The supplier's expected valuation is

$$
E_{-i}\left[v^{i}\left(f\left(r^{i}, t^{-i}\right) \mid t^{i}, t^{-i}\right)\right]=-q^{i}\left(r^{i}\right) t^{i}
$$

Take two types $r^{i}, \tilde{r}^{i} \in T^{i}$. Based on (2.8) and (2.22), the length of the edge from $r^{i}$ to $\tilde{r}^{i}$ in the corresponding network $T_{f}^{i}$ is

$$
l^{i}\left(r^{i}, \tilde{r}^{i}\right)=\left(-q^{i}\left(r^{i}\right)+q^{i}\left(\tilde{r}^{i}\right)\right) r^{i}
$$

The complete network is depicted in Figure 2.7. Clearly, there do not exist any negative length cycles

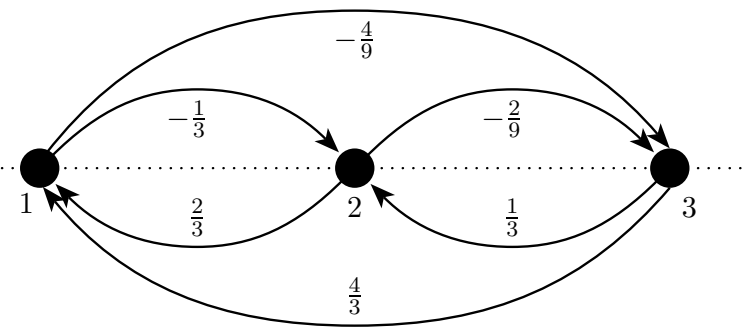

Figure 2.7: The network $T_{f}^{i}$.

in $T_{f}^{i}$. Thus, by Theorem 2.1 we have that $f$ is Bayes-Nash incentive compatible. As a side note we observe that $f$ is not dominant strategy incentive compatible. This would require $\forall s^{i}, t^{i} \in T^{i}$ and $\forall t^{-i} \in T^{-i}$ that

$$
-f^{i}\left(s^{i}, t^{-i}\right) s^{i}-P_{i}\left(s^{i}, t^{-i}\right) \geq-f^{i}\left(t^{i}, t^{-i}\right) s^{i}-P_{i}\left(t^{i}, t^{-i}\right) .
$$

This implies that also

$$
-f^{i}\left(t^{i}, t^{-i}\right) t^{i}-P_{i}\left(t^{i}, t^{-i}\right) \geq-f^{i}\left(s^{i}, t^{-i}\right) t^{i}-P_{i}\left(s^{i}, t^{-i}\right)
$$

Adding the above two inequalities yields

$$
\left(f^{i}\left(t^{i}, t^{-i}\right)-f^{i}\left(s^{i}, t^{-i}\right)\right)\left(t^{i}-s^{i}\right) \leq 0
$$

Thus, it is a necessary condition for dominant strategy incentive compatibility that an agent's winning probability is decreasing in his type for every type profile of the others. Looking at Table 2.2 this condition is obviously violated. 
Next we construct a payment rule $P$ that makes the allocation rule Bayes-Nash incentive compatible. Based on the approach utilizing shortest path lengths which is presented in the proof of Theorem 2.1 and the beginning of this section, we pick a source node $r_{0}^{i}$ in $T_{f}^{i}$ and set

$$
p^{i}\left(r^{i}\right)=\inf \sum_{j=1}^{m} l^{i}\left(r_{j}^{i}, r_{j+1}^{i}\right),
$$

where the infimum is taken over all paths $A=\left(r_{1}^{i}=r^{i}, \ldots, r_{m+1}^{i}=r_{0}^{i}\right)$ in $T_{f}^{i}$ from $r^{i}$ to $r_{0}^{i}$. Any payment rule $P$ such that $E_{-i}\left[P_{i}\left(r^{i}, t^{-i}\right)\right]=p^{i}\left(r^{i}\right)$ makes $f$ Bayes-Nash incentive compatible. This can be achieved for example by setting $P_{i}\left(r^{i}, t^{-i}\right)=p^{i}\left(r^{i}\right), \forall t^{-i} \in T^{-i}$. If we pick $r_{0}^{i}=1$ as the source node this yields

$$
\begin{aligned}
& E_{-i}\left[P_{i}\left(1, t^{-i}\right)\right]=0, \\
& E_{-i}\left[P_{i}\left(2, t^{-i}\right)\right]=\frac{2}{3}, \\
& E_{-i}\left[P_{i}\left(3, t^{-i}\right)\right]=1
\end{aligned}
$$

as expected payments for agent $i$.

Looking at the network $T_{f}^{i}$ (see Figure 2.7) it is obvious that the shortest forward and backward paths between any two nodes do not add up to zero. From Theorem 9 in Heydenreich, Müller, Uetz and Vohra (2009) it follows that the allocation rule $f$ does not satisfy revenue equivalence. That is, there exist payment rules that yield expected payments for the agents which differ not only by an additive constant.

For example, if we set $r_{0}^{i}=3$ we get a different payment rule $\tilde{P}$ with the following expected payments for agent $i$ :

$$
\begin{aligned}
& E_{-i}\left[\tilde{P}_{i}\left(1, t^{-i}\right)\right]=-\frac{5}{9} \\
& E_{-i}\left[\tilde{P}_{i}\left(2, t^{-i}\right)\right]=-\frac{2}{9} \\
& E_{-i}\left[\tilde{P}_{i}\left(3, t^{-i}\right)\right]=0
\end{aligned}
$$

Clearly, $P$ and $\tilde{P}$ do not yield the same expected payments up to an additive constant.

Since agents' type spaces consist of only three types, we can easily observe how much room there actually is for the existence of different payment rules that make the allocation rule Bayes-Nash incentive compatible. The incentive compatibility constraints (2.3) which have to be fulfilled for 
agent $i$ are

$$
\begin{aligned}
-q^{i}(1) 1-E_{-i}\left[P_{i}\left(1, t^{-i}\right)\right] & \geq-q^{i}(2) 1-E_{-i}\left[P_{i}\left(2, t^{-i}\right)\right], \\
-q^{i}(1) 1-E_{-i}\left[P_{i}\left(1, t^{-i}\right)\right] & \geq-q^{i}(3) 1-E_{-i}\left[P_{i}\left(3, t^{-i}\right)\right], \\
-q^{i}(2) 2-E_{-i}\left[P_{i}\left(2, t^{-i}\right)\right] & \geq-q^{i}(1) 2-E_{-i}\left[P_{i}\left(1, t^{-i}\right)\right], \\
-q^{i}(2) 2-E_{-i}\left[P_{i}\left(2, t^{-i}\right)\right] & \geq-q^{i}(3) 2-E_{-i}\left[P_{i}\left(3, t^{-i}\right)\right], \\
-q^{i}(3) 3-E_{-i}\left[P_{i}\left(3, t^{-i}\right)\right] & \geq-q^{i}(1) 3-E_{-i}\left[P_{i}\left(1, t^{-i}\right)\right], \\
-q^{i}(3) 3-E_{-i}\left[P_{i}\left(3, t^{-i}\right)\right] & \geq-q^{i}(2) 3-E_{-i}\left[P_{i}\left(2, t^{-i}\right)\right] .
\end{aligned}
$$

By plugging in the expected conditional winning probabilities that we have computed above and combining (2.23) and (2.25), (2.24) and (2.27) as well as (2.26) and (2.28) we get

$$
\begin{aligned}
-\frac{2}{3} & \leq E_{-i}\left[P_{i}\left(1, t^{-i}\right)-P_{i}\left(2, t^{-i}\right)\right] \leq-\frac{1}{3} \\
-\frac{4}{3} & \leq E_{-i}\left[P_{i}\left(1, t^{-i}\right)-P_{i}\left(3, t^{-i}\right)\right] \leq-\frac{4}{9} \\
-\frac{1}{3} \leq E_{-i}\left[P_{i}\left(2, t^{-i}\right)-P_{i}\left(3, t^{-i}\right)\right] & \leq-\frac{2}{9} .
\end{aligned}
$$

Any payment rule that satisfies the above constraints makes $f$ Bayes-Nash incentive compatible. Of course this leaves also room for payment rules that are not based on shortest paths to some fixed source node. Take for example a payment rule $\hat{P}$ that yields the following expected payments:

$$
\begin{aligned}
& E_{-i}\left[\hat{P}_{i}\left(1, t^{-i}\right)\right]=-\frac{17}{18} \\
& E_{-i}\left[\hat{P}_{i}\left(2, t^{-i}\right)\right]=-\frac{5}{18} \\
& E_{-i}\left[\hat{P}_{i}\left(3, t^{-i}\right)\right]=0
\end{aligned}
$$

\subsection{Applications}

Malakhov and Vohra (2004) consider a multiple item auction setting where agents have finite type spaces and independent valuations. They show that an allocation rule is Bayes-Nash incentive compatible if and only if it satisfies monotonicity.

Myerson (1981) deals with a single item auction setting where agents have convex type spaces and interdependent valuations. Feng (2008) considers a multiple item auction setting where agents have convex type spaces and independent valuations. Both Myerson (1981) and Feng (2008) give a similar 
characterization of Bayes-Nash incentive compatibility in their respective settings. They show that a mechanism $(f, P)$ is Bayes-Nash incentive compatible if and only if monotonicity is satisfied and a condition on agents' utility functions holds. This condition is that for all agents $i$ and all types $s^{i}, t^{i} \in T^{i}$

$$
U^{i}\left(s^{i} \mid s^{i}\right)=U^{i}\left(t^{i} \mid t^{i}\right)+\int_{t^{i}}^{s^{i}} q^{i}(r) d r,
$$

where $U^{i}\left(s^{i} \mid s^{i}\right)$ denotes agent $i$ 's expected utility for truthfully reporting $s^{i}$ (see (2.1) for definition of expected utilities) and $q^{i}(r)$ represents some expected winning probability which depends on the allocation rule. Note that the above can be written as

$$
\begin{aligned}
& E_{-i}\left[P_{i}\left(t^{i}, t^{-i}\right)-P_{i}\left(s^{i}, t^{-i}\right)\right] \\
= & E_{-i}\left[v^{i}\left(f\left(t^{i}, t^{-i}\right) \mid t^{i}, t^{-i}\right)-v^{i}\left(f\left(s^{i}, t^{-i}\right) \mid s^{i}, t^{-i}\right)\right]+\int_{t^{i}}^{s^{i}} q^{i}(r) d r .
\end{aligned}
$$

Thus, the second condition used for characterizing Bayes-Nash incentive compatibility, next to monotonicity, is revenue equivalence.

In the following we show how the aforementioned settings and results can be folded into our framework. Especially, we demonstrate, with regard to Myerson (1981) and Feng (2008), that for the characterization of Bayes-Nash incentive compatible allocation rules one only needs to evoke monotonicity.

\subsubsection{Single, Indivisible Item}

In this application we consider a common single item auctions setting. Specifically, we look at a setting presented by Myerson (1981). In this setting a single, indivisible item is auctioned off to one of several potential buyers. A buyer's type reflects his initial value estimate for the item. Each buyer has a convex type space. Specifically, we assume that $T^{i}=\left[a^{i}, b^{i}\right]$ with $-\infty<a^{i}<b^{i}<\infty$. Given reports from all buyers, the allocation rule $f: T \mapsto[0,1]^{n}$ assigns to each buyer a probability for winning the item. So the outcome set $\Gamma$ is the set of all possible winning probability profiles. Buyer $i$ 's probability to win, given a report profile $t \in T$, is denoted $f^{i}(t)$. The allocation rule must satisfy the probability condition $\sum_{i=1}^{n} f^{i}(t) \leq 1, \forall t \in T$.

Buyers' valuations are interdependent. If a buyer would get to know the value estimate of some other buyer, he would want to revise his own initial value estimate for the item. For instance, suppose the item is a painting and a buyer is uncertain about whether he is dealing with an original or a forgery. Learning that another buyer has a low value estimate, suggesting a tendency towards forgery, 
would incline him to revise his own value estimate downwards. We assume that the buyers make these revisions additively according to $n$ revision effect functions $e^{j}: T^{j} \mapsto \mathbb{R}, j \in N$. If buyer $i$ learns that buyer $j$ has type $t^{j}$, he revises his initial value estimate by adding $e^{j}\left(t^{j}\right)$ to it. Additionally, in order to justify that a buyer's type reflects his initial value estimate for the item, it is assumed that

$$
\int_{T^{j}} e^{j}\left(t^{j}\right) \pi^{j}\left(t^{j}\right) d t^{j}=0
$$

That is, revision effects have an expected value of zero. The stated results do not depend on this assumption. However, without it, the interpretation of buyers' types would change. Also, note that modelling the valuation interdependencies as done above implies that actually all buyers $i \neq j$ revise their initial estimates by the same amount $e^{j}\left(t^{j}\right)$. Without affecting any of the results, one could also allow for buyer specific adjustments by assuming that each buyer $i$ has $n-1$ revision effect functions $e_{i}^{j}: T^{j} \mapsto \mathbb{R}, j \in N, j \neq i$.

Take buyer $i$ having true type $t^{i}$ and reporting $r^{i}$ while the other buyers have true types $t^{-i}$ and make reports $r^{-i}$. The value that the buyer assigns to the resulting allocation is

$$
v^{i}\left(f\left(r^{i}, r^{-i}\right) \mid t^{i}, t^{-i}\right)=f^{i}\left(r^{i}, r^{-i}\right)\left(t^{i}+\sum_{\substack{j \in N \\ j \neq i}} e^{j}\left(t^{j}\right)\right) .
$$

Consider buyer $i$ reporting $r^{i}$ and assume that the other buyers report truthfully. Buyer $i$ 's expected conditional probability for winning the item is

$$
q^{i}\left(r^{i}\right)=\int_{T^{-i}} f^{i}\left(r^{i}, t^{-i}\right) \pi^{-i}\left(t^{-i}\right) d t^{-i}
$$

The buyer's expected valuation is

$$
E_{-i}\left[v^{i}\left(f\left(r^{i}, t^{-i}\right) \mid t^{i}, t^{-i}\right)\right]=q^{i}\left(r^{i}\right) t^{i}+\int_{T^{-i}} f^{i}\left(r^{i}, t^{-i}\right)\left(\sum_{\substack{j \in N \\ j \neq i}} e^{j}\left(t^{j}\right)\right) \pi^{-i}\left(t^{-i}\right) d t^{-i}
$$

Applying (2.29), the monotonicity condition (see Definition 2.2) becomes

$$
\left(q^{i}\left(r^{i}\right)-q^{i}\left(\tilde{r}^{i}\right)\right)\left(r^{i}-\tilde{r}^{i}\right) \geq 0 \quad \forall r^{i}, \tilde{r}^{i} \in T^{i}, \forall i \in N
$$

That is, satisfying monotonicity means that a buyer's expected winning probability is increasing in his type report. 
Myerson (1981) shows that a mechanism $(f, P)$ is Bayes-Nash incentive compatible if and only if (2.30) is satisfied and

$$
U^{i}\left(r^{i} \mid r^{i}\right)=U^{i}\left(a^{i} \mid a^{i}\right)+\int_{a^{i}}^{r^{i}} q^{i}(s) d s \quad \forall r^{i} \in T^{i}, \forall i \in N
$$

where $U^{i}\left(r^{i} \mid r^{i}\right)$ denotes buyer $i$ 's expected utility for truthfully reporting $r^{i}$ (see (2.1) for definition of expected utilities).

As easily can be verified, buyers' valuation functions in this single item auction setting satisfy non-decreasing expected differences (see Definition 2.3). Thus, we can directly apply the results derived earlier in Section 2.4 of this chapter. From Theorem 2.3 it follows that $f$ is Bayes-Nash incentive compatible if and only if the monotonicity condition in (2.30) is satisfied.

Now, suppose that the allocation rule satisfies monotonicity. Let us construct a payment rule that makes $f$ Bayes-Nash incentive compatible. We use the approach utilizing shortest path lengths (see proof of Theorem 2.1 and the beginning of Section 2.5). For each buyer $i$ we pick $a^{i}$, that is his lowest possible type, as the source node in $T_{f}^{i}$. We set

$$
p^{i}\left(r^{i}\right)=\inf \sum_{j=1}^{m} l^{i}\left(r_{j}^{i}, r_{j+1}^{i}\right),
$$

where the infimum is taken over all paths $A=\left(r_{1}^{i}=r^{i}, \ldots, r_{m+1}^{i}=a^{i}\right)$ in $T_{f}^{i}$. Any payment rule $P$ such that $E_{-i}\left[P_{i}\left(r^{i}, t^{-i}\right)\right]=p^{i}\left(r^{i}\right)$ makes $f$ Bayes-Nash incentive compatible. This can be achieved for example by setting $P_{i}\left(r^{i}, t^{-i}\right)=p^{i}\left(r^{i}\right), \forall t^{-i} \in T^{-i}$.

Considering the length of such a path $A$, we find by applying (2.29) to (2.17) that

$$
\begin{aligned}
\sum_{j=1}^{m} l^{i}\left(r_{j}^{i}, r_{j+1}^{i}\right)= & E_{-i}\left[v^{i}\left(f\left(r^{i}, t^{-i}\right) \mid r^{i}, t^{-i}\right)-v^{i}\left(f\left(a^{i}, t^{-i}\right) \mid a^{i}, t^{-i}\right)\right] \\
& +\sum_{j=1}^{m} q^{i}\left(r_{j+1}^{i}\right)\left(r_{j+1}^{i}-r_{j}^{i}\right) .
\end{aligned}
$$

Monotonicity (2.30) implies that $q^{i}$ (.) is Riemann integrable, see for example Khuri (2003), Theorem 6.3.2. Furthermore, decomposition monotonicity is satisfied since buyers' valuation functions satisfy non-decreasing expected differences, see Lemma 2.1. So considering any path $A$ from $r^{i}$ to $a^{i}$, we can construct paths that are shorter (or as long) by letting them visit the same nodes as $A$ and also additional nodes in between (see also example in Figure 2.2). In the limit, as $m \rightarrow \infty$, the distance 
between neighboring nodes goes to zero and

$$
\sum_{j=1}^{m} q^{i}\left(r_{j+1}^{i}\right)\left(r_{j+1}^{i}-r_{j}^{i}\right) \rightarrow \int_{r^{i}}^{a^{i}} q^{i}(s) d s
$$

So, based on the above, we find for our payment rule that

$$
E_{-i}\left[P_{i}\left(r^{i}, t^{-i}\right)\right]=E_{-i}\left[v^{i}\left(f\left(r^{i}, t^{-i}\right) \mid r^{i}, t^{-i}\right)-v^{i}\left(f\left(a^{i}, t^{-i}\right) \mid a^{i}, t^{-i}\right)\right]-\int_{a^{i}}^{r^{i}} q^{i}(s) d s .
$$

Note that by construction $E_{-i}\left[P_{i}\left(a^{i}, t^{-i}\right)\right]=0$. Thus, we can subtract $E_{-i}\left[P_{i}\left(a^{i}, t^{-i}\right)\right]$ on the left-hand side of (2.31), yielding

$$
\begin{aligned}
E_{-i}\left[P_{i}\left(r^{i}, t^{-i}\right)-P_{i}\left(a^{i}, t^{-i}\right)\right]= & E_{-i}\left[v^{i}\left(f\left(r^{i}, t^{-i}\right) \mid r^{i}, t^{-i}\right)-v^{i}\left(f\left(a^{i}, t^{-i}\right) \mid a^{i}, t^{-i}\right)\right] \\
& -\int_{a^{i}}^{r^{i}} q^{i}(s) d s .
\end{aligned}
$$

Since buyers' type spaces are convex and buyers' expected valuation functions are linear in their true types it follows from Theorem 2.4 that the allocation rule satisfies revenue equivalence. That is, all payment rules making $f$ Bayes-Nash incentive compatible yield the same expected payments up to an additive constant. So (2.32) holds for all of them. Thus, by rearranging the terms in (2.32), we get

$$
U^{i}\left(r^{i} \mid r^{i}\right)=U^{i}\left(a^{i} \mid a^{i}\right)+\int_{a^{i}}^{r^{i}} q^{i}(s) d s
$$

as a necessary condition for Bayes-Nash incentive compatibility.

\subsubsection{Multiple Units of a Homogenous Good}

In this application we consider a setting presented by Malakhov and Vohra (2004). In this setting there are $k$ units of an homogenous good being auctioned off to several potential buyers. These buyers exhibit constant marginal valuations for getting additional units of the good. That is, a buyer values the first unit that he gets as much as the second unit, the third unit and so on. This marginal valuation for an additional unit of the good is reflected by a buyer's type. Each buyer has a discrete type space consisting of $h_{i}$ different types. Thus, $T^{i}=\left\{t_{1}^{i}, \ldots, t_{h_{i}}^{i}\right\}$ where without loss of generality $t_{j}^{i}<t_{j+1}^{i}$. Given reports from all buyers, the allocation rule $f: T \mapsto \mathbb{R}_{+}^{n}$ determines how many units of the good each buyer is winning. The quantity allocated to buyer $i$, given a report profile $t \in T$, is denoted $f^{i}(t)$. If $f$ is probabilistic, that is, it maps each reported type profile $t$ into a distribution 
over allocations, then $f^{i}(t)$ denotes the expected quantity buyer $i$ is winning.

Buyers' valuations are independent. Take buyer $i$ having true type $t^{i}$ and reporting $r^{i}$ while the other buyers have true types $t^{-i}$ and make reports $r^{-i}$. The buyer assigns the following value to the resulting allocation

$$
v^{i}\left(f\left(r^{i}, r^{-i}\right) \mid t^{i}, t^{-i}\right)=f^{i}\left(r^{i}, r^{-i}\right) t^{i}
$$

Let us assume that buyer $i$ reports $r^{i}$ and the other buyers report truthfully their types. Then buyer $i$ expects to win

$$
q^{i}\left(r^{i}\right)=\sum_{t^{-i} \in T^{-i}} f^{i}\left(r^{i}, t^{-i}\right) \pi^{-i}\left(t^{-i}\right)
$$

units of the good. Hence, the buyer's expected valuation is

$$
E_{-i}\left[v^{i}\left(f\left(r^{i}, t^{-i}\right) \mid t^{i}, t^{-i}\right)\right]=q^{i}\left(r^{i}\right) t^{i}
$$

Using (2.33), the monotonicity condition (see Definition 2.2) becomes

$$
\left(q^{i}\left(r^{i}\right)-q^{i}\left(\tilde{r}^{i}\right)\right)\left(r^{i}-\tilde{r}^{i}\right) \geq 0 \quad \forall r^{i}, \tilde{r}^{i} \in T^{i}, \forall i \in N
$$

That is, satisfying monotonicity means that a buyer's expected winning quantity is increasing in his type report.

As easily can be verified, buyers' valuation functions in this setting are satisfying non-decreasing expected differences, see Definition 2.3. Thus, we can apply the characterization results derived in Section 2.4. From Theorem 2.3 it follows that the allocation rule $f$ is Bayes-Nash incentive compatible if and only if the monotonicity condition in (2.34) is satisfied. This finding is also established in Malakhov and Vohra (2004).

If the allocation rule satisfies monotonicity we can construct a payment rule that makes $f$ BayesNash incentive compatible by using the shortest path length approach described in the beginning of the foregoing section and in the proof of Theorem 2.1. First, we pick $t_{1}^{i}$ as buyer $i$ 's source node in $T_{f}^{i}$. Next, we set

$$
p^{i}\left(r^{i}\right)=\inf \sum_{j=1}^{m} l^{i}\left(r_{j}^{i}, r_{j+1}^{i}\right),
$$

where the infimum is taken over all paths $A=\left(r_{1}^{i}=r^{i}, \ldots, r_{m+1}^{i}=t_{1}^{i}\right)$ in $T_{f}^{i}$. Any payment rule $P$ such that $E_{-i}\left[P_{i}\left(r^{i}, t^{-i}\right)\right]=p^{i}\left(r^{i}\right)$ makes $f$ Bayes-Nash incentive compatible. One way to achieve this is to set $P_{i}\left(r^{i}, t^{-i}\right)=p^{i}\left(r^{i}\right), \forall t^{-i} \in T^{-i}$. 
Note that in this setting decomposition monotonicity is satisfied since buyers' valuation functions satisfy non-decreasing expected differences, see Lemma 2.1. So if we consider any path $A$ from $r^{i}$ to $t_{1}^{i}$, we can construct paths that are shorter (or as long) by letting them visit the same nodes as $A$ and also additional nodes in between (see also example in Figure 2.2). Thus, the shortest path from $r^{i}$ to $t_{1}^{i}$ is the one going through all the nodes in between, see also Figure 2.8. So, based on this
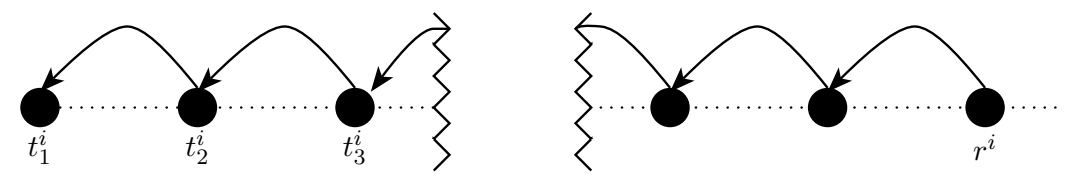

Figure 2.8: Shortest path from $r^{i}$ to the source node $t_{1}^{i}$.

observation, we find that for our payment rule

$$
E_{-i}\left[P_{i}\left(r^{i}, t^{-i}\right)\right]=\sum_{j=1}^{m} l^{i}\left(r_{j}^{i}, r_{j+1}^{i}\right)
$$

where the sum is taken over all edges of the path $\tilde{A}=\left(r_{1}^{i}=r^{i}, \ldots, r_{m}^{i}=t_{2}^{i}, r_{m+1}^{i}=t_{1}^{i}\right)$ visiting every node in between $r^{i}$ and $t_{1}^{i}$. By applying (2.33) to (2.17) the above can be written as

$$
E_{-i}\left[P_{i}\left(r^{i}, t^{-i}\right)\right]=q^{i}\left(r^{i}\right) r^{i}-q^{i}\left(t_{1}^{i}\right) t_{1}^{i}+\sum_{j=1}^{m} q^{i}\left(r_{j+1}^{i}\right)\left(r_{j+1}^{i}-r_{j}^{i}\right)
$$

Finally, we observe that Theorem 2.4 is not applicable in this setting since buyers' type spaces are discrete. So, we cannot generally guarantee that a Bayes-Nash incentive compatible allocation rule also satisfies revenue equivalence. In fact, the discrete type space example in Section 2.5 .2 can be seen as a special case of the setting under concern here. In this example there exist several payment rules that make the allocation rule Bayes-Nash incentive compatible but do not yield the same expected payments up to an additive constant.

\subsubsection{Commonly Ranked Items}

In this section we consider the setting presented by Feng (2008). The analysis of this setting is going to parallel the one of Myerson's (1981) single item setting presented in Section 2.6.1. In Feng's (2008) setting there is a set of items $L=\{1, \ldots, l\}$. These items are auctioned off to $n$ potential buyers. It is assumed that $l \leq n$, so there are no more items then there are buyers. These items are commonly 
ranked by the buyers. Specifically, every buyer values having the first item more than having the second item, which in turn is better than having the third item and so on down to the lth item. As an example for such a scenario one can think of an auction for online advertisement positions on an internet search site like Google or Yahoo. Next to the list of search results the site also presents a list of online ads relating to the search topic. Although different companies might attach different values to the various positions on this list, generally each company values a higher position more than a lower one.

A buyer's type affects his valuation for the different items. Each buyer has a convex type space. Specifically, it is assumed that $T^{i}=\left[\underline{t}^{i}, \bar{t}^{i}\right]$ with $\underline{t}^{i} \geq 0$. Given reports from all buyers, the allocation rule $f: T \mapsto[0,1]^{l n}$ assigns the items to the different buyers. Buyer $i$ 's probability to win item $k$, given a report profile $t \in T$, is denoted $f_{k}^{i}(t)$. The allocation rule must satisfy a couple of probability conditions. First of all, we have $f_{k}^{i}(t) \geq 0, \forall i \in N, \forall k \in L, \forall t \in T$. Furthermore, it is assumed that $\sum_{i=1}^{n} f_{k}^{i}(t) \leq 1, \forall k \in L, \forall t \in T$, so an item is not allocated to more than one buyer. Lastly, we have $\sum_{k=1}^{l} f_{k}^{i}(t) \leq 1, \forall i \in N, \forall t \in T$. That is, a buyer cannot win more than one item.

With regard to buyers' valuation functions, Feng (2008) considers four cases, all of which can be folded into the following. Take buyer $i$ having true type $t^{i}$. The value that $i$ attaches to the $k$ th item is

$$
v^{i}\left(k \mid t^{i}\right)=\alpha(k)+\beta(k) t^{i}
$$

So, buyers' valuations are independent. In addition, a buyer's valuation for a specific item is linear in his type. Note that without affecting any of the results in this section, one could also allow for buyer specific functions $\alpha($.$) and \beta($.$) .$

Concerning the four valuation function cases actually considered by Feng (2008), there is first of all the parallel case in which $\alpha(k)=-a k, a>0$, and $\beta(k)=1$. So, in this case we have

$$
v^{i}\left(k \mid t^{i}\right)=-a k+t^{i}
$$

Thus, if the buyer moves his consideration from a higher ranked item to a lower ranked item, his valuation drops at rate $a$. This drop rate is independent of the buyer's type. Furthermore, Feng (2008) considers three non-parallel cases in which $\alpha(k)=a$ and $\beta(k)=b-k$ with $a+b t^{i}>0$, $\forall t^{i} \in T^{i}$. So, in these cases we have

$$
v^{i}\left(k \mid t^{i}\right)=a+(b-k) t^{i}
$$


In the convergent case we have $b \geq l$, in the divergent case $b \leq 1$ and in the convergent-then-divergent case $1<b<l$. Figure 2.9 illustrates buyer $i$ 's preferences with respect to the ranked items in the four cases described above. Note that (2.35) allows for more general valuation functions than the four kinds analyzed by Feng (2008). Using the framework in (2.35) also helps in showing the links to Myerson's (1981) setting and our results.

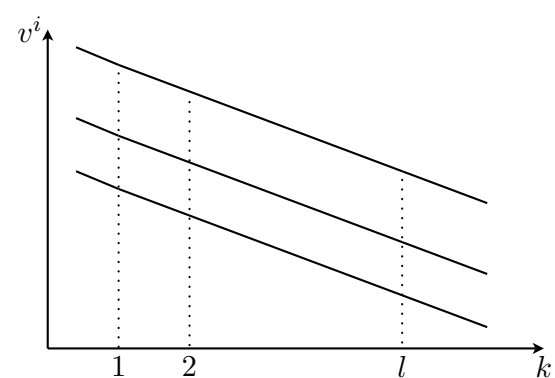

Parallel Case

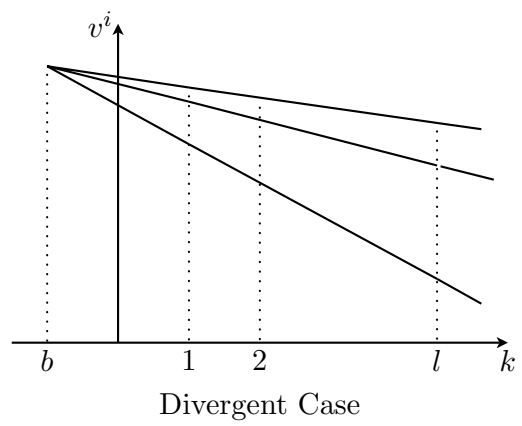

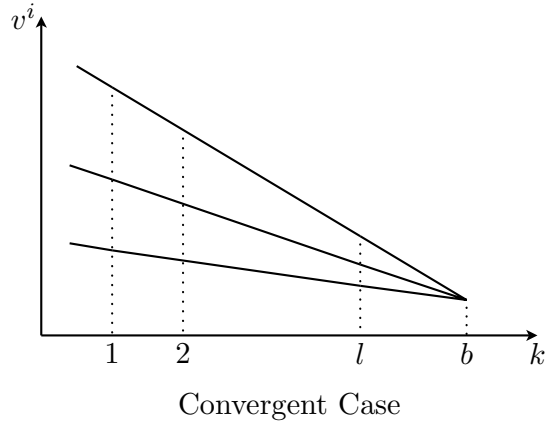

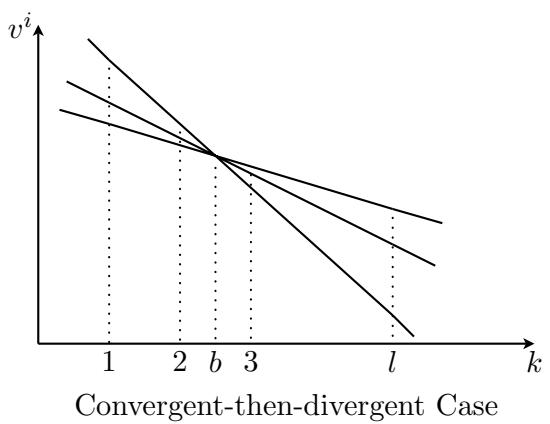

Figure 2.9: Buyer $i$ 's valuation functions $v^{i}\left(. \mid t^{i}\right)$ for different types $t^{i}$ in the four cases described by Feng (2008).

Take buyer $i$ having true type $t^{i}$ and reporting $r^{i}$ while the other buyers have true types $t^{-i}$ and make reports $r^{-i}$. The value that the buyer assigns to the resulting allocation is

$$
v^{i}\left(f\left(r^{i}, r^{-i}\right) \mid t^{i}, t^{-i}\right)=\sum_{k=1}^{l} f_{k}^{i}\left(r^{i}, r^{-i}\right)\left(\alpha(k)+\beta(k) t^{i}\right) .
$$


Suppose buyer $i$ reports $r^{i}$ and the other buyers report truthfully. As done by Feng (2008), we define

$$
q^{i}\left(r^{i}\right)=\int_{T^{-i}}\left(\sum_{k=1}^{l} f_{k}^{i}\left(r^{i}, t^{-i}\right) \beta(k)\right) \pi^{-i}\left(t^{-i}\right) d t^{-i}
$$

as buyer $i$ 's adjusted probability of winning, which is weighted by $\beta(k)$ for different positions $k$. Then, the buyer's expected valuation is

$$
\begin{aligned}
E_{-i}\left[v^{i}\left(f\left(r^{i}, t^{-i}\right) \mid t^{i}, t^{-i}\right)\right] & =\int_{T^{-i}}\left(\sum_{k=1}^{l} f_{k}^{i}\left(r^{i}, t^{-i}\right)\left(\alpha(k)+\beta(k) t^{i}\right)\right) \pi^{-i}\left(t^{-i}\right) d t^{-i} \\
& =q^{i}\left(r^{i}\right) t^{i}+\int_{T^{-i}}\left(\sum_{k=1}^{l} f_{k}^{i}\left(r^{i}, t^{-i}\right) \alpha(k)\right) \pi^{-i}\left(t^{-i}\right) d t^{-i}
\end{aligned}
$$

(2.36) is comparable to the buyer's expected valuation in Myerson's (1981) setting, see (2.29). Both consist of the buyer's true type multiplied by an expected winning probability as well as some additive term that depends on the buyer's report but not on his true type.

As done by Myerson (1981), Feng (2008) shows that a mechanism $(f, P)$ is Bayes-Nash incentive compatible if and only if for every buyer $q^{i}($.$) is increasing in the buyer's type report and$

$$
U^{i}\left(r^{i} \mid r^{i}\right)=U^{i}\left(\underline{t}^{i} \mid \underline{t}^{i}\right)+\int_{\underline{t}^{i}}^{r^{i}} q^{i}(s) d s \quad \forall r^{i} \in T^{i}
$$

where $U^{i}\left(r^{i} \mid r^{i}\right)$ denotes the buyer's expected utility for truthfully reporting $r^{i}$.

As the analysis of this setting parallels the one of Myerson's (1981) setting, the reader is referred to Section 2.6.1 for details. In the following we just briefly summarize the results. Again, buyers' valuation functions in this setting satisfy non-decreasing expected differences (see Definition 2.3). Thus, from Theorem 2.3 it follows that $f$ is Bayes-Nash incentive compatible if and only if it satisfies monotonicity. Using shortest path lengths to construct payments and picking $\underline{t}^{i}$ as the source node in $T_{f}^{i}$ for every buyer $i$ yields

$$
E_{-i}\left[P_{i}\left(r^{i}, t^{-i}\right)\right]=E_{-i}\left[v^{i}\left(f\left(r^{i}, t^{-i}\right) \mid r^{i}, t^{-i}\right)-v^{i}\left(f\left(\underline{t}^{i}, t^{-i}\right) \mid \underline{t}^{i}, t^{-i}\right)\right]-\int_{\underline{t}^{i}}^{r^{i}} q^{i}(s) d s .
$$

for our payment rule. Since buyers's type spaces are convex and their expected valuation functions are linear in their true types, we can apply Theorem 2.4. Hence, all Bayes-Nash incentive compatible 
allocation rules in this setting satisfy revenue equivalence. Based on (2.37) we again get

$$
U^{i}\left(r^{i} \mid r^{i}\right)=U^{i}\left(\underline{t}^{i} \mid \underline{t}^{i}\right)+\int_{\underline{t}^{i}}^{r^{i}} q^{i}(s) d s .
$$

as a necessary condition for Bayes-Nash incentive compatibility. 



\section{Monotonicity and Multi-Dimensional Types}

An allocation rule is called Bayes-Nash incentive compatible, if there exists a payment rule, such that truthful reports of agents' types form a Bayes-Nash equilibrium in the direct revelation mechanism consisting of the allocation rule and the payment rule. This chapter provides characterizations of Bayes-Nash incentive compatible allocation rules in social choice settings where agents' types are multi-dimensional and independently distributed. The agents have convex type spaces and quasi-linear utility functions. Their expected valuation functions are restricted to be convex in their own types. We allow for interdependent valuations. The characterizations are derived by constructing complete directed graphs on agents' type spaces with cost of manipulation as lengths of edges. Thus, the work presented in this chapter serves as the Bayes-Nash extension of a recent stream of literature that employs a network based approach to characterize dominant strategy incentive compatible allocation rules. It also extends the work of the foregoing chapter from one-dimensional to multi-dimensional types. We follow the same network approach as before and keep the structure of the chapter as close as possible to the foregoing one. This yields some overlap between both chapters but in this way Chapter 3 can be read as an independent entity.

Monotonicity of the allocation rule corresponds to the condition that all 2-cycles in the above mentioned graphs have non-negative length. In case agents' valuation functions satisfy non-decreasing expected differences, it is shown that monotonicity in conjunction with an integrability condition is a necessary and sufficient condition for Bayes-Nash incentive compatibility. Thus, compared to other characterization results that employ also conditions on agents' utility functions, we provide a characterization that only involves agents' valuations and the allocation rule. Our approach also allows us to work in a more general setup that allows for a broader class of valuation functions.

We show how corresponding payment rules are constructed for Bayes-Nash incentive compatible allocation rules by utilizing shortest path lengths in agents' graphs. We establish that revenue equivalence holds for all incentive compatible allocation rules in our setting. This result was already established by Krishna and Maenner (2001). However, our network approach yields an alternate proof of this result. 


\subsection{Introduction}

This chapter is concerned with the characterization of Bayes-Nash incentive compatible allocation rules in social choice settings where agents have independently distributed, multi-dimensional types and quasi-linear utility functions. That is, utility is the valuation of an allocation minus a payment. We allow for interdependent valuations across agents. Thus, an agent's valuation can, next to his own type, depend on the types of the other agents. We consider direct revelation mechanisms. A direct mechanism consists of an allocation rule and a payment rule. Based on a profile of type reports from all the agents, the allocation rule determines the outcome. In case of a combinatorial auction for example, the allocation rule determines which set of items each agent gets. Similarly, the payment rule determines what payment each agent has to make. We call the allocation rule BayesNash incentive compatible if there exists a payment rule such that truthful reporting of agents' types constitutes a Bayes-Nash equilibrium in the corresponding direct revelation mechanism consisting of the two rules.

The central task of this chapter is to extend the results of the foregoing chapter as far as possible to settings featuring multi-dimensional types. Thus, one major topic is the characterization of Bayes-Nash incentive compatible allocation rules. That means, for the social choice settings under consideration, we want to characterize the allocation rules for which we can guarantee the existence of a payment rule making them Bayes-Nash incentive compatible. In doing this we again aim for a framework that enables us to construct such payment rules. For example, consider once more the allocation rule which decides in a combinatorial auction for each set of bids which set of items each agent is winning. We want to be able to decide whether there exists a pricing scheme for winning bids that makes truthful bidding a Bayes-Nash equilibrium. If the answer is yes, we want to have the means to construct such a pricing scheme.

Hence, the other big topic addressed in this chapter is the construction of payment rules. We show how to build a payment rule that makes an allocation rule Bayes-Nash incentive compatible. In addition we show that, for the settings under consideration here, all payment rules making a particular allocation rule Bayes-Nash incentive compatible yield the same expected payments for the agents. 


\subsubsection{Related Work}

An allocation rule is dominant strategy incentive compatible, if there exists a payment rule such that for any report of the other agents an agent maximizes his own utility by reporting truthfully his type. Roberts (1979) implicitly uses a monotonicity condition on the allocation rule in order to derive his characterization of dominant strategy incentive compatible mechanisms in terms of affine maximizers for unrestricted preference domains. For a selection of restricted preference domains, Bikhchandani, Chatterji, Lavi, Mu'alem, Nisan and Sen (2006) characterize dominant strategy incentive compatibility directly in terms of a monotonicity condition on the allocation rule. Gui, Müller and Vohra (2004) extend these results to larger classes of preference domains by making a link to network theory. Saks and $\mathrm{Yu}(2005)$ go even further, showing that previous results extend to any convex multi-dimensional type space. One of the most recent representatives of this stream of literature is Monderer (2008).

The environment considered by Saks and Yu (2005) features quasi-linear utilities and multidimensional types. The allocation rule maps agents' type reports into a finite set of $m$ possible outcomes. An agent's type is a vector in $\mathbb{R}^{m}$ reflecting his valuation of the different possible outcomes. That is, the agent's valuation of some outcome $a$ is given by the $a^{\text {th }}$ element of his type vector. Agents' type spaces are assumed to be convex. Saks and Yu (2005) show that dominant strategy incentive compatible allocation rules in this setting can be characterized in terms of weak monotonicity. In order to derive this result they construct complete directed graphs in the following way: Take some agent and fix a profile of type reports for the others. Now, a directed graph is constructed by associating a node with each outcome and putting a directed edge between each ordered pair of nodes. Take two outcomes $a$ and $b$. Consider the difference of the valuation of $a$ and the valuation of $b$ with respect to every type for which truthfully reporting this type yields outcome $a$. The length of the network edge from $a$ to $b$ is defined as the infimum of all these differences. In this fashion a graph is constructed for every agent and every possible report profile of the other agents. Weak monotonicity states that for any two different outcomes $a$ and $b$, the sum of the two edge lengths from $a$ to $b$ and from $b$ to $a$ is non-negative.

Earlier, Rochet (1987) characterized dominant strategy implementation in cases where the set of outcomes is not necessarily finite. This assumption is crucial to the work of Saks and Yu (2005) and the others. He considers a setting where agents have multi-dimensional, convex type spaces and valuation functions which are linear with respect to their own true types. Making some additional differentiability assumptions, Rochet (1987) shows that in this case dominant strategy incentive compatibility can be characterized in terms of a monotonicity condition on the allocation rule plus 
an integrability condition.

Monotonicity has also been used to characterize Bayes-Nash incentive compatible allocation rules. Jehiel, Moldovanu and Stacchetti (1999) and Jehiel and Moldovanu (2001) develop characterizations for social choice settings where agents have multi-dimensional, convex type spaces and valuation functions which are linear with respect to their true types. In the first one agents' valuations are independent, whereas the second one allows for interdependent valuations. Both characterizations of Bayes-Nash incentive compatibility include a monotonicity condition on the allocation rule as well as an integrability condition comparable to the one presented by Rochet (1987) and a condition on agents' utility functions. The frameworks considered by Jehiel, Moldovanu and Stacchetti (1999) and Jehiel and Moldovanu (2001) can be folded into the environment considered by us in Müller, Perea and Wolf (2007b). There we also deal with social choice settings in which agents have multidimensional, convex type spaces and valuation functions which are linear in their true types. However, we allow for a broader class of possible interdependencies between agents' valuations. In order to establish our results in Müller, Perea and Wolf (2007b) we make a link to network theory. Archer and Kleinberg (2008) consider a single agent setting also featuring multi-dimensional types and linear valuation functions. For convex type spaces they characterize incentive compatibility in terms of a monotonicity and a local integrability condition of the allocation rule. They also deliver some insight for non-convex type spaces.

Krishna and Maenner (2001) consider a setting in which each agent has a convex type space and a valuation function that is convex in the agent's type. They show that in this setting revenue equivalence holds for all Bayes-Nash incentive compatible allocation rules. Heydenreich, Müller, Uetz and Vohra (2009) derive a characterization of revenue equivalence under more general conditions. Similar to Gui, Müller and Vohra (2004) and Saks and Yu (2005) they use networks to establish their results. For countable outcome spaces the aforementioned result of Krishna and Maenner (2001) follows as a consequence of their work.

\subsubsection{Our Contribution}

In Section 3.2 we state some basic assumptions and definitions. Throughout the chapter we assume that agents have quasi-linear utility functions and independently distributed, privately known, multidimensional types. Furthermore, we do not put any restrictions on the number of possible outcomes. We allow for interdependent valuations. However, we require that agents' expected valuation functions are convex in their own types. This framework is less restrictive than the one employed in 
Müller, Perea and Wolf (2007b) where linearity instead of convexity is required. On the other hand, it is more restrictive than the framework considered in the foregoing chapter for one-dimensional types. There the convexity of agents' types spaces and the convexity condition on agents' valuation functions are not needed for the characterization results. Nevertheless, many of the arguments that we employed in the analysis of the one-dimensional environment also go through in the multidimensional setting. Therefore we decided to keep the structure of this chapter as close as possible to the structure of Chapter 2, even though that yields some overlaps, especially in Section 3.3.

If an allocation rule is Bayes-Nash incentive compatible, then there exists a payment rule such that an agent's expected utility for truthfully reporting his type $t$ is at least as high as his expected utility for misreporting some type $s$. Similarly, an agent's expected utility for truthfully reporting type $s$ is at least as high as his expected utility for misreporting type $t$. By combining these two conditions we get a monotonicity condition on the allocation rule. This condition is the expected utility equivalent to the weak monotonicity condition utilized by Saks and Yu (2005) in the context of dominant strategy incentive compatible allocation rules. Monotonicity is a necessary condition for Bayes-Nash incentive compatibility. It expresses that the expected gain in valuation for truthfully reporting $t$ instead of misreporting $s$ should be at least as big as the expected gain in valuation for misreporting $t$ instead of truthfully reporting $s$.

Similar to the network approach of Gui, Müller and Vohra (2004) and Saks and Yu (2005) we construct graphs in Section 3.3. Recognizing that the constraints inherent in the definition of BayesNash incentive compatibility have a natural network interpretation we build complete directed graphs for agents' type spaces. To do so we associate a node with each type and put a directed edge between each ordered pair of nodes. The length of the edge going from the node associated with type $s$ to the node associated with the type $t$ is defined as the cost of manipulation. That is, the expected difference in an agent's valuation for truthfully reporting $s$ instead of misreporting $t$.

Different from the networks constructed by Gui, Müller and Vohra (2004) and Saks and Yu (2005) we construct only one graph for each agent. This is because we work in terms of expectations and do not consider each possible type profile of the other agents separately. Each of these graphs contains an infinite number of nodes as we associate a node with each possible type of an agent. One could also construct outcome based graphs, as done by Gui, Müller and Vohra (2004) and Saks and Yu (2005), by associating a node with each possible probability distribution over outcomes. However, these graphs possibly also contain an infinite number of nodes since we allow that the different possible type reports of an agent induce an infinite number of probability distributions over outcomes. For 
example, in the single item auction framework presented in Section 3.6.2 it is quite natural to allow that every different type report of an agent yields a different vector of expected conditional winning probabilities.

We show that an allocation rule is Bayes-Nash incentive compatible if and only if the networks described above contain no negative length cycles. Rochet (1987) shows that dominant strategy incentive compatibility can be characterized in terms of the absence of negative length cycles in similar graphs. Our result is the Bayes-Nash equivalent for his finding. Furthermore, we show as a lemma that the costs of manipulation are decomposition monotone if the allocation rule satisfies monotonicity and agents' valuation functions satisfy non-decreasing expected differences (definitions can be found in Section 3.2). The network construction and the derivation of these results are completely analogical to what we have done in the corresponding section of the foregoing chapter.

In chapter 2, dealing with one-dimensional types, we show for the case where the costs of manipulation are decomposition monotone that monotonicity is not only a necessary but also a sufficient condition for Bayes-Nash incentive compatibility. Unfortunately, once we turn to our multi-dimensional type setting, monotonicity alone is not sufficient anymore. Even with the additional convexity restrictions on agents' type spaces and valuation functions. This is illustrated by an example in Section 3.4. However, we are able to show that monotonicity in conjunction with an integrability condition is both necessary and sufficient. Using the lemma from the foregoing section it follows that monotonicity and the integrability condition are necessary and sufficient conditions for Bayes-Nash incentive compatibility in case that agents' valuation functions satisfy non-decreasing expected differences. Using examples it is illustrated that weak monotonicity and the integrability condition do not imply one another.

The main contribution of Section 3.4 is thus to derive for the setting described above a complete characterization of Bayes-Nash incentive compatibility in terms of monotonicity and an additional integrability condition. Thereby we achieve a characterization that depends purely on the valuations and the allocation rule. This characterization resembles the one derived by Rochet (1987) for dominant strategy incentive compatibility. However, our setting covers a broader class of valuation functions as Rochet (1987) requires them to be linear in the agents' own true types. The settings considered in Jehiel, Moldovanu and Stacchetti (1999), Jehiel and Moldovanu (2001) and Müller, Perea and Wolf (2007b) also all restrict agents' valuation functions to be linear in their own true types. These settings constitute special cases of the framework discussed in this chapter. How these settings can be folded into our framework is illustrated in Section 3.4.1 for Müller, Perea and Wolf 
(2007b) and in Section 3.6 for the other two.

If we know that an allocation rule is Bayes-Nash incentive compatible, we also want to know how to construct a corresponding payment rule. In Section 3.5 we show that such payment rules can be built by making use of shortest path lengths in the agents' networks. We also establish that in the environment considered by us, all Bayes-Nash incentive compatible allocation rules satisfy revenue equivalence. Thus, using our network approach, we can establish revenue equivalence under the same conditions as Krishna and Maenner (2001).

\subsection{Model \& Definitions}

In this chapter we consider a setting in which there is a set of possible outcomes $\Gamma$, as well as a set of agents $N=\{1, \ldots, n\}$. Each agent has some private information which is modeled by a multidimensional type. Specifically, each agent $i$ has a type $t^{i} \in T^{i}$ with $T^{i} \subseteq \mathbb{R}^{k}, k>1$. We assume that $T^{i}$ is convex for each agent $i$. The set of all complete type profiles $t=\left(t^{1}, \ldots, t^{n}\right)$ is denoted $T$, whereas the set of all type profiles excluding agent $i, t^{-i}=\left(t^{1}, \ldots, t^{i-1}, t^{i+1}, \ldots, t^{n}\right)$, is denoted $T^{-i}$. We assume that agents' types are independently distributed. Assuming that agent $i$ 's types are distributed according to some density $\pi^{i}$ on $T^{i}$, the joint density $\pi^{-i}$ on $T^{-i}$ is then given by

$$
\pi^{-i}\left(t^{-i}\right)=\prod_{\substack{j \in N \\ j \neq i}} \pi^{j}\left(t^{j}\right)
$$

We consider direct revelation mechanisms, consisting of a payment rule and an allocation rule. Based on a profile of reported types from all the agents, a payment rule

$$
P: T \mapsto \mathbb{R}^{n}
$$

assigns each agent with a payment. So, given a report profile $r^{-i}$ of the others, reporting type $r^{i}$ results in a payment $P_{i}\left(r^{i}, r^{-i}\right)$ for agent $i$. Similarly, an allocation rule

$$
f: T \mapsto \Gamma
$$

is mapping each profile of reported types into an outcome.

Suppose agent $i$ has true type $t^{i}$ and reports $r^{i}$ while the others have true types $t^{-i}$ and report $r^{-i}$. The value that agent $i$ assigns to the resulting outcome of the allocation rule is denoted by 
$v^{i}\left(f\left(r^{i}, r^{-i}\right) \mid t^{i}, t^{-i}\right)$. This setup allows for interdependent valuations across agents. That is, an agent's valuation for an outcome is not only influenced by his own type but also by the types of the other agents. Take for example an auction where several telephone companies compete for $3 \mathrm{G}$ spectrum licenses, see Kirchkamp and Moldovanu (2004). In this example a company's type reflects its cost structure as well as its expectation for future market demand based on a survey it took. This survey outcome is also of interest to the other telephone companies for their value assessments of the spectrum licenses. Thus, the companies' valuation functions are interdependent.

Agents' utility functions are quasi-linear. That is, an agent's utility is his valuation of an allocation minus his payment. Consider agent $i$ and assume that the other agents make their reports truthfully. If agent $i$ has true type $t^{i}$, then his expected utility for making a report $r^{i}$ is given by

$$
\begin{aligned}
U^{i}\left(r^{i} \mid t^{i}\right) & =\int_{T^{-i}}\left(v^{i}\left(f\left(r^{i}, t^{-i}\right) \mid t^{i}, t^{-i}\right)-P_{i}\left(r^{i}, t^{-i}\right)\right) \pi^{-i}\left(t^{-i}\right) d t^{-i} \\
& =E_{-i}\left[v^{i}\left(f\left(r^{i}, t^{-i}\right) \mid t^{i}, t^{-i}\right)-P_{i}\left(r^{i}, t^{-i}\right)\right] .
\end{aligned}
$$

We assume $E_{-i}\left[v^{i}\left(f\left(r^{i}, t^{-i}\right) \mid t^{i}, t^{-i}\right)\right]$ to be finite $\forall r^{i}, t^{i} \in T^{i}$. This holds for example if $\forall r^{i}, t^{i}$ the valuation function $v^{i}\left(f\left(r^{i}, t^{-i}\right) \mid t^{i}, t^{-i}\right)$, viewed as a function of $t^{-i}$, is bounded. In addition, we assume that agent $i$ 's expected valuation functions $E_{-i}\left[v^{i}\left(f\left(r^{i}, t^{-i}\right) \mid ., t^{-i}\right)\right]$ are convex in his true type. This is guaranteed if for example $v^{i}\left(f\left(r^{i}, t^{-i}\right) \mid ., t^{-i}\right)$ is convex for all $t^{-i}$ since the integral of a family of convex functions is convex.

Furthermore, we let $\partial E_{-i}\left[v^{i}\left(f\left(r^{i}, t^{-i}\right) \mid t^{i}, t^{-i}\right)\right]$ denote the set of all subgradients of $E_{-i}\left[v^{i}\left(f\left(r^{i}, t^{-i}\right) \mid ., t^{-i}\right)\right]$ at $t^{i}$. Note that $\partial E_{-i}\left[v^{i}\left(f\left(r^{i}, t^{-i}\right) \mid t^{i}, t^{-i}\right)\right]$ is a non-empty set for all $t^{i} \in T^{i}$ due to the convexity of $E_{-i}\left[v^{i}\left(f\left(r^{i}, t^{-i}\right) \mid ., t^{-i}\right)\right]$, see for example Rockafellar (1970), p.218. By $x^{i}$ we denote a selection from these subgradient sets. Specifically, for every $r^{i} \in T^{i}$ we pick an element of $\partial E_{-i}\left[v^{i}\left(f\left(r^{i}, t^{-i}\right) \mid r^{i}, t^{-i}\right)\right]$ which we denote by $x^{i}\left(r^{i}\right)$. That is,

$$
x^{i}\left(r^{i}\right) \in \partial E_{-i}\left[v^{i}\left(f\left(r^{i}, t^{-i}\right) \mid r^{i}, t^{-i}\right)\right] \quad \forall r^{i} \in T^{i} .
$$

Definition 3.1 (Bayes-Nash Incentive Compatibility) An allocation rule $f$ is Bayes-Nash incentive compatible if there exists a payment rule $P$ such that $\forall i \in N$ and $\forall r^{i}, \tilde{r}^{i} \in T^{i}$ :

$$
E_{-i}\left[v^{i}\left(f\left(r^{i}, t^{-i}\right) \mid r^{i}, t^{-i}\right)-P_{i}\left(r^{i}, t^{-i}\right)\right] \geq E_{-i}\left[v^{i}\left(f\left(\tilde{r}^{i}, t^{-i}\right) \mid r^{i}, t^{-i}\right)-P_{i}\left(\tilde{r}^{i}, t^{-i}\right)\right] .
$$


If an allocation rule $f$ is Bayes-Nash incentive compatible, we have also

$$
E_{-i}\left[v^{i}\left(f\left(\tilde{r}^{i}, t^{-i}\right) \mid \tilde{r}^{i}, t^{-i}\right)-P_{i}\left(\tilde{r}^{i}, t^{-i}\right)\right] \geq E_{-i}\left[v^{i}\left(f\left(r^{i}, t^{-i}\right) \mid \tilde{r}^{i}, t^{-i}\right)-P_{i}\left(r^{i}, t^{-i}\right)\right]
$$

By adding (3.3) and (3.4) we get

$$
\begin{aligned}
& E_{-i}\left[v^{i}\left(f\left(r^{i}, t^{-i}\right) \mid r^{i}, t^{-i}\right)+v^{i}\left(f\left(\tilde{r}^{i}, t^{-i}\right) \mid \tilde{r}^{i}, t^{-i}\right)-P_{i}\left(r^{i}, t^{-i}\right)-P_{i}\left(\tilde{r}^{i}, t^{-i}\right)\right] \\
\geq & E_{-i}\left[v^{i}\left(f\left(\tilde{r}^{i}, t^{-i}\right) \mid r^{i}, t^{-i}\right)+v^{i}\left(f\left(r^{i}, t^{-i}\right) \mid \tilde{r}^{i}, t^{-i}\right)-P_{i}\left(r^{i}, t^{-i}\right)-P_{i}\left(\tilde{r}^{i}, t^{-i}\right)\right] .
\end{aligned}
$$

Note that agent $i$ 's expected payments for reporting types $r^{i}$ and $\tilde{r}^{i}$ appear on both sides of the above inequality and can be canceled. This however is only possible because we work under the assumption of independently distributed types. Dropping this assumption would result in the following: Because of stemming from (3.3), the expected payment for reporting $r^{i}$ on the left-hand side of (3.5) would be conditional on agent $i$ having true type $r^{i}$. On the other hand, the expected payment for reporting $r^{i}$ on the right-hand side of (3.5) would be conditional on agent $i$ having true type $\tilde{r}^{i}$ since it stems from (3.4). Thus, the two expected payments could not be canceled anymore.

Canceling the expected payments and rearranging the terms in (3.5) yields the following monotonicity condition:

Definition 3.2 (Monotonicity) An allocation rule $f$ satisfies monotonicity if $\forall i \in N$ and $\forall r^{i}, \tilde{r}^{i} \in$ $T^{i}$ :

$$
\begin{aligned}
& E_{-i}\left[v^{i}\left(f\left(r^{i}, t^{-i}\right) \mid r^{i}, t^{-i}\right)-v^{i}\left(f\left(\tilde{r}^{i}, t^{-i}\right) \mid r^{i}, t^{-i}\right)\right] \\
\geq & E_{-i}\left[v^{i}\left(f\left(r^{i}, t^{-i}\right) \mid \tilde{r}^{i}, t^{-i}\right)-v^{i}\left(f\left(\tilde{r}^{i}, t^{-i}\right) \mid \tilde{r}^{i}, t^{-i}\right)\right] .
\end{aligned}
$$

The monotonicity condition above is the expected utility equivalent to the 2-cycle inequality of Gui, Müller and Vohra (2004), the weak monotonicity (W-Mon) condition of Bikhchandani, Chatterji, Lavi, Mu'alem, Nisan and Sen (2006) and the weak monotonicity condition of Saks and Yu (2005). It is the same as the condition termed weak monotonicity in Müller, Perea and Wolf (2007b). The rationale for naming this condition monotonicity becomes evident once one considers valuation functions that are linear with respect to agents' types, see for example Section 3.4.1 or Section 3.6 of this chapter. Based on the above derivation it is clear that monotonicity is a necessary condition for Bayes-Nash incentive compatibility. In Section 3.4 we discuss to what extend monotonicity also 
serves as a sufficient condition.

Furthermore, let us introduce at this point the following condition for agents' valuation functions:

Definition 3.3 (Non-decreasing Expected Differences) Consider $r^{i}, \tilde{r}^{i}, t^{i}, \tilde{t}^{i} \in T^{i}$ such that

$$
\begin{aligned}
& E_{-i}\left[v^{i}\left(f\left(r^{i}, t^{-i}\right) \mid t^{i}, t^{-i}\right)-v^{i}\left(f\left(\tilde{r}^{i}, t^{-i}\right) \mid t^{i}, t^{-i}\right)\right] \\
\geq & E_{-i}\left[v^{i}\left(f\left(r^{i}, t^{-i}\right) \mid \tilde{t}^{i}, t^{-i}\right)-v^{i}\left(f\left(\tilde{r}^{i}, t^{-i}\right) \mid \tilde{t}^{i}, t^{-i}\right)\right] .
\end{aligned}
$$

The valuation function satisfies non-decreasing expected differences if $\forall \bar{t}^{i} \in T^{i}$ s.t. $\bar{t}^{i}=(1-\alpha) \tilde{t}^{i}+\alpha t^{i}, \alpha>1$ we have

$$
\begin{aligned}
& E_{-i}\left[v^{i}\left(f\left(r^{i}, t^{-i}\right) \mid \bar{t}^{i}, t^{-i}\right)-v^{i}\left(f\left(\tilde{r}^{i}, t^{-i}\right) \mid \bar{t}^{i}, t^{-i}\right)\right] \\
\geq & E_{-i}\left[v^{i}\left(f\left(r^{i}, t^{-i}\right) \mid t^{i}, t^{-i}\right)-v^{i}\left(f\left(\tilde{r}^{i}, t^{-i}\right) \mid t^{i}, t^{-i}\right)\right] .
\end{aligned}
$$

The above condition deals with the marginal change in expected valuation with respect to the reported type. Consider the change in expected valuation for making a report $r^{i}$ instead of $\tilde{r}^{i}$. Assume that there exist types $t^{i}$ and $\tilde{t}^{i}$ such that this change is larger or at least as large if the agent has true type $t^{i}$ instead of $\tilde{t}^{i}$. This is illustrated in Figure 3.1 which depicts the differences in expected valuation along the line through types $\tilde{t}^{i}$ and $t^{i}$. Now consider the agent having a true type which is even further away from $\tilde{t}^{i}$ than $t^{i}$ (in the direction of $t^{i}$ ). The condition then requires that the change in expected valuation is at least as large as in the case where the agent has true type $t^{i}$. That is, the point corresponding to this change in expected valuation in Figure 3.1 has to lie in the shaded area. Non-decreasing expected differences is a slightly weaker condition than increasing differences. The later requires that the marginal change in valuation with respect to the allocation is strictly increasing in the type. Specifically, it requires that that for all $r^{i} \geq \hat{r}^{i}, r^{i} \neq \hat{r}^{i}$ and all $t^{i} \geq \hat{t}^{i}, t^{i} \neq \hat{t}^{i}$ in $T^{i}$,

$$
\begin{aligned}
& E_{-i}\left[v^{i}\left(f\left(r^{i}, t^{-i}\right) \mid t^{i}, t^{-i}\right)-v^{i}\left(f\left(\hat{r}^{i}, t^{-i}\right) \mid t^{i}, t^{-i}\right)\right] \\
> & E_{-i}\left[v^{i}\left(f\left(r^{i}, t^{-i}\right) \mid \hat{t}^{i}, t^{-i}\right)-v^{i}\left(f\left(\hat{r}^{i}, t^{-i}\right) \mid \hat{t}^{i}, t^{-i}\right)\right] .
\end{aligned}
$$

Note that we assume agent $i$ 's expected valuation functions $E_{-i}\left[v^{i}\left(f\left(r^{i}, t^{-i}\right) \mid ., t^{-i}\right)\right]$ to be convex in his true type. As illustrated in Figures 3.2 and 3.3, this convexity does not imply nondecreasing expected differences and vice versa. Both figures depict expected valuation functions for reporting $r^{i}$ and $\tilde{r}^{i}$ along a line in agent $i$ 's type space. In Figure 3.2, $E_{-i}\left[v^{i}\left(f\left(r^{i}, t^{-i}\right) \mid ., t^{-i}\right)\right]$ 


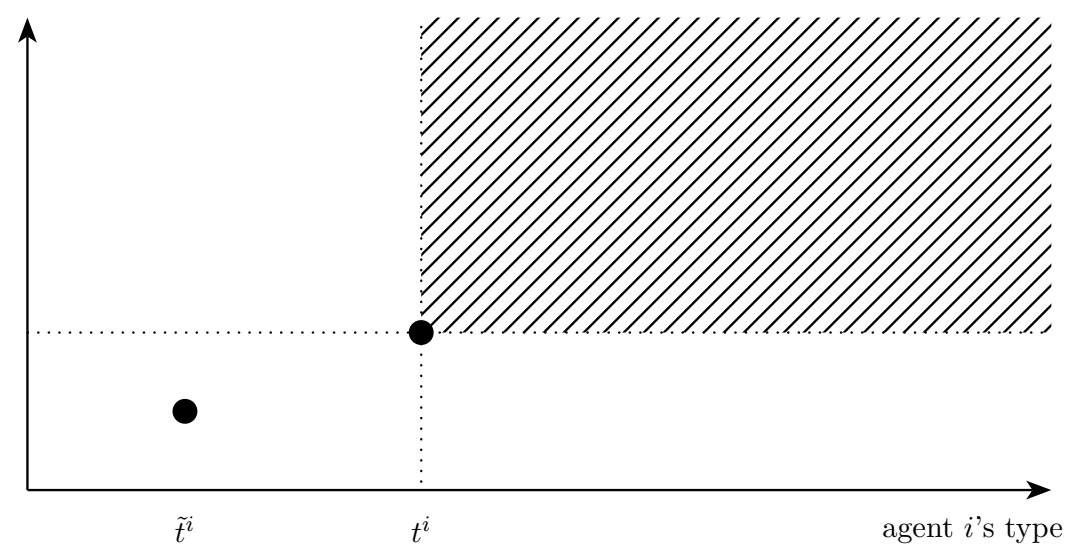

Figure 3.1: Illustration non-decreasing expected differences: The change in expected valuation for making a report $r^{i}$ instead of $\tilde{r}^{i}, E_{-i}\left[v^{i}\left(f\left(r^{i}, t^{-i}\right) \mid ., t^{-i}\right)-v^{i}\left(f\left(\tilde{r}^{i}, t^{-i}\right) \mid ., t^{-i}\right)\right]$, is depicted along the $y$-axis.

and $E_{-i}\left[v^{i}\left(f\left(\tilde{r}^{i}, t^{-i}\right) \mid ., t^{-i}\right)\right]$ are convex but the non-decreasing expected differences condition is clearly violated. In Figure 3.3 on the other hand, non-decreasing expected differences is satisfied

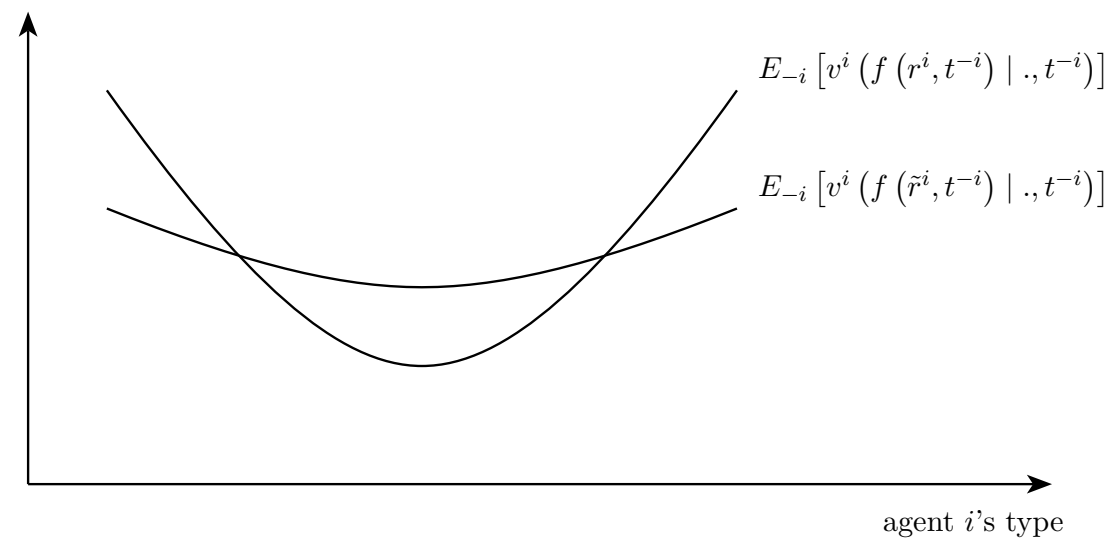

Figure 3.2: Convexity does not imply non-decreasing expected differences.

but $E_{-i}\left[v^{i}\left(f\left(r^{i}, t^{-i}\right) \mid ., t^{-i}\right)\right]$ and $E_{-i}\left[v^{i}\left(f\left(\tilde{r}^{i}, t^{-i}\right) \mid ., t^{-i}\right)\right]$ are not convex. Finally, note that an agent's valuation function which is linear in his true type satisfies the non-decreasing expected differences condition. Such linear settings are discussed in Section 3.4.1 and Section 3.6. 


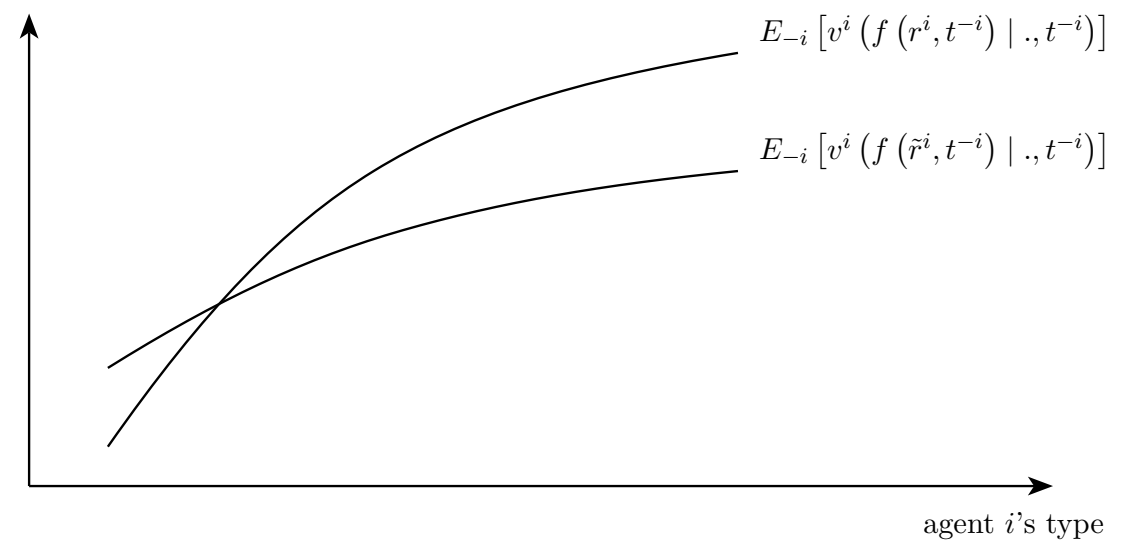

Figure 3.3: Non-decreasing expected differences does not imply convexity.

\subsection{A Network Interpretation}

Note that the content of this section corresponds to Section 2.3 in the foregoing chapter. It is repeated in order to enhance the readability of this chapter as a single, stand alone entity. Readers who already familiarized themselves with the content of Section 2.3 are invited to skip ahead to the next section.

Let us begin by briefly reviewing a well-known result from the field of network flow theory. ${ }^{1}$ Let $X=\left\{x_{1}, \ldots, x_{k}\right\}$ be a finite set of variables. Consider the following system of constraints:

$$
x_{i}-x_{j} \leq w_{i j} \quad \forall i, j \in\{1, \ldots, k\},
$$

where $w_{i j}$ is some constant specific to the ordered pair $(i, j)$. The system can be associated with a network by constructing a directed, weighted graph whose nodes correspond to the variables. A directed edge is put between each ordered pair of nodes. The length of the edge from the node corresponding to $x_{i}$ to the node corresponding to $x_{j}$ is given by $w_{i j}$.

It is a well-known result (see for example Shostak (1981)) that there is no negative length cycle in the associated network if and only if the system of linear inequalities in (3.6) is feasible. The system being feasible means that there exists an assignment of real values to the variables such that the constraints in (3.6) are satisfied. Furthermore, if the system is feasible then one feasible solution is to assign to each $x_{i}$ the length of a shortest path from the node associated with $x_{i}$ to some arbitrary

\footnotetext{
${ }^{1}$ A comprehensive introduction to network flows is given in Ahuja, Magnanti and Orlin (1993).
} 
source node. ${ }^{2}$

In order to see that the constraints in (3.3) have a natural network interpretation it is useful to rewrite (3.3) as follows:

$$
E_{-i}\left[P_{i}\left(r^{i}, t^{-i}\right)-P_{i}\left(\tilde{r}^{i}, t^{-i}\right)\right] \leq E_{-i}\left[v^{i}\left(f\left(r^{i}, t^{-i}\right) \mid r^{i}, t^{-i}\right)-v^{i}\left(f\left(\tilde{r}^{i}, t^{-i}\right) \mid r^{i}, t^{-i}\right)\right] .
$$

Considering a specific allocation rule, the right-hand side of (3.7) is a constant. Thus, we have a system of difference constraints as described in (3.6) (except that we are now dealing with an infinite number of variables and constraints).

Given this observation, we associate the system of inequalities (3.7) with a network in the same way as is described above. For each agent we build a complete directed graph $T_{f}^{i}$. A node is associated with each type and a directed edge is put between each ordered pair of nodes. For agent $i$ the length of an edge directed from $r^{i}$ to $\tilde{r}^{i}$ is denoted $l^{i}\left(r^{i}, \tilde{r}^{i}\right)$ and is defined as the cost of manipulation:

$$
l^{i}\left(r^{i}, \tilde{r}^{i}\right)=E_{-i}\left[v^{i}\left(f\left(r^{i}, t^{-i}\right) \mid r^{i}, t^{-i}\right)-v^{i}\left(f\left(\tilde{r}^{i}, t^{-i}\right) \mid r^{i}, t^{-i}\right)\right]
$$

Given our previous assumptions, the edge length is finite. For technical reasons we allow for loops. However, note that an edge directed from $r^{i}$ to $r^{i}$ has length $l^{i}\left(r^{i}, r^{i}\right)=0$.

Using the above definition of the edge lengths, the monotonicity condition can be written as

$$
l^{i}\left(r^{i}, \tilde{r}^{i}\right)+l^{i}\left(\tilde{r}^{i}, r^{i}\right) \geq 0 \quad \forall i \in N, \forall r^{i}, \tilde{r}^{i} \in T^{i}
$$

So monotonicity corresponds to the absence of negative length 2-cycles in the agents' graphs described above.

Rochet (1987) observed that dominant strategy incentive compatibility can be characterized in terms of the absence of negative length cycles in similar graphs. Using the same proof technique, we can derive such a characterization for Bayes-Nash incentive compatibility as well.

Theorem 3.1 An allocation rule $f$ is Bayes-Nash incentive compatible if and only if there is no negative length cycle in $T_{f}^{i}, \forall i \in N$.

\footnotetext{
${ }^{2}$ In order to be consistent with the existing literature we defined the system of constraints as in (3.6). However, in network theory the constraints are commonly defined as $x_{j}-x_{i} \leq w_{i j}$. In this case, if the system is feasible then one feasible solution is to assign to each $x_{i}$ the length of a shortest path from some arbitrary source node to the node associated with $x_{i}$.
} 


\section{Proof (Adapted from Rochet (1987).)}

Take some agent $i$ and let $C=\left(r_{1}^{i}, \ldots, r_{m}^{i}, r_{m+1}^{i}=r_{1}^{i}\right)$ denote a cycle in $T_{f}^{i}$. Let us assume that $f$ is Bayes-Nash incentive compatible. Using (3.7) and the edge length definition (3.8), this implies that for every $j \in\{1, \ldots, m\}$,

$$
E_{-i}\left[P_{i}\left(r_{j}^{i}, t^{-i}\right)-P_{i}\left(r_{j+1}^{i}, t^{-i}\right)\right] \leq l^{i}\left(r_{j}^{i}, r_{j+1}^{i}\right)
$$

Adding up these inequalities yields

$$
0 \leq \sum_{j=1}^{m} l^{i}\left(r_{j}^{i}, r_{j+1}^{i}\right),
$$

so cycle $C$ has non-negative length.

Conversely, let us assume that there exists no negative length cycle in $T_{f}^{i}, \forall i \in N$. For each agent $i$ we pick an arbitrary source node $r_{0}^{i} \in T^{i}$ and define $\forall r^{i} \in T^{i}$

$$
p^{i}\left(r^{i}\right)=\inf \sum_{j=1}^{m} l^{i}\left(r_{j}^{i}, r_{j+1}^{i}\right),
$$

where the infimum is taken over all paths $A=\left(r_{1}^{i}=r^{i}, \ldots, r_{m+1}^{i}=r_{0}^{i}\right)$ in $T_{f}^{i}$, that is, all paths that start at $r^{i}$ and end at $r_{0}^{i}$. Absence of negative length cycles implies that $p^{i}\left(r_{0}^{i}\right)=0$. Furthermore, $\forall r^{i} \in T^{i}$ we have

$$
p^{i}\left(r_{0}^{i}\right) \leq p^{i}\left(r^{i}\right)+l^{i}\left(r_{0}^{i}, r^{i}\right)
$$

which implies that $p^{i}\left(r^{i}\right)$ is finite. For every pair $r^{i}, \tilde{r}^{i} \in T^{i}$ we also have

$$
p^{i}\left(r^{i}\right) \leq p^{i}\left(\tilde{r}^{i}\right)+l^{i}\left(r^{i}, \tilde{r}^{i}\right)
$$

Thus, by setting

$$
P_{i}\left(r^{i}, t^{-i}\right)=p^{i}\left(r^{i}\right) \quad \forall t^{-i} \in T^{-i}
$$

and using (3.8) we get

$$
E_{-i}\left[P_{i}\left(r^{i}, t^{-i}\right)-P_{i}\left(\tilde{r}^{i}, t^{-i}\right)\right] \leq E_{-i}\left[v^{i}\left(f\left(r^{i}, t^{-i}\right) \mid r^{i}, t^{-i}\right)-v^{i}\left(f\left(\tilde{r}^{i}, t^{-i}\right) \mid r^{i}, t^{-i}\right)\right]
$$

Hence, the constraints in (3.7) are satisfied and $f$ is Bayes-Nash incentive compatible. Note that it is actually sufficient to pick a payment rule such that $E_{-i}\left[P_{i}\left(r^{i}, t^{-i}\right)\right]=p^{i}\left(r^{i}\right)+c^{i}$. This allows for a variety of payment rules yielding the same expected payments up to an additive constant. 
We conclude this section with a condition for the costs of manipulation that is used in the derivation of the characterization theorems presented in the following section.

Definition 3.4 (Decomposition Monotonicity) The costs of manipulation are decomposition monotone if $\forall \underline{r}^{i}, \bar{r}^{i} \in T^{i}$ and $\forall r^{i} \in T^{i}$ s.t. $r^{i}=(1-\alpha) \underline{r}^{i}+\alpha \bar{r}^{i}, \alpha \in(0,1)$ we have

$$
l^{i}\left(\underline{r}^{i}, \bar{r}^{i}\right) \geq l^{i}\left(\underline{r}^{i}, r^{i}\right)+l^{i}\left(r^{i}, \bar{r}^{i}\right) .
$$

So considering a pair of nodes, if decomposition monotonicity holds then the direct edge between those nodes is at least as long as any path connecting the same two nodes via nodes lying on the line segment between them. An illustrative example is given in Figure 3.4. Decomposition monotonicity implies that the edge from $\underline{r}^{i}$ to $\bar{r}^{i}$ is at least as long as the path $A=\left(\underline{r}^{i}, r_{* *}^{i}, \bar{r}^{i}\right)$ and that $A$ is at least as long as the path $\tilde{A}=\left(\underline{r}^{i}, r_{*}^{i}, r_{* *}^{i}, r_{* * *}^{i}, \bar{r}^{i}\right)$.

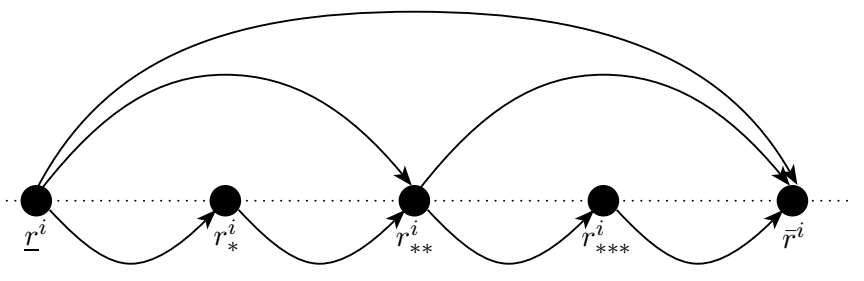

Figure 3.4: Example decomposition monotonicity.

Monotonicity and non-decreasing expected differences together imply that the costs of manipulation are decomposition monotone:

Lemma 3.1 If $f$ satisfies monotonicity and the valuation function satisfies non-decreasing expected differences then the costs of manipulation are decomposition monotone.

\section{Proof}

Take some agent $i$ and let $\underline{r}^{i}, \bar{r}^{i} \in T^{i}$. Let $r^{i} \in T^{i}$ such that $r^{i}=(1-\alpha) \underline{r}^{i}+\alpha \bar{r}^{i}$ for some $\alpha \in(0,1)$. Monotonicity implies that

$$
\begin{aligned}
& E_{-i}\left[v^{i}\left(f\left(r^{i}, t^{-i}\right) \mid r^{i}, t^{-i}\right)-v^{i}\left(f\left(\bar{r}^{i}, t^{-i}\right) \mid r^{i}, t^{-i}\right)\right] \\
\geq & E_{-i}\left[v^{i}\left(f\left(r^{i}, t^{-i}\right) \mid \bar{r}^{i}, t^{-i}\right)-v^{i}\left(f\left(\bar{r}^{i}, t^{-i}\right) \mid \bar{r}^{i}, t^{-i}\right)\right] .
\end{aligned}
$$


Since the valuation function satisfies non-decreasing expected differences we have

$$
\begin{aligned}
& E_{-i}\left[v^{i}\left(f\left(r^{i}, t^{-i}\right) \mid \underline{r}^{i}, t^{-i}\right)-v^{i}\left(f\left(\bar{r}^{i}, t^{-i}\right) \mid \underline{r}^{i}, t^{-i}\right)\right] \\
\geq & E_{-i}\left[v^{i}\left(f\left(r^{i}, t^{-i}\right) \mid r^{i}, t^{-i}\right)-v^{i}\left(f\left(\bar{r}^{i}, t^{-i}\right) \mid r^{i}, t^{-i}\right)\right] .
\end{aligned}
$$

Adding $E_{-i}\left[v^{i}\left(f\left(\underline{r}^{i}, t^{-i}\right) \mid \underline{r}^{i}, t^{-i}\right)-v^{i}\left(f\left(r^{i}, t^{-i}\right) \mid \underline{r}^{i}, t^{-i}\right)\right]$ on both sides of the later inequality yields

$$
\begin{aligned}
& E_{-i}\left[v^{i}\left(f\left(r^{i}, t^{-i}\right) \mid \underline{r}^{i}, t^{-i}\right)-v^{i}\left(f\left(\bar{r}^{i}, t^{-i}\right) \mid \underline{r}^{i}, t^{-i}\right)\right] \\
& +E_{-i}\left[v^{i}\left(f\left(\underline{r}^{i}, t^{-i}\right) \mid \underline{r}^{i}, t^{-i}\right)-v^{i}\left(f\left(r^{i}, t^{-i}\right) \mid \underline{r}^{i}, t^{-i}\right)\right] \\
\geq & E_{-i}\left[v^{i}\left(f\left(r^{i}, t^{-i}\right) \mid r^{i}, t^{-i}\right)-v^{i}\left(f\left(\bar{r}^{i}, t^{-i}\right) \mid r^{i}, t^{-i}\right)\right] \\
& +E_{-i}\left[v^{i}\left(f\left(\underline{r}^{i}, t^{-i}\right) \mid \underline{r}^{i}, t^{-i}\right)-v^{i}\left(f\left(r^{i}, t^{-i}\right) \mid \underline{r}^{i}, t^{-i}\right)\right] .
\end{aligned}
$$

Notice that the first and the last term on the left-hand side of the inequality cancel. Hence

$$
\begin{aligned}
& E_{-i}\left[v^{i}\left(f\left(\underline{r}^{i}, t^{-i}\right) \mid \underline{r}^{i}, t^{-i}\right)-v^{i}\left(f\left(\bar{r}^{i}, t^{-i}\right) \mid \underline{r}^{i}, t^{-i}\right)\right] \\
\geq & E_{-i}\left[v^{i}\left(f\left(\underline{r}^{i}, t^{-i}\right) \mid \underline{r}^{i}, t^{-i}\right)-v^{i}\left(f\left(r^{i}, t^{-i}\right) \mid \underline{r}^{i}, t^{-i}\right)\right] \\
& +E_{-i}\left[v^{i}\left(f\left(r^{i}, t^{-i}\right) \mid r^{i}, t^{-i}\right)-v^{i}\left(f\left(\bar{r}^{i}, t^{-i}\right) \mid r^{i}, t^{-i}\right)\right] .
\end{aligned}
$$

Using (3.8) this can be written as

$$
l^{i}\left(\underline{r}^{i}, \bar{r}^{i}\right) \geq l^{i}\left(\underline{r}^{i}, r^{i}\right)+l^{i}\left(r^{i}, \bar{r}^{i}\right)
$$

so the costs of manipulation are decomposition monotone.

\subsection{Sufficiency of Monotonicity \& Path Independence}

As mentioned in Section 3.2, monotonicity is a necessary condition for Bayes-Nash incentive compatibility. In Chapter 2 we have shown that if agents' type spaces are one-dimensional then monotonicity serves as a sufficient condition for Bayes-Nash incentive compatibility. Unfortunately, if type spaces are multi-dimensional then monotonicity alone no longer suffices. This is illustrated below in Example 3.1 . 
This example is constructed based on the following insight. Suppose that agent $i$ 's expected valuations have a very simple linear form. Specifically, let us assume that

$$
E_{-i}\left[v^{i}\left(f\left(r^{i}, t^{-i}\right) \mid t^{i}, t^{-i}\right)\right]=r^{i} B_{i} t^{i \prime}
$$

where $B_{i}$ is some agent specific $k \times k$ matrix and ' denotes the transpose.

Monotonicity requires that

$$
\left(r^{i}-\tilde{r}^{i}\right) B_{i}\left(r^{i}-\tilde{r}^{i}\right)^{\prime} \geq 0 \quad \forall r^{i}, \tilde{r}^{i} \in T^{i}
$$

Note that

$$
B_{i}=\frac{1}{2}\left(B_{i}+B_{i}^{\prime}\right)+\frac{1}{2}\left(B_{i}-B_{i}^{\prime}\right) .
$$

That is, $B_{i}$ can be decomposed into a symmetric part $\frac{1}{2}\left(B_{i}+B_{i}^{\prime}\right)$ and an anti-symmetric part $\frac{1}{2}\left(B_{i}-B_{i}^{\prime}\right)$. Monotonicity is already satisfied if the symmetric part of $B_{i}$ is positive semi-definite. However, there are no negative length cycles in $T_{f}^{i}$ (and thus $f$ is Bayes-Nash incentive compatible) if and only if $B_{i}$ is symmetric and positive semi-definite. Both results follow from Rockafellar (1970), p. 240 .

Example 3.1 For simplicity we assume that there exists only a single agent. The agent's type is one of three extreme types, denoted $x, y$ and $z$, or any convex combination of these. His type space can be parameterized by a simplex with vertices $x=(1,0,0), y=(0,1,0)$ and $z=(0,0,1)$. Thus, the agent's type space $T=\operatorname{conv}\{x, y, z\}$ consists of the convex hull of the three unit vectors in $\mathbb{R}^{3}$.

There are three elementary outcomes, denoted $a, b$ and $c$. If the agent is of type $x$, his valuations for these outcomes are given by the first column of the following matrix

$$
V=\left(\begin{array}{lll}
2 & 0 & 3 \\
3 & 2 & 0 \\
0 & 3 & 2
\end{array}\right) .
$$

The first element in this column is his valuation for $a$, the second one for $b$ and the third one for $c$. Similarly, if the agent is of type $y$ or $z$, his valuations for the elementary outcomes are given by the second and the third column of $V$.

The allocation rule $f$ is a linear mapping associating each type report with a probability distribution over the three elementary outcomes. The outcome space $\Gamma$ is the set of all possible probability 
distributions on $\{a, b, c\}$. Generic element $\gamma=\left(\gamma_{a}, \gamma_{b}, \gamma_{c}\right)$ indicates that a is achieved with probability $\gamma_{a}, b$ with probability $\gamma_{b}$ and $c$ with probability $\gamma_{c}$. The allocation rule works as follows: if the agent reports $x$ as his type then $f$ awards him with the second-best outcome according to this type, that is $f(x)=(1,0,0)$. Similarly, $f(y)=(0,1,0)$ and $f(z)=(0,0,1)$. In general we have $f(r)=r I$, where $I$ denotes the $3 \times 3$ identity matrix.

The agent's valuation function is $v(f(r) \mid t)=f(r) V t^{\prime}=r V t^{\prime}$. As easily can be checked (by verifying that the symmetric part $\frac{1}{2}\left(V+V^{\prime}\right)$ of $V$ is positive definite), monotonicity is satisfied. That is,

$$
(r-\tilde{r}) V(r-\tilde{r})^{\prime} \geq 0 \quad \forall r, \tilde{r} \in T .
$$

Nevertheless, the 3-cycle $C=(x, y, z, x)$ has length $l(x, y)+l(y, z)+l(z, x)=-3$ (see also Figure 3.5). The existence of such a negative length cycle implies that $f$ is not Bayes-Nash incentive compatible (see Theorem 3.1).

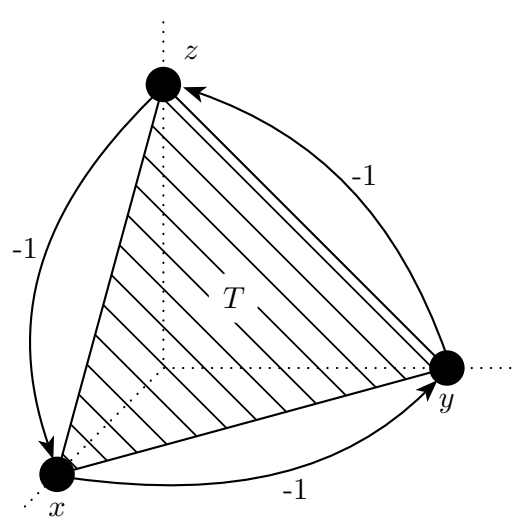

Figure 3.5: The negative cycle $C$ in Example 3.1.

From the above example it is evident that monotonicity alone is not enough to ensure BayesNash incentive compatibility. However, in the following we are going to show that monotonicity together with an integrability condition is sufficient if decomposition monotonicity (see Definition $3.4)$ is satisfied. 
Definition 3.5 (Path Independence) Let $\psi: T^{i} \mapsto \mathbb{R}^{k}$ be a vector field. We call $\psi$ path independent if for any two $\underline{r}^{i}, \bar{r}^{i} \in T^{i}$ the path integral of $\psi$ from $\underline{r}^{i}$ to $\bar{r}^{i}$

$$
\int_{\underline{r}^{i}, S}^{\bar{r}^{i}} \psi
$$

is independent of the path of integration $S$.

Recall that in Section 3.2, see (3.2), we defined

$$
x^{i}\left(r^{i}\right) \in \partial E_{-i}\left[v^{i}\left(f\left(r^{i}, t^{-i}\right) \mid r^{i}, t^{-i}\right)\right] \quad \forall r^{i} \in T^{i} .
$$

So $x^{i}$ is a vector field $T^{i} \mapsto \mathbb{R}^{k}$.

Theorem 3.2 Suppose that every agent $i$ has a convex type space and expected valuation functions $E_{-i}\left[v^{i}\left(f\left(r^{i}, t^{-i}\right) \mid ., t^{-i}\right)\right]$ which are convex in his true type. Also, suppose that the allocation rule $f$ and agents' valuation functions are such that the costs of manipulation satisfy decomposition monotonicity. Then the following statements are equivalent:

1) $f$ is Bayes-Nash incentive compatible.

2) $f$ satisfies monotonicity and for every agent $i, x^{i}$ is path independent.

\section{Proof}

$(\mathbf{1}) \Rightarrow(\mathbf{2})$ : Let us assume that $f$ is Bayes-Nash incentive compatible. As mentioned in Section 3.2, the necessity of monotonicity follows trivially. Furthermore, from Theorem 3.1 it follows that for every agent $i$ the graph $T_{f}^{i}$ has no negative length cycles. Let $C=\left(r_{1}^{i}, \ldots, r_{m}^{i}, r_{m+1}^{i}=r_{1}^{i}\right)$ denote a cycle in $T_{f}^{i}$. Absence of negative length cycles implies that

$$
\sum_{j=1}^{m} l^{i}\left(r_{j}^{i}, r_{j+1}^{i}\right) \geq 0 .
$$

Note that

$$
\begin{aligned}
\sum_{j=1}^{m} l^{i}\left(r_{j}^{i}, r_{j+1}^{i}\right) & =\sum_{j=1}^{m} E_{-i}\left[v^{i}\left(f\left(r_{j}^{i}, t^{-i}\right) \mid r_{j}^{i}, t^{-i}\right)-v^{i}\left(f\left(r_{j+1}^{i}, t^{-i}\right) \mid r_{j}^{i}, t^{-i}\right)\right] \\
& =\sum_{j=1}^{m} E_{-i}\left[v^{i}\left(f\left(r_{j+1}^{i}, t^{-i}\right) \mid r_{j+1}^{i}, t^{-i}\right)-v^{i}\left(f\left(r_{j+1}^{i}, t^{-i}\right) \mid r_{j}^{i}, t^{-i}\right)\right]
\end{aligned}
$$

where the first equality follows from (3.8) and the second equality stems from rearranging the terms 
of the summation. By definition of the subgradient we have for any $r_{j}^{i}, r_{j+1}^{i}$ in $C$ that

$$
E_{-i}\left[v^{i}\left(f\left(r_{j+1}^{i}, t^{-i}\right) \mid r_{j}^{i}, t^{-i}\right)-v^{i}\left(f\left(r_{j+1}^{i}, t^{-i}\right) \mid r_{j+1}^{i}, t^{-i}\right)\right] \geq\left(r_{j}^{i}-r_{j+1}^{i}\right) x^{i}\left(r_{j+1}^{i}\right) .
$$

Multiplying both sides by -1 yields

$$
E_{-i}\left[v^{i}\left(f\left(r_{j+1}^{i}, t^{-i}\right) \mid r_{j+1}^{i}, t^{-i}\right)-v^{i}\left(f\left(r_{j+1}^{i}, t^{-i}\right) \mid r_{j}^{i}, t^{-i}\right)\right] \leq\left(r_{j+1}^{i}-r_{j}^{i}\right) x^{i}\left(r_{j+1}^{i}\right)
$$

which implies that

$$
\sum_{j=1}^{m} E_{-i}\left[v^{i}\left(f\left(r_{j+1}^{i}, t^{-i}\right) \mid r_{j+1}^{i}, t^{-i}\right)-v^{i}\left(f\left(r_{j+1}^{i}, t^{-i}\right) \mid r_{j}^{i}, t^{-i}\right)\right] \leq \sum_{j=1}^{m}\left(r_{j+1}^{i}-r_{j}^{i}\right) x^{i}\left(r_{j+1}^{i}\right) .
$$

Together with the absence of negative length cycles this yields

$$
\sum_{j=1}^{m}\left(r_{j+1}^{i}-r_{j}^{i}\right) x^{i}\left(r_{j+1}^{i}\right) \geq 0
$$

Thus, $x^{i}$ is cyclically monotone. ${ }^{3}$ From Rockafellar (1970), Theorem 24.8, it follows that there exists a convex function $\varphi: T^{i} \mapsto \mathbb{R}$ such that $x^{i}$ is a selection from its subdifferential mapping. That is,

$$
x^{i}\left(r^{i}\right) \in \partial \varphi\left(r^{i}\right) \quad \forall r^{i} \in T^{i} .
$$

This implies (see Krishna and Maenner (2001), Theorem 1) that for any smooth path $S$ in $T^{i}$ joining $\underline{r}^{i}$ and $\bar{r}^{i}$ the following holds:

$$
\int_{\underline{r}^{i}, S}^{\bar{r}^{i}} x^{i}=\varphi\left(\bar{r}^{i}\right)-\varphi\left(\underline{r}^{i}\right)
$$

So $x^{i}$ is path independent.

$(2) \Rightarrow(1)$ : Let us assume that decomposition monotonicity is satisfied, $f$ satisfies monotonicity and for every agent $i, x^{i}$ is path independent. Take any edge from $T_{f}^{i}$ and denote its starting node $\underline{r}^{i}$ and its ending node $\bar{r}^{i}$. Let $L$ denote the line segment between $\underline{r}^{i}$ and $\bar{r}^{i}$. That is, $L=$ $\left\{r^{i} \in T^{i} \mid r^{i}=(1-\alpha) \underline{r}^{i}+\alpha \bar{r}^{i}, \alpha \in[0,1]\right\}$. Now we pick any $r^{i} \in L$ and substitute the original edge with the path $A=\left(\underline{r}^{i}, r^{i}, \bar{r}^{i}\right)$ which has length $l^{i}\left(\underline{r}^{i}, r^{i}\right)+l^{i}\left(r^{i}, \bar{r}^{i}\right)$. Since decomposition monotonicity is satisfied we have that

$$
l^{i}\left(\underline{r}^{i}, \bar{r}^{i}\right) \geq l^{i}\left(\underline{r}^{i}, r^{i}\right)+l^{i}\left(r^{i}, \bar{r}^{i}\right) .
$$

\footnotetext{
${ }^{3}$ The notion of cyclical monotonicity was introduced by Rockafellar (1966).
} 
That is, the original edge is at least as long as the path $A$. By repeated substitution we can generate a new path $\tilde{A}=\left(r_{1}^{i}=\underline{r}^{i}, \ldots, r_{m}^{i}, r_{m+1}^{i}=\bar{r}^{i}\right)$ where $r_{j}^{i} \in L, \forall j \in\{1, \ldots, m+1\}$. Then (3.9) implies that the original edge is at least as long as $\tilde{A}$. That is,

$$
l^{i}\left(\underline{r}^{i}, \bar{r}^{i}\right) \geq \sum_{j=1}^{m} l^{i}\left(r_{j}^{i}, r_{j+1}^{i}\right),
$$

(see also the example given in Figure 3.4). Since $f$ satisfies monotonicity we have that

$$
l^{i}\left(r_{j}^{i}, r_{j+1}^{i}\right) \geq-l^{i}\left(r_{j+1}^{i}, r_{j}^{i}\right)
$$

Together the above imply that

$$
l^{i}\left(\underline{r}^{i}, \bar{r}^{i}\right) \geq \sum_{j=1}^{m}-l^{i}\left(r_{j+1}^{i}, r_{j}^{i}\right) .
$$

Note that

$$
\begin{aligned}
\sum_{j=1}^{m}-l^{i}\left(r_{j+1}^{i}, r_{j}^{i}\right)= & \sum_{j=1}^{m} E_{-i}\left[v^{i}\left(f\left(r_{j}^{i}, t^{-i}\right) \mid r_{j+1}^{i}, t^{-i}\right)-v^{i}\left(f\left(r_{j+1}^{i}, t^{-i}\right) \mid r_{j+1}^{i}, t^{-i}\right)\right] \\
= & E_{-i}\left[v^{i}\left(f\left(r_{1}^{i}, t^{-i}\right) \mid r_{2}^{i}, t^{-i}\right)-v^{i}\left(f\left(r_{m+1}^{i}, t^{-i}\right) \mid r_{m+1}^{i}, t^{-i}\right)\right] \\
& +\sum_{j=2}^{m} E_{-i}\left[v^{i}\left(f\left(r_{j}^{i}, t^{-i}\right) \mid r_{j+1}^{i}, t^{-i}\right)-v^{i}\left(f\left(r_{j}^{i}, t^{-i}\right) \mid r_{j}^{i}, t^{-i}\right)\right] \\
= & E_{-i}\left[v^{i}\left(f\left(r_{1}^{i}, t^{-i}\right) \mid r_{1}^{i}, t^{-i}\right)-v^{i}\left(f\left(r_{m+1}^{i}, t^{-i}\right) \mid r_{m+1}^{i}, t^{-i}\right)\right] \\
& +\sum_{j=1}^{m} E_{-i}\left[v^{i}\left(f\left(r_{j}^{i}, t^{-i}\right) \mid r_{j+1}^{i}, t^{-i}\right)-v^{i}\left(f\left(r_{j}^{i}, t^{-i}\right) \mid r_{j}^{i}, t^{-i}\right)\right] \\
= & E_{-i}\left[v^{i}\left(f\left(\underline{r}^{i}, t^{-i}\right) \mid \underline{r}^{i}, t^{-i}\right)-v^{i}\left(f\left(\bar{r}^{i}, t^{-i}\right) \mid \bar{r}^{i}, t^{-i}\right)\right] \\
& +\sum_{j=1}^{m} E_{-i}\left[v^{i}\left(f\left(r_{j}^{i}, t^{-i}\right) \mid r_{j+1}^{i}, t^{-i}\right)-v^{i}\left(f\left(r_{j}^{i}, t^{-i}\right) \mid r_{j}^{i}, t^{-i}\right)\right] .
\end{aligned}
$$

The first equality follows from the definition of the edge length given in (3.8). The second equality follows from rearranging the terms of the summation. The third equality is derived by adding and subtracting $E_{-i}\left[v^{i}\left(f\left(r_{1}^{i}, t^{-i}\right) \mid r_{1}^{i}, t^{-i}\right)\right]$. To derive the last equality we use that $r_{1}^{i}=\underline{r}^{i}$ and $r_{m+1}^{i}=\bar{r}^{i}$. By definition of the subgradient we have for any $r_{j}^{i}, r_{j+1}^{i}$ in $\tilde{A}$ that

$$
E_{-i}\left[v^{i}\left(f\left(r_{j}^{i}, t^{-i}\right) \mid r_{j+1}^{i}, t^{-i}\right)-v^{i}\left(f\left(r_{j}^{i}, t^{-i}\right) \mid r_{j}^{i}, t^{-i}\right)\right] \geq\left(r_{j+1}^{i}-r_{j}^{i}\right) x^{i}\left(r_{j}^{i}\right)
$$


Thus, together with the above we get

$$
l^{i}\left(\underline{r}^{i}, \bar{r}^{i}\right) \geq E_{-i}\left[v^{i}\left(f\left(\underline{r}^{i}, t^{-i}\right) \mid \underline{r}^{i}, t^{-i}\right)-v^{i}\left(f\left(\bar{r}^{i}, t^{-i}\right) \mid \bar{r}^{i}, t^{-i}\right)\right]+\sum_{j=1}^{m}\left(r_{j+1}^{i}-r_{j}^{i}\right) x^{i}\left(r_{j}^{i}\right) .
$$

By repeated substitution we can generate paths with more and more edges. In the limit the distance between neighboring nodes goes to zero and

$$
\sum_{j=1}^{m}\left(r_{j+1}^{i}-r_{j}^{i}\right) x^{i}\left(r_{j}^{i}\right) \rightarrow \int_{\underline{r}^{i}, L}^{\bar{r}^{i}} x^{i}
$$

Thus, as $m \rightarrow \infty$,

$$
E_{-i}\left[v^{i}\left(f\left(\underline{r}^{i}, t^{-i}\right) \mid \underline{r}^{i}, t^{-i}\right)-v^{i}\left(f\left(\bar{r}^{i}, t^{-i}\right) \mid \bar{r}^{i}, t^{-i}\right)\right]+\sum_{j=1}^{m}\left(r_{j+1}^{i}-r_{j}^{i}\right) x^{i}\left(r_{j}^{i}\right)
$$

goes to

$$
E_{-i}\left[v^{i}\left(f\left(\underline{r}^{i}, t^{-i}\right) \mid \underline{r}^{i}, t^{-i}\right)-v^{i}\left(f\left(\bar{r}^{i}, t^{-i}\right) \mid \bar{r}^{i}, t^{-i}\right)\right]+\int_{\underline{r}^{i}, L}^{\bar{r}^{i}} x^{i} .
$$

Now, let $C=\left(r_{1}^{i}, \ldots, r_{m}^{i}, r_{m+1}^{i}=r_{1}^{i}\right)$ denote a cycle in $T_{f}^{i}$. Furthermore, let $L_{j}$ denote the line segment between $r_{j}^{i}$ and $r_{j+1}^{i}$. The result in (3.11) and the path independence of $x^{i}$ imply for the length of $C$ that

$$
\begin{aligned}
& \sum_{j=1}^{m} l^{i}\left(r_{j}^{i}, r_{j+1}^{i}\right) \\
\geq & \sum_{j=1}^{m} E_{-i}\left[v^{i}\left(f\left(r_{j}^{i}, t^{-i}\right) \mid r_{j}^{i}, t^{-i}\right)-v^{i}\left(f\left(r_{j+1}^{i}, t^{-i}\right) \mid r_{j+1}^{i}, t^{-i}\right)\right]+\sum_{j=1}^{m} \int_{r_{j}^{i}, L_{j}}^{r_{j+1}^{i}} x^{i} \\
= & 0 .
\end{aligned}
$$

That is, $C$ has non-negative length. In order to see the equality relation, note the following: The terms of the first summation cancel each other out. Furthermore, the second summation describes an integral over a closed path in $T^{i}$ which, due to path independence, equals zero.

Finally, absence of cycles with negative length in $T_{f}^{i}, \forall i \in N$, implies that $f$ is Bayes-Nash incentive compatible (see Theorem 3.1).

If agents' valuation functions satisfy non-decreasing expected differences we can state directly the following: 
Theorem 3.3 Suppose that every agent $i$ has a convex type space and a valuation function satisfying non-decreasing expected differences and yielding expected valuation functions $E_{-i}\left[v^{i}\left(f\left(r^{i}, t^{-i}\right) \mid ., t^{-i}\right)\right]$ which are convex in his true type. Then the following statements are equivalent:

1) $f$ is Bayes-Nash incentive compatible.

2) $f$ satisfies monotonicity and for every agent $i, x^{i}$ is path independent.

\section{Proof}

$(\mathbf{1}) \Rightarrow(\mathbf{2})$ : In order to show the necessity of monotonicity and path independence of $x^{i}$, one can use the corresponding part of the proof for Theorem 3.2 without changes as it does not depend on decomposition monotonicity or non-decreasing expected differences being satisfied.

$(2) \Rightarrow(1)$ : In order to establish the sufficiency of monotonicity and path independence of $x^{i}$, note that monotonicity together with non-decreasing expected differences implies that the costs of manipulation are decomposition monotone, see Lemma 3.1. Finally, we apply Theorem 3.2.

Monotonicity of $f$ and path independence of $x^{i}$ do not imply one another. That monotonicity of $f$ does not imply path independence of $x^{i}$ follows directly from Example 3.1 and Theorem 3.3. It can also be derived directly from Example 3.1. Note that, due to the linearity of the valuation function and there being only one agent in this example, we have $x^{i}(r)=f(r)$. Now, if we consider for example path $A$ consisting of the line segment between the types $x$ and $y$ and path $\tilde{A}$ consisting of the line segment between the types $x$ and $z$ as well as the line segment between the types $z$ and $y$, we find that

$$
\int_{x, A}^{y} f=-\frac{3}{2} \text { and } \int_{x, \tilde{A}}^{y} f=3
$$

So the path integral of $f$ from $x$ to $y$ is not independent of the path of integration. The fact that monotonicity of $f$ does not imply path independence of $x^{i}$ depends crucially upon the assumption of multi-dimensional type spaces in our setting. If we would consider one-dimensional type spaces instead, then monotonicity of $f$ would imply that also $x^{i}$ is monotone, see Lemma 2.2 in the foregoing chapter. This in turn would imply path independence of $x^{i}$.

That path independence does not imply monotonicity is illustrated by the following example.

Example 3.2 Let us consider the allocation of a single, indivisible item. For simplicity we assume that there exists only a single agent to possibly allocate to. He has a type $t \in T=[0,1]$ which reflects the value of the object for him. Given a report $r$ of the agent, the allocation rule $f: T \mapsto[0,1]$ 
assigns to him a probability for getting the object. The agent's valuation for the resulting allocation is $v(f(r) \mid t)=f(r) t$. Thus, in this example we have that $x^{i}(r)=f(r)$. Specifically, we set $f(r)=-(2 r-1)^{2}+1$ (see Figure 3.6). Clearly, $f$ is path independent but not monotone.

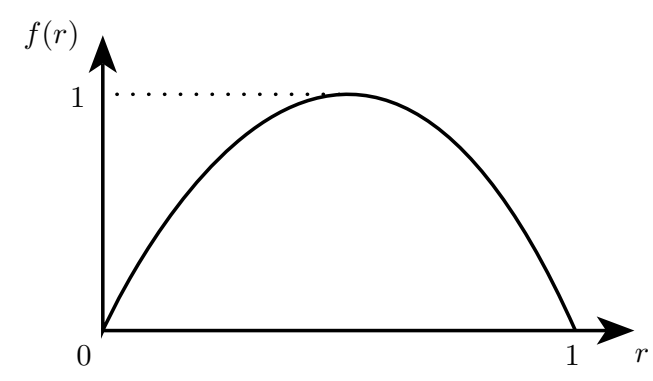

Figure 3.6: The allocation function $f$ in Example 3.2.

\subsubsection{Linear Valuation Functions}

In Müller, Perea and Wolf (2007b) we consider a special case of the setting presented in this chapter. In this special case agents also have multi-dimensional types, convex type spaces and interdependent valuations. However, each agent's valuation function is restricted to be linear in his own true type. Specifically, we have

$$
v^{i}\left(f\left(r^{i}, r^{-i}\right) \mid t^{i}, t^{-i}\right)=\alpha^{i}\left(f\left(r^{i}, r^{-i}\right) \mid t^{-i}\right)+\beta^{i}\left(f\left(r^{i}, r^{-i}\right) \mid t^{-i}\right) t^{i} .
$$

Note that $\alpha^{i}: \Gamma \times T^{-i} \mapsto \mathbb{R}$ and $\beta^{i}: \Gamma \times T^{-i} \mapsto \mathbb{R}^{k}$. That is, $\alpha^{i}$ assigns to every $\left(\gamma, t^{-i}\right) \in \Gamma \times T^{-i}$ a value in $\mathbb{R}$, whereas $\beta^{i}$ assigns to every $\left(\gamma, t^{-i}\right) \in \Gamma \times T^{-i}$ a vector in $\mathbb{R}^{k}$. Thus, agent $i$ 's expected 
valuation is

$$
E_{-i}\left[v^{i}\left(f\left(r^{i}, t^{-i}\right) \mid t^{i}, t^{-i}\right)\right]=E_{-i}\left[\alpha^{i}\left(f\left(r^{i}, t^{-i}\right) \mid t^{-i}\right)\right]+E_{-i}\left[\beta^{i}\left(f\left(r^{i}, t^{-i}\right) \mid t^{-i}\right)\right] t^{i} .
$$

Note that in this linear setting

$$
x^{i}\left(r^{i}\right)=E_{-i}\left[\beta^{i}\left(f\left(r^{i}, t^{-i}\right) \mid t^{-i}\right)\right] .
$$

Hence, $E_{-i}\left[\beta^{i}\left(f\left(., t^{-i}\right) \mid t^{-i}\right)\right]$ is a vector field $T^{i} \mapsto \mathbb{R}^{k}$. Applying (3.12), the monotonicity condition (see Definition 3.2) becomes

$$
E_{-i}\left[\beta^{i}\left(f\left(r^{i}, t^{-i}\right) \mid t^{-i}\right)-\beta^{i}\left(f\left(\tilde{r}^{i}, t^{-i}\right) \mid t^{-i}\right)\right]\left(r^{i}-\tilde{r}^{i}\right) \geq 0 \quad \forall r^{i}, \tilde{r}^{i} \in T^{i}, \forall i \in N
$$

Since our linear valuation functions satisfy the non-decreasing expected differences condition, we readily get the following characterization result for this linear setting.

Theorem 3.4 Suppose that every agent $i$ has a convex type space and a valuation function which is linear in his true type. Then the following statements are equivalent:

1) $f$ is Bayes-Nash incentive compatible.

2) $f$ satisfies weak monotonicity and for every agent $i, E_{-i}\left[\beta^{i}\left(f\left(., t^{-i}\right) \mid t^{-i}\right)\right]$ is path independent.

The above result follows directly from Theorem 3.3. The proof presented in Müller, Perea and Wolf (2007b) works along the same lines as the proof for Theorem 3.2. However, thanks to (3.13) it is somewhat shorter. Due to (3.13) one can work directly with path lengths rather than bound the path lengths using $x^{i}$.

Jehiel and Moldovanu (2001) and Jehiel, Moldovanu and Stacchetti (1999) consider special cases of this linear setting, see Section 3.6 for details. The setting of Jehiel, Moldovanu and Stacchetti (1999) does not feature interdependent valuations. In Jehiel and Moldovanu (2001) the interdependencies with other agents' types enter an agent's valuation function only additively via the $\alpha$-term. The same goes for the one-dimensional setting presented by Myerson (1981), see Section 2.6.1 in the foregoing chapter. There the interdependencies also only come in additively via the $\alpha$-term. However, note that the class of valuation functions considered in Müller, Perea and Wolf (2007b) also allows for interesting settings where the interdependencies with other agents' types enter multiplicatively via the $\beta$-term. Consider for example the following simple, one-dimensional communication network setting: 
Example 3.3 There are three agents, $N=\{1,2,3\}$, each owning a link in the communication network presented in Figure 3.7. That is, agent $i$ owns the link $l_{i}$. Furthermore, there exists a social

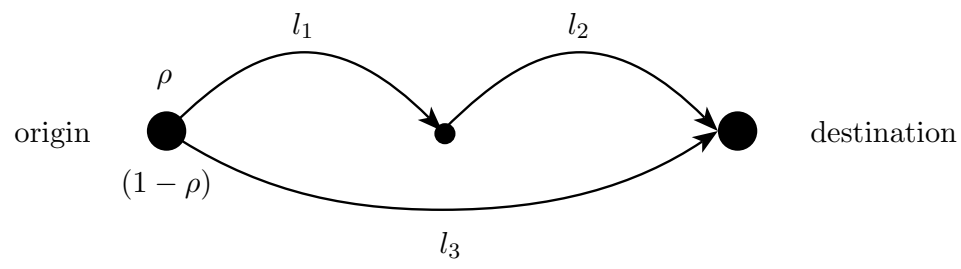

Figure 3.7: The communication network considered in Example 3.3.

planner who wants to send data from the point of origin to the destination point. In order to do so, he can rent different combinations of links. The outcome set $\Gamma$ contains the possible link combinations he can choose from. That is, $\Gamma=\left\{\emptyset,\left\{l_{3}\right\},\left\{l_{1}, l_{2}\right\},\left\{l_{1}, l_{2}, l_{3}\right\}\right\}$. The planner assigns a value $v^{0}$ to the successful data transfer. Data can only be sent once. If the planner chooses the link combination $\left\{l_{1}, l_{2}, l_{3}\right\}$, we assume that the following simple rooting policy is employed: with probability $\rho$ the upper connection $\left\{l_{1}, l_{2}\right\}$ is used, and with probability $1-\rho$ the lower connection $l_{3}$ is used.

Each agent has a type $t^{i} \in[0,1]$ reflecting the probability that his link actually works if the planner tries to send data through it (for example the link might be busy putting through other data). Furthermore, agent $i$ incurs fixed, publicly known costs $c^{i}$ for putting data through his link. His valuation for $\gamma \in \Gamma$ is $v^{i}\left(\gamma \mid t^{i}\right)=-c^{i} b^{i}(\gamma) t^{i}$, where $b^{i}(\gamma)$ denotes the probability that the data reaches the link $l_{i}$ if outcome $\gamma$ is chosen. The allocation rule of the planner is to pick a $\gamma \in \Gamma$ such that $\left[v^{0} H(\gamma)-C(\gamma)\right]$ is maximized. $H(\gamma)$ denotes the expected throughput, that is, the probability that the data reaches the destination given the link combination $\gamma . C(\gamma)$ denotes the expected throughput costs, that is, $\sum_{i} c^{i} b^{i}(\gamma) t^{i}$. The valuations of the agents for the different outcomes are summarized in Table 3.1. Note that agent 1's type enters agent 2's valuation of the outcomes $\left\{l_{1}, l_{2}\right\}$ and $\left\{l_{1}, l_{2}, l_{3}\right\}$

\begin{tabular}{|c||c|c|c|}
\hline \multicolumn{1}{|c||}{ Outcome } & \multicolumn{3}{|c|}{ Agents' valuations } \\
\cline { 2 - 4 }$\gamma$ & $v^{1}\left(\gamma \mid t^{1}\right)$ & $v^{2}\left(\gamma \mid t^{2}\right)$ & $v^{3}\left(\gamma \mid t^{3}\right)$ \\
\hline \hline \multirow{1}{0}{} & 0 & 0 & 0 \\
$\left\{l_{3}\right\}$ & 0 & 0 & $-c^{3} t^{3}$ \\
\cline { 2 - 4 }$\left\{l_{1}, l_{2}\right\}$ & $-c^{1} t^{1}$ & $-c^{2} t^{1} t^{2}$ & 0 \\
\cline { 2 - 4 }$\left\{l_{1}, l_{2}, l_{3}\right\}$ & $-c^{1} \rho t^{1}$ & $-c^{2} \rho t^{1} t^{2}$ & $-c^{3}(1-\rho) t^{3}$ \\
\cline { 2 - 4 } & &
\end{tabular}

Table 3.1: Agents' valuations in Example 3.3.

in the aforementioned multiplicative fashion. Employing different routing policies and data sending 
strategies, one can construct also examples where each agent exhibits such interdependent valuations.

\subsection{Payment Rules}

In the foregoing section we characterized Bayes-Nash incentive compatible allocation rules in terms of a monotonicity condition and an integrability condition. In this section we start out by showing how to construct a corresponding payment rule for an incentive compatible allocation rule. Afterwards we discuss the issue of revenue equivalence. The outline of this section follows closely the one of the corresponding section in the chapter concerned with one-dimensional type settings. Although the general ideas are the same, some minor adjustments have to be made to accommodate for the now multi-dimensional type setting.

According to Definition 3.1 an allocation rule $f$ is Bayes-Nash incentive compatible if there exists a payment rule $P$ such that all the incentive compatibility constraints (3.3) are satisfied. With Theorem 3.1 (see Section 3.3) we establish that the existence of such a payment rule can be guaranteed if for all agents the corresponding networks $T_{f}^{i}$ do not contain any negative length cycles. In the proof of the theorem we construct a payment rule by making use of shortest path lengths. Specifically, for every agent $i$ we pick an arbitrary source node $r_{0}^{i}$ and define for all nodes $r^{i}$

$$
p^{i}\left(r^{i}\right)=\inf \sum_{j=1}^{m} l^{i}\left(r_{j}^{i}, r_{j+1}^{i}\right),
$$

where the infimum is taken over all paths $A=\left(r_{1}^{i}=r^{i}, \ldots, r_{m+1}^{i}=r_{0}^{i}\right)$ in $T_{f}^{i}$, that is, all paths that start at node $r^{i}$ and end at the source node $r_{0}^{i}$. The payment rule $P$ is then constructed by setting

$$
P_{i}\left(r^{i}, t^{-i}\right)=p^{i}\left(r^{i}\right) \quad \forall t^{-i} \in T^{-i}
$$

Thus, the payment agent $i$ has to make for reporting $r^{i}$ corresponds to the length of the shortest path in $T_{f}^{i}$ from the node $r^{i}$ to the source node.

As noted at the end of the proof for Theorem 3.1, it is actually sufficient to choose a payment rule such that

$$
E_{-i}\left[P_{i}\left(r^{i}, t^{-i}\right)\right]=p^{i}\left(r^{i}\right)+c^{i}
$$

That is, the expected payment agent $i$ has to make for reporting $r^{i}$ corresponds to the length of the shortest path from the node $r^{i}$ to the source node plus possibly some constant. Thus, we can observe that there is potentially room for a variety of payment rules that make the allocation rule 
$f$ Bayes-Nash incentive compatible. First of all, even for a fixed source node there can exist several payment rules yielding the same expected payments up to an additive constant. Secondly, changing the source node might lead to payment rules yielding different expected payments. Thirdly, there might exist payment rules that do not evolve from shortest path lengths.

However, in the remainder of this section we establish that for the setting discussed in this chapter (where agents have convex type spaces and expected valuation functions which are convex in their own types) actually all payment rules that make an allocation rule $f$ Bayes-Nash incentive compatible yield the same expected payments for the agents up to an additive constant. Such an allocation rule is said to be satisfying revenue equivalence. Krishna and Maenner (2001) show that if agents' type spaces are convex and their valuation functions are convex in their own types then every BayesNash incentive compatible allocation rule satisfies revenue equivalence. Heydenreich, Müller, Uetz and Vohra (2009) are concerned with dominant strategy incentive compatible allocation rules and the characterization of revenue equivalence based on a graph theoretic argument. In an application section they establish that their approach has a natural extension to other notions of incentive compatibility as well. Specifically, they show that a Bayes-Nash incentive compatible allocation rule satisfies revenue equivalence if and only if for every agent $i$ and all types $s^{i}, t^{i} \in T^{i}$ the length of the shortest path from $s^{i}$ to $t^{i}$ and the length of the shortest path from $t^{i}$ to $s^{i}$ add up to zero. Furthermore, Heydenreich, Müller, Uetz and Vohra (2009) establish that the above mentioned result of Krishna and Maenner (2001) follows as an immediate consequence of their results in case the outcome space is countable.

The multi-dimensional type setting discussed in the chapter (see also Section 3.2) imposes the same conditions as imposed by Krishna and Maenner (2001). We show that in this setting all BayesNash incentive compatible allocation rules satisfy revenue equivalence. Although by doing this we do not extend the result of Krishna and Maenner (2001), we provide an alternate proof using our network based approach.

We begin by formally introducing the definition of revenue equivalence:

Definition 3.6 (Revenue Equivalence) Suppose the allocation rule $f$ is Bayes-Nash incentive compatible. We say that $f$ satisfies revenue equivalence if for any two payment rules $P, \tilde{P}$ such that all the incentive compatibility constraints (3.3) are satisfied there exists a constant $c^{i}$ such that we have

$$
E_{-i}\left[P_{i}\left(t^{i}, t^{-i}\right)\right]=E_{-i}\left[\tilde{P}_{i}\left(t^{i}, t^{-i}\right)\right]+c^{i} \quad \forall t^{i} \in T^{i}, \forall i \in N
$$

So, an allocation rule $f$ satisfies revenue equivalence if all payment rules that make $f$ Bayes-Nash 
incentive compatible yield the same expected payments up to an additive constant. Our notion of revenue equivalence is equivalent to what is termed payoff equivalence in Krishna and Maenner (2001).

Suppose that the allocation rule $f$ is Bayes-Nash incentive compatible. Furthermore, suppose that $P$ is a payment rule such that all the incentive constraints in (3.3) are satisfied. Now let us consider agent $i$ and some types $s^{i}, t^{i} \in T^{i}$. Based on (3.3) and (3.8) we have that

$$
E_{-i}\left[P_{i}\left(s^{i}, t^{-i}\right)-P_{i}\left(t^{i}, t^{-i}\right)\right] \leq l^{i}\left(s^{i}, t^{i}\right)
$$

as well as that

$$
E_{-i}\left[P_{i}\left(t^{i}, t^{-i}\right)-P_{i}\left(s^{i}, t^{-i}\right)\right] \leq l^{i}\left(t^{i}, s^{i}\right)
$$

Together the above inequalities yield

$$
-l^{i}\left(t^{i}, s^{i}\right) \leq E_{-i}\left[P_{i}\left(s^{i}, t^{-i}\right)-P_{i}\left(t^{i}, t^{-i}\right)\right] \leq l^{i}\left(s^{i}, t^{i}\right) .
$$

That is, for agent $i$ the difference in expected payments for reporting $s^{i}$ or $t^{i}$ is bounded by the length of the edge from $s^{i}$ to $t^{i}$ and the negative length of the backward edge.

Now consider a sequence of nodes $A=\left(r_{1}^{i}=s^{i}, \ldots, r_{m}^{i}, r_{m+1}^{i}=t^{i}\right)$ which starts at $s^{i}$ and ends at $t^{i}$. Parallel to the above we have for any $r_{j}^{i}, r_{j+1}^{i}$ in $A$ that

$$
E_{-i}\left[P_{i}\left(r_{j}^{i}, t^{-i}\right)-P_{i}\left(r_{j+1}^{i}, t^{-i}\right)\right] \leq l^{i}\left(r_{j}^{i}, r_{j+1}^{i}\right)
$$

and that

$$
E_{-i}\left[P_{i}\left(r_{j+1}^{i}, t^{-i}\right)-P_{i}\left(r_{j}^{i}, t^{-i}\right)\right] \leq l^{i}\left(r_{j+1}^{i}, r_{j}^{i}\right)
$$

Similar to (3.14) together this yields

$$
-l^{i}\left(r_{j+1}^{i}, r_{j}^{i}\right) \leq E_{-i}\left[P_{i}\left(r_{j}^{i}, t^{-i}\right)-P_{i}\left(r_{j+1}^{i}, t^{-i}\right)\right] \leq l^{i}\left(r_{j}^{i}, r_{j+1}^{i}\right) .
$$

By summing up the inequalities (3.15) for all elements in $A$ we get

$$
\sum_{j=1}^{m}-l^{i}\left(r_{j+1}^{i}, r_{j}^{i}\right) \leq \sum_{j=1}^{m} E_{-i}\left[P_{i}\left(r_{j}^{i}, t^{-i}\right)-P_{i}\left(r_{j+1}^{i}, t^{-i}\right)\right] \leq \sum_{j=1}^{m} l^{i}\left(r_{j}^{i}, r_{j+1}^{i}\right) .
$$


Observe that

$$
\begin{aligned}
\sum_{j=1}^{m} E_{-i}\left[P_{i}\left(r_{j}^{i}, t^{-i}\right)-P_{i}\left(r_{j+1}^{i}, t^{-i}\right)\right] & =E_{-i}\left[P_{i}\left(r_{1}^{i}, t^{-i}\right)-P_{i}\left(r_{m+1}^{i}, t^{-i}\right)\right] \\
& =E_{-i}\left[P_{i}\left(s^{i}, t^{-i}\right)-P_{i}\left(t^{i}, t^{-i}\right)\right]
\end{aligned}
$$

since all the terms of the summation cancel except for the first one and the last one and since we have that $r_{1}^{i}=s^{i}$ and $r_{m+1}^{i}=t^{i}$. Now, using (3.17) we can write (3.16) as

$$
\sum_{j=1}^{m}-l^{i}\left(r_{j+1}^{i}, r_{j}^{i}\right) \leq E_{-i}\left[P_{i}\left(s^{i}, t^{-i}\right)-P_{i}\left(t^{i}, t^{-i}\right)\right] \leq \sum_{j=1}^{m} l^{i}\left(r_{j}^{i}, r_{j+1}^{i}\right)
$$

So for any sequence of nodes starting at $s^{i}$ and ending at $t^{i}$, the difference in agent $i$ 's expected payments for reporting $s^{i}$ or $t^{i}$ is bounded by the length of the path from $s^{i}$ to $t^{i}$ along these nodes and the negative length of the reverse path along the same nodes. For the setting considered in this chapter (see Section 3.2) we can guarantee that there exists a sequence of nodes such that the left-hand side and the right-hand side of (3.18) are equal and hence, the allocation rule $f$ satisfies revenue equivalence.

Theorem 3.5 Suppose that every agent $i$ has a convex type space $T^{i}$ and expected valuation functions $E_{-i}\left[v^{i}\left(f\left(r^{i}, t^{-i}\right) \mid ., t^{-i}\right)\right]$ which are convex in his true type. If the allocation rule $f$ is Bayes-Nash incentive compatible then it also satisfies revenue equivalence.

\section{Proof}

Let us assume that each agent has a convex type space and expected valuation functions which are convex in his true type. Furthermore, let us assume that the allocation rule $f$ is Bayes-Nash incentive compatible. Let $P$ be a payment rule such that all the incentive compatibility constraints in (3.3) are satisfied.

Take agent $i$ and pick two types $s^{i}, t^{i} \in T^{i}$. Let $L$ denote the line segment between $s^{i}$ and $t^{i}$. Now, consider a sequence of nodes between $s^{i}$ and $t^{i}$ along the line segment $L$, $A=\left(r_{1}^{i}=s^{i}, \ldots, r_{m}^{i}, r_{m+1}^{i}=t^{i}\right)$. Recall from (3.18) that

$$
\sum_{j=1}^{m}-l^{i}\left(r_{j+1}^{i}, r_{j}^{i}\right) \leq E_{-i}\left[P_{i}\left(s^{i}, t^{-i}\right)-P_{i}\left(t^{i}, t^{-i}\right)\right] \leq \sum_{j=1}^{m} l^{i}\left(r_{j}^{i}, r_{j+1}^{i}\right) .
$$


Note that

$$
\begin{aligned}
\sum_{j=1}^{m} l^{i}\left(r_{j}^{i}, r_{j+1}^{i}\right)= & \sum_{j=1}^{m} E_{-i}\left[v^{i}\left(f\left(r_{j}^{i}, t^{-i}\right) \mid r_{j}^{i}, t^{-i}\right)-v^{i}\left(f\left(r_{j+1}^{i}, t^{-i}\right) \mid r_{j}^{i}, t^{-i}\right)\right] \\
= & E_{-i}\left[v^{i}\left(f\left(r_{1}^{i}, t^{-i}\right) \mid r_{1}^{i}, t^{-i}\right)-v^{i}\left(f\left(r_{m+1}^{i}, t^{-i}\right) \mid r_{m}^{i}, t^{-i}\right)\right] \\
& +\sum_{j=1}^{m-1} E_{-i}\left[v^{i}\left(f\left(r_{j+1}^{i}, t^{-i}\right) \mid r_{j+1}^{i}, t^{-i}\right)-v^{i}\left(f\left(r_{j+1}^{i}, t^{-i}\right) \mid r_{j}^{i}, t^{-i}\right)\right] \\
= & E_{-i}\left[v^{i}\left(f\left(r_{1}^{i}, t^{-i}\right) \mid r_{1}^{i}, t^{-i}\right)-v^{i}\left(f\left(r_{m+1}^{i}, t^{-i}\right) \mid r_{m+1}^{i}, t^{-i}\right)\right] \\
& +\sum_{j=1}^{m} E_{-i}\left[v^{i}\left(f\left(r_{j+1}^{i}, t^{-i}\right) \mid r_{j+1}^{i}, t^{-i}\right)-v^{i}\left(f\left(r_{j+1}^{i}, t^{-i}\right) \mid r_{j}^{i}, t^{-i}\right)\right] \\
= & E_{-i}\left[v^{i}\left(f\left(s^{i}, t^{-i}\right) \mid s^{i}, t^{-i}\right)-v^{i}\left(f\left(t^{i}, t^{-i}\right) \mid t^{i}, t^{-i}\right)\right] \\
& +\sum_{j=1}^{m} E_{-i}\left[v^{i}\left(f\left(r_{j+1}^{i}, t^{-i}\right) \mid r_{j+1}^{i}, t^{-i}\right)-v^{i}\left(f\left(r_{j+1}^{i}, t^{-i}\right) \mid r_{j}^{i}, t^{-i}\right)\right]
\end{aligned}
$$

The first equality follows from the definition of the edge length given in (3.8). The second equality stems from rearranging the terms of the summation. The third equality is derived by adding and subtracting $E_{-i}\left[v^{i}\left(f\left(r_{m+1}^{i}, t^{-i}\right) \mid r_{m+1}^{i}, t^{-i}\right)\right]$. To establish the last equality we use that $r_{1}^{i}=s^{i}$ and $r_{m+1}^{i}=t^{i}$. In a similarly fashion we get that

$$
\begin{aligned}
\sum_{j=1}^{m}-l^{i}\left(r_{j+1}^{i}, r_{j}^{i}\right)= & E_{-i}\left[v^{i}\left(f\left(s^{i}, t^{-i}\right) \mid s^{i}, t^{-i}\right)-v^{i}\left(f\left(t^{i}, t^{-i}\right) \mid t^{i}, t^{-i}\right)\right] \\
& +\sum_{j=1}^{m} E_{-i}\left[v^{i}\left(f\left(r_{j}^{i}, t^{-i}\right) \mid r_{j+1}^{i}, t^{-i}\right)-v^{i}\left(f\left(r_{j}^{i}, t^{-i}\right) \mid r_{j}^{i}, t^{-i}\right)\right] .
\end{aligned}
$$

For a detailed description of the transformation steps involved see (3.10) in the foregoing section. Using (3.20) and (3.19) we can now write (3.18) as follows

$$
\begin{aligned}
& E_{-i}\left[v^{i}\left(f\left(s^{i}, t^{-i}\right) \mid s^{i}, t^{-i}\right)-v^{i}\left(f\left(t^{i}, t^{-i}\right) \mid t^{i}, t^{-i}\right)\right] \\
& +\sum_{j=1}^{m} E_{-i}\left[v^{i}\left(f\left(r_{j}^{i}, t^{-i}\right) \mid r_{j+1}^{i}, t^{-i}\right)-v^{i}\left(f\left(r_{j}^{i}, t^{-i}\right) \mid r_{j}^{i}, t^{-i}\right)\right] \\
\leq & E_{-i}\left[P_{i}\left(s^{i}, t^{-i}\right)-P_{i}\left(t^{i}, t^{-i}\right)\right] \\
\leq & E_{-i}\left[v^{i}\left(f\left(s^{i}, t^{-i}\right) \mid s^{i}, t^{-i}\right)-v^{i}\left(f\left(t^{i}, t^{-i}\right) \mid t^{i}, t^{-i}\right)\right] \\
& +\sum_{j=1}^{m} E_{-i}\left[v^{i}\left(f\left(r_{j+1}^{i}, t^{-i}\right) \mid r_{j+1}^{i}, t^{-i}\right)-v^{i}\left(f\left(r_{j+1}^{i}, t^{-i}\right) \mid r_{j}^{i}, t^{-i}\right)\right] .
\end{aligned}
$$


By definition of the subgradient we have for any $r_{j}^{i}, r_{j+1}^{i}$ in $A$ that

$$
E_{-i}\left[v^{i}\left(f\left(r_{j}^{i}, t^{-i}\right) \mid r_{j+1}^{i}, t^{-i}\right)-v^{i}\left(f\left(r_{j}^{i}, t^{-i}\right) \mid r_{j}^{i}, t^{-i}\right)\right] \geq\left(r_{j+1}^{i}-r_{j}^{i}\right) x^{i}\left(r_{j}^{i}\right),
$$

(for definition of $x^{i}($.$) see (3.2)). This implies that$

$$
\sum_{j=1}^{m} E_{-i}\left[v^{i}\left(f\left(r_{j}^{i}, t^{-i}\right) \mid r_{j+1}^{i}, t^{-i}\right)-v^{i}\left(f\left(r_{j}^{i}, t^{-i}\right) \mid r_{j}^{i}, t^{-i}\right)\right] \geq \sum_{j=1}^{m}\left(r_{j+1}^{i}-r_{j}^{i}\right) x^{i}\left(r_{j}^{i}\right) .
$$

Similarly we have that

$$
E_{-i}\left[v^{i}\left(f\left(r_{j+1}^{i}, t^{-i}\right) \mid r_{j}^{i}, t^{-i}\right)-v^{i}\left(f\left(r_{j+1}^{i}, t^{-i}\right) \mid r_{j+1}^{i}, t^{-i}\right)\right] \geq\left(r_{j}^{i}-r_{j+1}^{i}\right) x^{i}\left(r_{j+1}^{i}\right)
$$

Multiplying both sides by -1 yields

$$
E_{-i}\left[v^{i}\left(f\left(r_{j+1}^{i}, t^{-i}\right) \mid r_{j+1}^{i}, t^{-i}\right)-v^{i}\left(f\left(r_{j+1}^{i}, t^{-i}\right) \mid r_{j}^{i}, t^{-i}\right)\right] \leq\left(r_{j+1}^{i}-r_{j}^{i}\right) x^{i}\left(r_{j+1}^{i}\right)
$$

which implies that

$$
\sum_{j=1}^{m} E_{-i}\left[v^{i}\left(f\left(r_{j+1}^{i}, t^{-i}\right) \mid r_{j+1}^{i}, t^{-i}\right)-v^{i}\left(f\left(r_{j+1}^{i}, t^{-i}\right) \mid r_{j}^{i}, t^{-i}\right)\right] \leq \sum_{j=1}^{m}\left(r_{j+1}^{i}-r_{j}^{i}\right) x^{i}\left(r_{j+1}^{i}\right) .
$$

Applying (3.22) and (3.23) to (3.21) yields

$$
\begin{aligned}
& E_{-i}\left[v^{i}\left(f\left(s^{i}, t^{-i}\right) \mid s^{i}, t^{-i}\right)-v^{i}\left(f\left(t^{i}, t^{-i}\right) \mid t^{i}, t^{-i}\right)\right]+\sum_{j=1}^{m}\left(r_{j+1}^{i}-r_{j}^{i}\right) x^{i}\left(r_{j}^{i}\right) \\
\leq & E_{-i}\left[P_{i}\left(s^{i}, t^{-i}\right)-P_{i}\left(t^{i}, t^{-i}\right)\right] \\
\leq & E_{-i}\left[v^{i}\left(f\left(s^{i}, t^{-i}\right) \mid s^{i}, t^{-i}\right)-v^{i}\left(f\left(t^{i}, t^{-i}\right) \mid t^{i}, t^{-i}\right)\right]+\sum_{j=1}^{m}\left(r_{j+1}^{i}-r_{j}^{i}\right) x^{i}\left(r_{j+1}^{i}\right) .
\end{aligned}
$$

Observe that $x^{i}$ is path independent. (This is a necessary condition for $f$ being Bayes-Nash incentive compatible. For details the reader is referred to the first part of the proof for Theorem 3.2 
in the foregoing section.) Considering all the nodes along the line segment $L$, it follows that

$$
\begin{aligned}
& E_{-i}\left[v^{i}\left(f\left(s^{i}, t^{-i}\right) \mid s^{i}, t^{-i}\right)-v^{i}\left(f\left(t^{i}, t^{-i}\right) \mid t^{i}, t^{-i}\right)\right]+\int_{s^{i}, L}^{t^{i}} x^{i} \\
\leq & E_{-i}\left[P_{i}\left(s^{i}, t^{-i}\right)-P_{i}\left(t^{i}, t^{-i}\right)\right] \\
\leq & E_{-i}\left[v^{i}\left(f\left(s^{i}, t^{-i}\right) \mid s^{i}, t^{-i}\right)-v^{i}\left(f\left(t^{i}, t^{-i}\right) \mid t^{i}, t^{-i}\right)\right]+\int_{s^{i}, L}^{t^{i}} x^{i},
\end{aligned}
$$

and we get that for all $s^{i}, t^{i} \in T^{i}$

$$
\begin{aligned}
& E_{-i}\left[P_{i}\left(s^{i}, t^{-i}\right)-P_{i}\left(t^{i}, t^{-i}\right)\right] \\
= & E_{-i}\left[v^{i}\left(f\left(s^{i}, t^{-i}\right) \mid s^{i}, t^{-i}\right)-v^{i}\left(f\left(t^{i}, t^{-i}\right) \mid t^{i}, t^{-i}\right)\right]+\int_{s^{i}, L}^{t^{i}} x^{i} .
\end{aligned}
$$

Since $x^{i}$ is path independent, the difference in expected payments $E_{-i}\left[P_{i}\left(s^{i}, t^{-i}\right)-P_{i}\left(t^{i}, t^{-i}\right)\right]$ is the same if we pick some path from $s^{i}$ to $t^{i}$ other than the direct line segment $L$. So, up to an additive constant, the expected payments of an agent are completely defined by his valuation function and the allocation rule $f$. Thus, $f$ satisfies revenue equivalence.

\subsection{Applications}

Jehiel and Moldovanu (2001) consider a social choice setting in which agents have convex types spaces and interdependent valuations. Jehiel, Moldovanu and Stacchetti (1999) deal with a single item auction setting where agents have convex types spaces and independent valuations. The later can be folded into the setting of Jehiel and Moldovanu (2001). In both cases agents' valuation functions are linear in their types.

Jehiel and Moldovanu (2001) and Jehiel, Moldovanu and Stacchetti (1999) show that a mechanism $(f, P)$ is Bayes-Nash incentive compatible if and only if monotonicity and path independence are satisfied, and a condition on agents' utility functions holds. This condition requires that for all agents $i$ and all types $s^{i}, t^{i} \in T^{i}$

$$
U^{i}\left(s^{i} \mid s^{i}\right)=U^{i}\left(t^{i} \mid t^{i}\right)+\int_{t^{i}, S}^{s^{i}} q^{i}
$$

where $U^{i}\left(s^{i} \mid s^{i}\right)$ denotes agent $i$ 's expected utility for truthfully reporting $s^{i}$ (see (3.1) for definition). The vector field $q^{i}$ represents expected winning probabilities and depends on the allocation rule. $S$ 
is some path of integration between $t^{i}$ and $s^{i}$. The above can be written as

$$
\begin{aligned}
& E_{-i}\left[P_{i}\left(t^{i}, t^{-i}\right)-P_{i}\left(s^{i}, t^{-i}\right)\right] \\
= & E_{-i}\left[v^{i}\left(f\left(t^{i}, t^{-i}\right) \mid t^{i}, t^{-i}\right)-v^{i}\left(f\left(s^{i}, t^{-i}\right) \mid s^{i}, t^{-i}\right)\right]+\int_{t^{i}, S}^{s^{i}} q^{i} .
\end{aligned}
$$

Thus, the last condition used for characterizing Bayes-Nash incentive compatibility is revenue equivalence.

In the following we show how the two aforementioned settings can be folded into our framework. Especially, into the linear valuations setting presented in Section 3.4.1. Also, we are going to demonstrate that for the characterization of Bayes-Nash incentive compatible allocation rules one only needs to evoke monotonicity and path independence.

\subsubsection{Interdependent Valuations}

In this application we consider the social choice setting introduced by Jehiel and Moldovanu (2001), a setting which allows for allocative externalities as well as interdependent valuations. In this setting there exists a set of social alternatives $H=\{1, \ldots, h\}$. Given reports from all agents, the allocation rule $f: T \mapsto[0,1]^{h}$ assigns to each social alternative a probability to be chosen. So the outcome set $\Gamma$ is the set of all possible probability profiles. Given a report profile $r \in T$, alternative $k$ 's probability to be chosen is denoted $f_{k}(r)$. The allocation rule must satisfy the probability conditions $\sum_{k=1}^{h} f_{k}(t)=1$ and $0 \leq f_{k}(t) \leq 1, \forall k \in H, \forall t \in T$.

For every agent $i$, the type space $T^{i} \subset \mathbb{R}^{h \times n}$ is assumed to be convex and bounded. Element $t_{k j}^{i}$ of agent $i$ 's type $t^{i} \in T^{i}$ affects agent $j$ 's valuation for social alternative $k \in H$. Given a type profile $t \in T$, agent $i$ 's valuation for some alternative $k \in H$ is $v^{i}(k \mid t)=\sum_{j=1}^{n} a_{k i}^{j} t_{k i}^{j}$. The scalars $a_{k i}^{j}$ are common knowledge and $a_{k i}^{i} \geq 0, \forall i \in N, \forall k \in H$.

Now, take agent $i$ having true type $t^{i}$ and reporting $r^{i}$ while the other agents have true types $t^{-i}$ and reports $r^{-i}$. The value that the agent assigns to the resulting outcome is

$$
v^{i}\left(f\left(r^{i}, r^{-i}\right) \mid t^{i}, t^{-i}\right)=\sum_{k=1}^{h}\left(f_{k}\left(r^{i}, r^{-i}\right) \sum_{j=1}^{n} a_{k i}^{j} t_{k i}^{j}\right) .
$$

Consider agent $i$ reporting $r^{i}$ and assume that the other agents report their types truthfully. Then, 
the agent's expected conditional probability for social alternative $k \in H$ to be chosen is

$$
q_{k}^{i}\left(r^{i}\right)=\int_{T^{-i}} f_{k}\left(r^{i}, t^{-i}\right) \pi^{-i}\left(t^{-i}\right) d t^{-i}
$$

Based on the above, we define the vector field $Q^{i}: T^{i} \mapsto \mathbb{R}^{h \times n}$ as follows. Considering $Q^{i}\left(r^{i}\right)$ we have that, for each $k \in H$, the $k i$ th element is given by $a_{k i}^{i} q_{k}^{i}\left(r^{i}\right)$ and the $k j$ th element is zero for all $j \neq i$. Thus, the agent's expected valuation is

$$
\begin{aligned}
E_{-i}\left[v^{i}\left(f\left(r^{i}, t^{-i}\right) \mid t^{i}, t^{-i}\right)\right] & =\int_{T-i}\left(\sum_{k=1}^{h}\left(f_{k}\left(r^{i}, t^{-i}\right) \sum_{j=1}^{n} a_{k i}^{j} t_{k i}^{j}\right)\right) \pi^{-i}\left(t^{-i}\right) d t^{-i} \\
& =Q^{i}\left(r^{i}\right) t^{i}+\sum_{k=1}^{h} \int_{T-i}\left(f_{k}\left(r^{i}, t^{-i}\right) \sum_{\substack{j \in N \\
j \neq i}} a_{k i}^{j} t_{k i}^{j}\right) \pi^{-i}\left(t^{-i}\right) d t^{-i} .
\end{aligned}
$$

In (3.25) the $Q^{i}\left(r^{i}\right) t^{i}$ represents the value that agent $i$ attaches to a certain expected allocation. Also entering his expected valuation are the interdependencies with the other agents' types which are captured by the second term in (3.25). Applying (3.25), the monotonicity condition (see Definition 3.2) becomes

$$
\left(Q^{i}\left(r^{i}\right)-Q^{i}\left(\tilde{r}^{i}\right)\right)\left(r^{i}-\tilde{r}^{i}\right) \geq 0 \quad \forall r^{i}, \tilde{r}^{i} \in T^{i}, \forall i \in N
$$

Jehiel and Moldovanu (2001) show that a mechanism $(f, P)$ is Bayes-Nash incentive compatible if and only if (3.26) holds, $Q^{i}$ is path independent $\forall i \in N$ and

$$
U^{i}\left(r^{i} \mid r^{i}\right)=U^{i}\left(\tilde{r}^{i} \mid \tilde{r}^{i}\right)+\int_{\tilde{r}^{i}, S}^{r^{i}} Q^{i} \quad \forall r^{i}, \tilde{r}^{i} \in T^{i}, \forall i \in N
$$

where $S$ denotes a path in $T^{i}$ connecting $\tilde{r}^{i}$ and $r^{i}$. Due to path independence, it does not matter which path of integration is chosen. Remember that $U^{i}\left(r^{i} \mid r^{i}\right)$ denotes agent $i$ 's expected utility for truthfully reporting $r^{i}$ (see (3.1) for definition of expected utilities).

Note that the social choice model under consideration here constitutes a special case of the linear valuations setting presented in Section 3.4.1. More specifically, we are dealing with a special case where the interdependencies with other agents' types enter an agent's valuation function only additively via the $\alpha$-term in (3.12). Thus, we can directly apply the results derived earlier in this chapter. From Theorem 3.4 it follows that $f$ is Bayes-Nash incentive compatible if and only if the monotonicity condition in (3.26) holds and $Q^{i}$ is path independent $\forall i \in N$. Compared to Jehiel and 
Moldovanu(2001), this characterization of Bayes-Nash incentive compatibility is not using condition (3.27). In the following we show how (3.27) follows from the other conditions.

Now, suppose that monotonicity of $f$ and path independence of $Q^{i}$ are satisfied. Before we start constructing a payment rule that makes $f$ Bayes-Nash incentive compatible, we observe the following with regard to path lengths. Take $\underline{r}^{i}, \bar{r}^{i} \in T_{f}^{i}$ and let $L$ denote the line segment between them. Consider a path $A=\left\{r_{1}^{i}=\underline{r}^{i}, \ldots, r_{m+1}^{i}=\bar{r}^{i}\right\}$ from $\underline{r}^{i}$ to $\bar{r}^{i}$ that moves along the line between the two. That is, every $r_{j}^{i}$ is on $L$. By applying (3.25) to (3.19) we find for the length of $A$ that

$$
\begin{aligned}
\sum_{j=1}^{m} l^{i}\left(r_{j}^{i}, r_{j+1}^{i}\right)= & E_{-i}\left[v^{i}\left(f\left(\underline{r}^{i}, t^{-i}\right) \mid \underline{r}^{i}, t^{-i}\right)-v^{i}\left(f\left(\bar{r}^{i}, t^{-i}\right) \mid \bar{r}^{i}, t^{-i}\right)\right] \\
& +\sum_{j=1}^{m} Q^{i}\left(r_{j+1}^{i}\right)\left(r_{j+1}^{i}-r_{j}^{i}\right) .
\end{aligned}
$$

Since agents' valuation functions satisfy non-decreasing expected differences, decomposition monotonicity is satisfied (see Lemma 3.1). So we can construct paths that are shorter than $A$ (or as long) by letting them visit the same nodes as $A$ and also additional nodes along $L$ (see also example in Figure 3.4). In the limit, as $m \rightarrow \infty$, the distance between neighboring nodes goes to zero and

$$
\sum_{j=1}^{m} Q^{i}\left(r_{j+1}^{i}\right)\left(r_{j+1}^{i}-r_{j}^{i}\right) \rightarrow \int_{\underline{r}^{i}, L}^{\bar{r}^{i}} Q^{i} .
$$

Thus, (3.28) goes to

$$
E_{-i}\left[v^{i}\left(f\left(\underline{r}^{i}, t^{-i}\right) \mid \underline{r}^{i}, t^{-i}\right)-v^{i}\left(f\left(\bar{r}^{i}, t^{-i}\right) \mid \bar{r}^{i}, t^{-i}\right)\right]+\int_{\underline{r}^{i}, L}^{\bar{r}^{i}} Q^{i} .
$$

Let us now construct a payment rule that makes $f$ Bayes-Nash incentive compatible. We use the approach utilizing shortest path lengths (see proof of Theorem 3.1 and the beginning of Section 3.5). For each agent $i$ we pick $r_{0}^{i}$ as the source node in $T_{f}^{i}$. We set

$$
p^{i}\left(r^{i}\right)=\inf \sum_{j=1}^{m} l^{i}\left(r_{j}^{i}, r_{j+1}^{i}\right),
$$

where the infimum is taken over all paths $A=\left(r_{1}^{i}=r^{i}, \ldots, r_{m+1}^{i}=r_{0}^{i}\right)$ in $T_{f}^{i}$. Let $L_{j}$ denote the line segment between $r_{j}^{i}$ and $r_{j+1}^{i}$, whereas $L$ denotes the line segment between the source and $r^{i}$. Any payment rule $P$ such that $E_{-i}\left[P_{i}\left(r^{i}, t^{-i}\right)\right]=p^{i}\left(r^{i}\right)$ makes $f$ Bayes-Nash incentive compatible. This can be achieved for example by setting $P_{i}\left(r^{i}, t^{-i}\right)=p^{i}\left(r^{i}\right), \forall t^{-i} \in T^{-i}$. 
Based on what we have established before, if we take some path $A$, we can construct paths that are shorter (or as long) by letting them visit the same nodes as $A$ and also additional nodes along the line segments in between. In the limit, as the number of nodes goes to infinity, the length of the paths goes to

$$
\sum_{j=1}^{m}\left(E_{-i}\left[v^{i}\left(f\left(r_{j}^{i}, t^{-i}\right) \mid r_{j}^{i}, t^{-i}\right)-v^{i}\left(f^{i}\left(r_{j+1}^{i}, t^{-i}\right) \mid r_{j+1}^{i}, t^{-i}\right)\right]+\int_{r_{j}^{i}, L j}^{r_{j+1}^{i}} Q^{i}\right) .
$$

Since $Q^{i}$ is path independent we have that ${ }^{4}$

$$
\sum_{j=1}^{m} \int_{r_{j}^{i}, L j}^{r_{j+1}^{i}} Q^{i}=\int_{r^{i}, L}^{r_{0}^{i}} Q^{i}
$$

So, based on the above, we find for our payment rule that

$$
E_{-i}\left[P_{i}\left(r^{i}, t^{-i}\right)\right]=E_{-i}\left[v^{i}\left(f\left(r^{i}, t^{-i}\right) \mid r^{i}, t^{-i}\right)-v^{i}\left(f\left(r_{0}^{i}, t^{-i}\right) \mid r_{0}^{i}, t^{-i}\right)\right]-\int_{r_{0}^{i}, L}^{r^{i}} Q^{i} .
$$

Note that by construction $E_{-i}\left[P_{i}\left(r_{0}^{i}, t^{-i}\right)\right]=0$. Thus, we can subtract $E_{-i}\left[P_{i}\left(r_{0}^{i}, t^{-i}\right)\right]$ on the left-hand side of (3.29), yielding

$$
\begin{aligned}
E_{-i}\left[P_{i}\left(r^{i}, t^{-i}\right)-P_{i}\left(r_{0}^{i}, t^{-i}\right)\right]= & E_{-i}\left[v^{i}\left(f\left(r^{i}, t^{-i}\right) \mid r^{i}, t^{-i}\right)-v^{i}\left(f\left(r_{0}^{i}, t^{-i}\right) \mid r_{0}^{i}, t^{-i}\right)\right] \\
& -\int_{r_{0}^{i}, L}^{r^{i}} Q^{i} .
\end{aligned}
$$

Since agents have convex type spaces and expected valuation functions which are linear in their true types, it follows from Theorem 3.5 that the allocation rule satisfies revenue equivalence. That is, all payment rules making $f$ Bayes-Nash incentive compatible yield the same expected payments up to an additive constant. So (3.30) holds for all of them. Thus, by rearranging the terms in (3.30), we get

$$
U^{i}\left(r^{i} \mid r^{i}\right)=U^{i}\left(r_{0}^{i} \mid r_{0}^{i}\right)+\int_{r_{0}^{i}, L}^{r^{i}} Q^{i}
$$

as a necessary condition for Bayes-Nash incentive compatibility. (3.27) follows readily.

\footnotetext{
${ }^{4}$ For convenience we picked the line segment $L$ as the path of integration. Due to the path independence of $Q^{i}$, it can be replaced with any other path connecting the source and $r^{i}$.
} 


\subsubsection{Single Item Auction With Externalities}

In this section we consider the single item auction presented by Jehiel, Moldovanu and Stacchetti (1999). In this setting a single, indivisible item is auctioned off to one of $n$ potential buyers. Each buyer has a convex type space $T^{i} \subset \mathbb{R}^{n}$. A buyer's type reflects his value for the different possible outcomes. Suppose buyer $i$ has type $t^{i}$. The $i$ th type element $t_{i}^{i} \in[a, b]$ represents his value for the item. Similarly, the $j$ th type element $t_{j}^{i} \in[\tilde{a}, \tilde{b}]$ represents the value of the externalities he incurs if buyer $j \neq i$ wins the item. Commonly, we have that $t_{i}^{i} \geq 0$ and $t_{j}^{i} \leq 0$. That is, buyer $i$ attaches positive value to winning the item and negative value to someone else getting it. Given reports from all buyers, the allocation rule $f: T \mapsto[0,1]^{n}$ assigns to each buyer a probability for winning the item. So the outcome set $\Gamma$ is the set of all possible winning probability profiles. Buyer $i$ 's probability to win, given a report profile $t \in T$, is denoted $f^{i}(t)$. The allocation rule must satisfy the probability condition $\sum_{i=1}^{n} f^{i}(t) \leq 1, \forall t \in T$.

Buyers' valuations are independent. Let buyer $i$ have true type $t^{i}$ and report $r^{i}$ while the others have true types $t^{-i}$ and make reports $r^{-i}$. The value that the buyer assigns to the resulting allocation is

$$
v^{i}\left(f\left(r^{i}, r^{-i}\right) \mid t^{i}, t^{-i}\right)=f\left(r^{i}, r^{-i}\right) t^{i}
$$

Now, let buyer $i$ report $r^{i}$ and assume that the other buyers report truthfully. His expected conditional probability for buyer $j$ to win the item is

$$
q_{j}^{i}\left(r^{i}\right)=\int_{T^{-i}} f^{j}\left(r^{i}, t^{-i}\right) \pi^{-i}\left(t^{-i}\right) d t^{-i}
$$

We define $q^{i}\left(r^{i}\right)=\left(q_{1}^{i}\left(r^{i}\right), \ldots, q_{n}^{i}\left(r^{i}\right)\right)$. The buyer's expected valuation is

$$
E_{-i}\left[v^{i}\left(f\left(r^{i}, t^{-i}\right) \mid t^{i}, t^{-i}\right)\right]=q^{i}\left(r^{i}\right) t^{i}
$$

Applying (3.31), the monotonicity condition (see Definition 3.2) becomes

$$
\left(q^{i}\left(r^{i}\right)-q^{i}\left(\tilde{r}^{i}\right)\right)\left(r^{i}-\tilde{r}^{i}\right) \geq 0 \quad \forall r^{i}, \tilde{r}^{i} \in T^{i}, \forall i \in N
$$

Jehiel, Moldovanu and Stacchetti (1999) show that a mechanism $(f, P)$ is Bayes-Nash incentive 
compatible if and only if (3.32) holds, $q^{i}$ is path independent $\forall i \in N$ and

$$
U^{i}\left(r^{i} \mid r^{i}\right)=U^{i}\left(\tilde{r}^{i} \mid \tilde{r}^{i}\right)+\int_{\tilde{r}^{i}, S}^{r^{i}} q^{i} \quad \forall r^{i}, \tilde{r}^{i} \in T^{i}, \forall i \in N
$$

where $S$ denotes a path in $T^{i}$ connecting $\tilde{r}^{i}$ and $r^{i}$. Due to path independence, it does not matter which path of integration is chosen.

Observe that the single item auction setting presented in this section, can be folded into the social choice model of Jehiel and Moldovanu (2001). Thus, the reader is referred to Section 3.6.1 for its analysis. Here we just briefly summarize the results. From Theorem 3.4 it follows that $f$ is Bayes-Nash incentive compatible if and only if $f$ satisfies monotonicity and $q^{i}$ is path independent $\forall i \in N$. Using shortest path lengths to construct payments and picking $r_{0}^{i}$ as the source node in $T_{f}^{i}$ yields

$$
E_{-i}\left[P_{i}\left(r^{i}, t^{-i}\right)\right]=q^{i}\left(r^{i}\right) r^{i}-q^{i}\left(r_{0}^{i}\right) r_{0}^{i}-\int_{r_{0}^{i}, L}^{r^{i}} q^{i} .
$$

for our payment rule. Due to buyers' convex type spaces and linear valuation functions we can apply Theorem 3.5. Hence, all allocation rules which are Bayes-Nash incentive compatible in this setting satisfy revenue equivalence. Based on (3.34) we get (3.33) as a necessary condition for Bayes-Nash incentive compatibility. 



\section{Combinatorial Scoring Auctions}

In this chapter we concern ourselves with a combinatorial, multi-attribute procurement mechanism called combinatorial scoring auction. In our setting there are several suppliers which have multidimensional private information. A buyer wants to procure several items at once. Subsets of these items are characterized by a price as well as by a number of non-monetary attributes called quality (for example completion time of a project). The suppliers submit offers specifying prices and quality levels for these subsets. These offers are evaluated according to a quasi-linear scoring rule. Based on the resulting scores, suppliers win contracts for the delivery of certain items. Such a contract only specifies the set of items a supplier has to deliver and a score that he has to meet. The decision about the specific price-quality combination is at the discretion of the supplier. He only has to make sure that his chosen combination is yielding the contracted score. In deciding about the delivered price-quality combination, the supplier aims at optimizing his own profit.

We analyze the equilibria in such auctions. Also, we establish a link between combinatorial scoring auctions and combinatorial price-only auctions. It is demonstrated how this link can be exploited to employ preexisting knowledge about the equilibrium behavior in regular, price-only auctions in order to facilitate the strategic analysis of combinatorial scoring auctions. This entails for example results about equilibria existence and revenue equivalence, as presented in the foregoing chapters. Our work is the multi-item extension to the results of Asker and Cantillon (2008) about single item scoring auctions with one-dimensional pseudo-types. Their setting is a special case of our setting which deals with multi-item scoring auctions and multi-dimensional pseudo-types.

\subsection{Introduction}

The design and analysis of combinatorial auctions is a flourishing field in auction theory. It is drawing the interest of researchers from the area of game theory as well as from the area of computer science. (Or as Roger B. Myerson puts it on the back cover of Cramton, Shoham and Steinberg (2006): 
"Combinatorial auctions are the great frontier of auction theory today, ...".) While single item auctions only allocate one item at a time, combinatorial auctions are used to allocate multiple items all at once. By doing this, complementary or substitutable preferences of the bidders for different item sets can be taken into account. The majority of research conducted on combinatorial auctions (but this also holds for single item auctions) considers the price as the unique strategic dimension for bidders. That is, bidders offer prices for different sets of items. Based on these price bids the items are allocated.

However, there are situations in which auction participants are not only concerned with the price of items but also with other non-monetary attributes of the items (called quality). Take for example the construction of a building. The construction process can be subdivided into several tasks. Contractors can make bids on these tasks. The building owner does not only care about the prices at which these tasks are executed by the contractors. He also cares about non-monetary attributes of these tasks, like the completion time, the quality of the used materials, the construction quality or the probability that the contractor goes bankrupt during the job and leaves the task unfinished. On the other side, due to different levels of specialization, contractors might be able to take over several tasks or only some special tasks. Also, the contractor having the lowest cost for executing a specific task might differ depending on the quality level. That is, the low cost contractor for a task at a low quality level might not be the same as the low cost contractor for the same task at a higher quality level. Thus, it can be important to consider other strategic dimensions than just price.

One way to deal with combinatorial, multi-attribute procurement problems, like the one described above, is using combinatorial scoring auctions. In this chapter we analyze the equilibria in such combinatorial scoring auctions. Specifically, we aim at extending the results of Asker and Cantillon (2008). They consider a setting for single item scoring auctions where item quality and bidders' types are multi-dimensional. For each bidder they construct a one-dimensional pseudo-type which is the maximum level of apparent social surplus that this bidder can generate. "Apparent" social surplus because for its construction the scoring rule is used, which can differ from the buyer's true valuation function. Asker and Cantillon (2008) show that knowledge of bidders' pseudo-type distributions is sufficient for describing equilibrium outcomes and the buyer's expected equilibrium utility. This finding allows them to establish a link between single item scoring auctions and standard single item independent private value (IPV) price-only auctions. Specifically, for each single item scoring auction in their setting they identify a corresponding single item IPV price-only auction in which a bidder's type is his pseudo-type. They then show that the strategic analysis of a scoring auction 
reduces to the strategic analysis of the corresponding price-only auction. In this chapter we establish the multi-item extension of this result. That is, we also construct pseudo-types. In our case these pseudo-types are consisting of the maximum levels of apparent social surplus that a bidder can generate for the different item sets. We also show a link between combinatorial scoring auctions and combinatorial price-only auctions. This link is again established by identifying for each combinatorial scoring auction a corresponding price-only auction.

\subsubsection{Related Work}

Che (1993) analyzes single item scoring auctions where the supplier bidding the highest score is selected to deliver the item. He considers a setting where the item quality is one-dimensional and suppliers' private information is also one-dimensional. Suppliers' production costs depend on the quality level as well as on their own private information. Furthermore, he considers scoring rules that are linear in price. In order to construct equilibria in these auctions, he makes use of the maximum level of social welfare that a supplier can produce, also called a supplier's pseudo-type. These pseudo-types are well defined once the scoring rule is given. By employing a relatively simple change in variables, Che (1993) is able to transform the problem of finding equilibria in these scoring auctions into the problem of finding equilibria in standard single item IPV price-only auctions. The later problem is a well-studied area in the auction literature. He also designs an optimal scoring rule, maximizing the buyer's utility.

Branco (1997) extends Che's (1993) analysis of the independent cost setting to the case where suppliers' production costs are correlated. He characterizes an optimal direct revelation mechanism, the implementation of which requires a two-stage auction. David, Azoulay-Schwartz and Kraus (2002a), (2002b), (2003) and (2006) propose and analyze simultaneous and sequential English auctions on suppliers' scores (with and without deadline). Their settings either allow for two-dimensional quality and two-dimensional private information or for general multi-dimensional quality and onedimensional private information. However, in the settings under their consideration, the functional form of the suppliers' utility functions and the scoring rules is more restrictive than in the foregoing papers. They also identify optimal scoring rules for the considered settings, that is, scoring rules which maximize the buyer's expected utility.

Asker and Cantillon (2009), (2008) extend Che's (1993) analysis to a single item scoring auction setting. This setting allows for multi-dimensional quality as well as multi-dimensional private information for the suppliers. Asker and Cantillon (2009) show that, contrary to Che's (1993) one- 
dimensional setting, the optimal buying mechanism cannot be implemented by a scoring auction with a scoring rule which is linear in price if suppliers' information is multi-dimensional. Furthermore, they analyze the performance of scoring auctions in comparison to the optimal mechanism. Asker and Cantillon (2008) prove that in order to describe equilibrium outcomes in single item scoring auctions, it is sufficient to make use of suppliers' one-dimensional pseudo-types. However, if suppliers' original private information is multi-dimensional, this proof is more involved than using the simple variable transformation employed by Che (1993). As Che (1993), Asker and Cantillon (2008) show that the problem of finding equilibria in single item scoring auctions can be transformed into the problem of finding equilibria in standard single item IPV price-only auctions. In this chapter we deliver the multi-item extension of this result. Furthermore, Asker and Cantillon (2008) find that scoring auctions dominate other procedures for buying differentiated objects, like menu auctions, beauty contests and price-only auctions with minimum quality thresholds.

Milgrom (2000) shows that an item can be efficiently allocated by a scoring auction which employs the buyer's valuation function as the scoring rule and runs a Vickrey auction on suppliers' submitted scores. Suyama and Yokoo (2005) investigate a combinatorial multi-attribute procurement auction setting that allows for multi-dimensional quality as well as for multi-dimensional private information of the suppliers. Their setting is a bit more restrictive than the one considered in this chapter. Specifically, in their setting the vector of quality levels for an item set is composed of the quality vectors of the individual items in the set. They propose a direct revelation Vickrey-Clarke-Groves (VCG) mechanism (Vickrey (1961), Clarke (1971), Groves (1973)) that determines the social surplus maximizing allocation of items and quality levels for the items. In this mechanism it is a dominant strategy for a supplier to report his private information truthfully. The combinatorial VCG scoring auction presented in Section 4.5.1 is quite similar in that it is essentially a direct revelation VCG mechanism based on suppliers' pseudo-types. In our auction the welfare maximizing allocation is determined by the auction mechanism. The optimal quality levels are determined by the suppliers themselves. This combinatorial VCG scoring auction is the multi-item extension of Milgrom's (2000) scoring auction.

In addition to the theoretical work presented above, also some experimental work on single item multi-attribute auctions has been done, see for example: Bichler (2000), Chen-Ritzo, Harrison, Kwasnica and Thomas (2005), Strecker and Seifert (2004) as well as Bichler and Kalagnanam (2005). In these papers theoretical equilibrium predictions are verified and different scoring auction formats are compared. A finding of these experiments is that multi-attribute auction mechanisms dominate 
price-only auction mechanisms in terms of the buyer's and suppliers' utilities.

\subsubsection{Chapter Outline \& Our Contribution}

In Section 4.2 we state some basic assumptions and definitions. Also, we describe our combinatorial scoring auction model which is an extension of the single item scoring auction models considered by Che (1993) and Asker and Cantillon (2008).

Contrary to the commonly analyzed single item scoring auctions, in our setting a buyer wants to procure several items at once. Item sets are characterized by a price as well as by a number of nonmonetary attributes. A supplier's production costs for the different item sets are influenced by the chosen quality level and his type which is his private information. We allow for multi-dimensional, independently distributed private information for the suppliers and multi-dimensional non-monetary attributes for the sets of items. The suppliers submit offers on the different item sets. They specify prices as well as quality levels for the non-monetary attributes. Each offer is evaluated according to a scoring rule which assigns to each price-quality offer for an item set a one-dimensional score. ${ }^{1}$ We only consider scoring rules that are linear in price.

Based on the resulting scores an allocation rule determines which suppliers are contracted to deliver certain sets of items. This yields for each supplier an allocation vector. This allocation vector has an element for each item set (including the empty one) specifying the supplier's probability to win the corresponding item set. (For example, consider the case where the allocation rule is deterministic. Then, one element of a suppliers allocation vector is equal to one, whereas the remaining elements are equal to zero.) The delivery contract for an item set specifies only a score that the winning supplier has to meet. The final decision about the specific price-quality combination for the delivered item set is at the discretion of the winning supplier. The only restriction is that his chosen combination has to yield the contracted score. We find that winning suppliers choose the delivered quality level independent of the score that they contractually have to meet. That is, the contracted score effectively determines the price a winning supplier is charging the buyer for his item set. This finding is analogous to findings in single item scoring auction settings, see for example Che (1993) or Asker and Cantillon (2008).

In Section 4.2.2 we observe the following. Given the scoring rule, the maximum level of apparent social surplus that a supplier with a certain type can generate by producing and subsequently selling a set of items is well defined. We use this finding to construct for each supplier a multi-dimensional

\footnotetext{
${ }^{1}$ For an introduction to scoring rules and multi-attribute decision making in general see Yoon and Hwang (1995).
} 
pseudo-type which has a component for each set of items. Each of these components is defined as the maximum level of apparent social surplus that the supplier can create for the corresponding item set. This definition of pseudo-types is analogous to the definition of one-dimensional pseudo-types in single item scoring auction settings (see Asker and Cantillon (2008)).

Based on the possible pseudo-types, we can partition a supplier's type space into equivalence classes of types yielding the same pseudo-type. A supplier's bidding function assigns a bid to each of his possible types. On a particular equivalence class, a supplier's bidding function is doing one of the following two things. On the one hand, the bidding function can assign the same bid to all types, in which case we say that it is not mixing on this equivalence class. Or the bidding function can assign differing bids to the types, in which case we say that it is mixing on this equivalence class. This mixing can happen in two ways. Given the bidding strategies of the others, if differing bids yield also differing expected allocation vectors for the supplier then we speak of allocation mixing. If differing bids yield the same expected allocation vector then we speak of allocation equivalent mixing.

In Section 4.3 we assume that in equilibrium suppliers' bidding functions employ allocation equivalent mixing only for a zero measure of types. Under this assumption we show (Theorem 4.2) that for the equilibrium analysis of a scoring auction it is sufficient to concentrate on bidding functions which are constant on equivalence classes of types. That is, in equilibrium, except for allocation equivalent mixing for a zero measure of types, suppliers assign the same bid to all types yielding the same pseudo-type. Specifically, we show that for any equilibrium in a scoring auction we can construct a new equilibrium with bidding strategies that are constant on equivalence classes of types. A supplier's new bidding strategy is constructed as follows. We pick a representative type from each equivalence class in his type space and assign the old equilibrium bids for these representative types also to all other types in their respective equivalence classes.

In order to ensure that these new bidding strategies indeed constitute an equilibrium, it is sufficient to show that they differ from the suppliers' original equilibrium bidding strategies only on a set of types with zero measure (see proof of Theorem 4.2). Unproblematic in this respect are the equivalence classes of types on which a supplier's original equilibrium bidding function is not mixing. This is because for these types his new bidding function is identical to his original one. Potentially troublesome are the equivalence classes on which the original bidding function is mixing. The reason is that for those types the new bidding strategy is different from the original one. However, by assumption we have that the set of types for which the original bidding function employs allocation equivalent mixing has measure zero. In addition we prove (Lemma 4.4) that the set of types for 
which the original bidding function employs allocation mixing has also measure zero. ${ }^{2}$ This implies that the set of types for which the original bidding function employs mixing (that is, the union of the two aforementioned sets) has measure zero. Hence, the new bidding strategy is almost everywhere the same as the original one.

Similar to Asker and Cantillon (2008) we utilize two facts in order to establish Lemma 4.4 (stating that the set of types for which suppliers' equilibrium strategies employ allocation mixing has zero measure). First, we construct for every equilibrium in the scoring auction an auxiliary equilibrium (Lemma 4.1) in which suppliers' bidding strategies are based on their pseudo-types. This auxiliary equilibrium is constructed in such a way that a supplier's auxiliary equilibrium bidding strategy specifies a mixed bid for each of his pseudo-types. For a certain pseudo-type, the support of such a mixed bid consists of all the pure bids that the original equilibrium bidding function specifies for the types in the corresponding equivalence class. Second, we prove that for almost all pseudo-types this support does not contain pure bids which yield different expected allocations for the supplier (Lemma 4.3). The first finding, the construction of the auxiliary equilibrium, works out in the same way as in Asker and Cantillon (2008). However, for the second finding we use a different method than Asker and Cantillon (2008). Their techniques do not extend well to settings covering multi-dimensional pseudo-types. As part of our method we encounter again a monotonicity condition. This condition is comparable to the monotonicity conditions presented in Chapters 2 and 3.

In Section 4.5 we show how the existing literature on combinatorial IPV price-only auctions can be used for the strategic analysis of scoring auctions. For example, we show how to establish equilibrium existence and revenue equivalence, both topics that we dealt with in the foregoing two chapters. More specifically, for each scoring auction we identify a corresponding combinatorial price-only auction in which a bidder's type, on which he bases his bidding strategy, is his pseudo-type. With Corollary 4.1 we extend Asker and Cantillon's (2008) "Expected Utility Equivalence"-Theorem for the buyer from single item to combinatorial scoring auctions.

As an illustrative example, we describe in Section 4.5.1 a combinatorial scoring auction that allocates items efficiently. As mentioned earlier in this section, this auction relates to the direct revelation VCG mechanism described by Suyama and Yokoo (2005). It is the multi-item extension of a single item scoring auction analyzed by Milgrom (2000). The above mentioned link to price-only auctions is used to establish an equilibrium in this auction.

We end this chapter with a conclusion in Section 4.6 in which we discuss again some of the

\footnotetext{
${ }^{2}$ We have to exclude allocation equivalent mixing for a non-zero measure of types by assumption because we cannot exclude it analytically like in the case of allocation mixing.
} 
simplifying assumptions that we take.

\subsection{Model \& Definitions}

We consider a setting in which a buyer wants to procure a set of distinct items $A=\{1, \ldots, a\}$. There is a set $N=\{1, \ldots, n\}$ of potential suppliers for these items. Each of these suppliers is able to produce every item. ${ }^{3}$ The buyer does not need to purchase from only one supplier. He can buy different subsets of items from different suppliers. Including the empty set which we associate with index 0 , there are $2^{a}$ possible subsets of items. Each non-empty subset of items $j, j \in\left\{1, \ldots, 2^{a}-1\right\}$, is characterized by a sales price $p_{j} \in \mathbb{R}_{+}$and a quality level $q_{j} \in \mathbb{R}_{+}^{m_{j}}$ for its $m_{j} \geq 1$ non-monetary attributes. In order to ease notation later on let us define $d=2^{a}-1$ and $m=\sum_{j=1}^{d} m_{j}$.

The buyer's valuation for the purchase of item set $j$ with quality level $q_{j}$ at price $p_{j}$ is $v_{j}\left(q_{j}\right)-p_{j}$. Each supplier $i$ has some private information in the form of a type $\theta^{i} \in \Theta^{i}$ with $\Theta^{i} \subset \mathbb{R}^{k_{i}}, k_{i} \geq$ 1. Suppliers' type spaces are assumed to be convex and compact. The set of all complete type profiles $\theta=\left(\theta^{1}, \ldots, \theta^{n}\right)$ is denoted $\Theta$, whereas the set of all type profiles excluding supplier $i$, $\theta^{-i}=\left(\theta^{1}, \ldots, \theta^{i-1}, \theta^{i+1}, \ldots, \theta^{n}\right)$, is denoted $\Theta^{-i}$. Suppliers' types are independently distributed. Assuming that supplier $i$ 's types are distributed according to some density $\gamma^{i}$ on $\Theta^{i}$, the joint density $\gamma^{-i}$ on $\Theta^{-i}$ is then given by

$$
\gamma^{-i}\left(\theta^{-i}\right)=\prod_{\substack{h \in N \\ h \neq i}} \gamma^{h}\left(\theta^{h}\right) .
$$

Supplier $i$ 's type influences his production cost. The cost he incurs for producing the set of items $j$ with quality $q_{j}$ is denoted by $c_{j}^{i}\left(q_{j}, \theta^{i}\right)$. We assume that $c_{j}^{i}$ is continuous as well as strictly increasing in the supplier's type. All cost functions $c_{j}^{i}$ are publicly known. Supplier $i$ 's profit $g_{j}^{i}$ from producing the set of items $j$ with quality $q_{j}$ and selling it at price $p_{j}$ is

$$
g_{j}^{i}\left(p_{j}, q_{j}, \theta^{i}\right)=p_{j}-c_{j}^{i}\left(q_{j}, \theta^{i}\right)
$$

\subsubsection{The Scoring Auction}

The scoring auction works as follows. Based on his type each supplier $i$ makes a price-quality bid $\left(p^{i}, q^{i}\right) \in \mathbb{R}_{+}^{d+m}$ consisting of a price-quality offer for each of the possible non-empty item sets. That

\footnotetext{
${ }^{3}$ In case a supplier is not able to produce certain items, we can model this by letting him bid infinitely high prices for the item sets which he is unable to produce. In this way he is not chosen as the supplier for item sets that he cannot deliver.
} 
is $\left(p^{i}, q^{i}\right)=\left(\left(p_{1}^{i}, q_{1}^{i}\right), \ldots,\left(p_{d}^{i}, q_{d}^{i}\right)\right)$ with $\left(p_{j}^{i}, q_{j}^{i}\right) \in \mathbb{R}_{+}^{1+m_{j}} \cdot{ }^{4}$ There is a scoring rule

$$
S_{j}: \mathbb{R}_{+}^{1+m_{j}} \mapsto \mathbb{R}
$$

for every possible set of items $j$. Using this scoring rule, each price-quality offer $\left(p_{j}, q_{j}\right)$ for the item set $j$ is associated with a one-dimensional score $S_{j}\left(p_{j}, q_{j}\right)$. The scoring rules for all item sets are fixed before the bidding starts and known to every supplier. We assume that the scoring rules are quasi-linear, that is,

$$
S_{j}\left(p_{j}, q_{j}\right)=\phi_{j}\left(q_{j}\right)-p_{j} .
$$

In addition it is assumed that $\phi_{j}$ is continuous and that $\phi_{j}\left(q_{j}\right)-c_{j}^{i}\left(q_{j}, \theta^{i}\right)$ is strictly concave in $q_{j}$ for all $\theta^{i}$.

By applying the scoring rules each supplier's original price-quality bid $\left(p^{i}, q^{i}\right) \in \mathbb{R}_{+}^{d+m}$ is transformed into a vector of scores $s^{i} \in \mathbb{R}^{d}$ consisting of a score $s_{j}^{i} \in \mathbb{R}$ for every set of items $j$. An allocation rule maps each profile of scores $s=\left(s^{1}, \ldots, s^{n}\right)$ into an allocation of items to suppliers (or a distribution over such allocations in case the allocation rule is probabilistic). That is, the allocation rule determines which supplier is allowed to provide which items to the buyer. Every item is provided by at most one supplier. This allows for the case that some items are not procured at all. The allocation rule implies for each supplier $i$ and each profile of scores $s$ an allocation vector $x^{i}(s)=\left(x_{0}^{i}(s), x_{1}^{i}(s) \ldots, x_{d}^{i}(s)\right)$ where $x_{0}^{i}(s)$ denotes the probability that $i$ is awarded the empty set, that is, he does not sell any items to the buyer. Similarly, for $j \in\{1, \ldots, d\}, x_{j}^{i}(s)$ denotes supplier $i$ 's probability for winning the contract giving him the right to provide the buyer with the set of items $j$. In order to simplify notation we define the allocation rule

$$
x: \mathbb{R}^{n d} \mapsto[0,1]^{n 2^{a}}
$$

directly as a mapping that maps each profile of scores $s \in \mathbb{R}^{n d}$ into an allocation vector $x^{i}(s) \in[0,1]^{2^{a}}$ for every supplier $i$.

Based on a profile of scores $s$, the winning score rule

$$
w: \mathbb{R}^{n d} \mapsto \mathbb{R}^{n 2^{a}}
$$

\footnotetext{
${ }^{4}$ We are aware of the potential complexity of suppliers' bids. Specifying a price-quality offer for each of the $2^{a}-1$ non-empty subsets of items is highly impractical for larger sets of items. Bid complexity and representation are generally an issue in combinatorial auctions. However, concerns about the representation of bids and bidding languages go beyond the scope of this chapter and are not addressed henceforth. A comprehensive introduction to bidding languages for combinatorial auctions is given by Nisan (2006).
} 
assigns each supplier $i$ with a vector of winning scores $w^{i}(s)=\left(w_{0}^{i}(s), w_{1}^{i}(s), \ldots, w_{d}^{i}(s)\right)$ where $w_{0}^{i}(s)$ is the payment that $i$ has to make in case he is awarded the empty set. Thus, we allow for the case that a supplier has to make a payment to the buyer if he did not win any delivery contract. For $j \in\{1, \ldots, d\}, w_{j}^{i}(s)$ is the score that supplier $i$ has to meet in case he wins the contract to provide the buyer with item set $j$. That is, he has to provide the set of items with a quality level $q_{j}$ at a price $p_{j}$ such that $S_{j}\left(p_{j}, q_{j}\right)=w_{j}^{i}(s)$. This means that the buyer and the supplier only contract a score for the item set. The actual sales price and the delivered quality is chosen by the supplier in such a way that the contracted score is met.

\subsubsection{Pseudo-types}

Suppose that supplier $i$ of type $\theta^{i}$ has won the contract to provide the set of items $j$ to the buyer and has to meet a score $\omega$. He will choose a price-quality pair $\left(p_{j}, q_{j}\right)$ that maximizes his profit $g_{j}^{i}\left(p_{j}, q_{j}, \theta^{i}\right)$ while meeting the score, that is, $S_{j}\left(p_{j}, q_{j}\right)=\omega$. Using (4.1) and (4.2), supplier $i$ 's optimization problem becomes

$$
\begin{array}{ll} 
& \max _{\left(p_{j}, q_{j}\right)}\left(p_{j}-c_{j}^{i}\left(q_{j}, \theta^{i}\right)\right) \\
\text { s.t. } & \phi_{j}\left(q_{j}\right)-p_{j}=\omega .
\end{array}
$$

Substituting for $p_{j}$ in the objective function yields

$$
\max _{q_{j}}\left(\phi_{j}\left(q_{j}\right)-c_{j}^{i}\left(q_{j}, \theta^{i}\right)\right)-\omega
$$

As can be seen in (4.4), the supplier is choosing the apparent optimal quality level independent of the winning score that he has to meet. We assume that for every $\theta^{i} \in \Theta^{i}$ there exits a $q_{j}^{*}>$ 0 that maximizes $\phi_{j}\left(q_{j}\right)-c_{j}^{i}\left(q_{j}, \theta^{i}\right)$. Together with the strict concavity of $\phi_{j}\left(q_{j}\right)-c_{j}^{i}\left(q_{j}, \theta^{i}\right)$ in $q_{j}$ for all $\theta^{i}$ (see assumptions made earlier) this implies that $q_{j}^{*}$ is the only maximum and hence $\arg \max _{q_{j}}\left(\phi_{j}\left(q_{j}\right)-c_{j}^{i}\left(q_{j}, \theta^{i}\right)\right)$ is well defined. Furthermore, given the continuity of $\phi_{j}\left(q_{j}\right)-$ $c_{j}^{i}\left(q_{j}, \theta^{i}\right)$ (again, see assumptions made earlier), it follows from Berge's (1963, p.116) Theorem of the Maximum that

$$
\max _{q_{j}}\left(\phi_{j}\left(q_{j}\right)-c_{j}^{i}\left(q_{j}, \theta^{i}\right)\right)=\phi_{j}\left(q_{j}^{*}\left(\theta^{i}\right)\right)-c_{j}^{i}\left(q_{j}^{*}\left(\theta^{i}\right), \theta^{i}\right)
$$

is a continuous mapping from $\Theta^{i}$ to $\mathbb{R}$. Since $\Theta^{i}$ is compact and connected, its image under this mapping is also compact and connected. Hence, the set $\left\{\max _{q_{j}}\left(\phi_{j}\left(q_{j}\right)-c_{j}^{i}\left(q_{j}, \theta^{i}\right)\right) \mid \theta^{i} \in \Theta^{i}\right\}$ is a 
closed interval in $\mathbb{R}$.

Based on the above we define for every $\theta^{i}$ in supplier $i$ 's type space

$$
t^{i}\left(\theta^{i}\right)=\left(\max _{q_{1}}\left(\phi_{1}\left(q_{1}\right)-c_{1}^{i}\left(q_{1}, \theta^{i}\right)\right), \ldots, \max _{q_{d}}\left(\phi_{d}\left(q_{d}\right)-c_{d}^{i}\left(q_{d}, \theta^{i}\right)\right)\right)
$$

We call $t^{i}\left(\theta^{i}\right)$ the supplier's pseudo-type. Note that suppliers' pseudo-types are dependent on their types as well as on the chosen scoring rules and the production cost functions. Supplier $i$ 's pseudotype is monotonically decreasing in his type: Take $\hat{\theta}^{i}, \theta^{i} \in \Theta^{i}$ such that $\hat{\theta}^{i}$ is componentwise smaller than $\theta^{i}$. Furthermore, let $q_{j}^{* i}($.) denote the optimal quality level that supplier $i$ picks for item set $j$ in (4.4) based on his type. Then, for each element $j$ of his pseudo-type,

$$
\begin{aligned}
t_{j}^{i}\left(\theta^{i}\right) & =\phi_{j}\left(q_{j}^{* i}\left(\theta^{i}\right)\right)-c_{j}^{i}\left(q_{j}^{* i}\left(\theta^{i}\right), \theta^{i}\right) \\
& <\phi_{j}\left(q_{j}^{* i}\left(\theta^{i}\right)\right)-c_{j}^{i}\left(q_{j}^{* i}\left(\theta^{i}\right), \hat{\theta}^{i}\right) \\
& \leq \phi_{j}\left(q_{j}^{* i}\left(\hat{\theta}^{i}\right)\right)-c_{j}^{i}\left(q_{j}^{* i}\left(\hat{\theta}^{i}\right), \hat{\theta}^{i}\right) \\
& =t_{j}^{i}\left(\hat{\theta}^{i}\right) .
\end{aligned}
$$

Above, the first inequality follows from the fact that production costs are increasing in the supplier's type. The second inequality follows from the optimality of $q_{j}^{* i}($.$) .$

Let $\Theta_{t^{i}}^{i}$ denote the set of all types $\theta^{i} \in \Theta^{i}$ yielding the same $t^{i}$ as pseudo-type, that is, $\Theta_{t^{i}}^{i}=$ $\left\{\theta^{i} \in \Theta^{i} \mid t^{i}\left(\theta^{i}\right)=t^{i}\right\}$. A supplier's pseudo-type space is denoted $T^{i}$ with $T^{i} \subset \mathbb{R}^{d}$. Pseudo-types are privately observed and independently distributed. The distributions are common knowledge (since the scoring rules, the production cost functions and suppliers' type distributions are common knowledge). For technical reasons we make two additional assumptions about the pseudo-type mapping. First of all, if a set of pseudo-types has Lebesgue measure zero then the set of types yielding this set of pseudo-types has also Lebesgue measure zero. ${ }^{5}$ Second, we assume that the pseudo-type space $T^{i}$ is rich enough, so that for every $t^{i} \in T^{i}$ there exists a $\hat{t}^{i} \in T^{i}$ that is either component wise bigger or smaller than $t^{i}$. Furthermore, we require that any two $t^{i}, \hat{t}^{i} \in T^{i}$, where $t^{i}$ is componentwise smaller than $\hat{t}^{i}$, can be connected via a finite sequence of pseudo-types that is stepwise increasing. Figure 4.1 illustrates this "zigzagging" from $t^{i}$ to $\hat{t}^{i}$ for a two-dimensional example.

Suppose that suppliers have the type profile $\theta=\left(\theta^{1}, \ldots, \theta^{n}\right)$ and make reports $\left(p^{1}, q^{1}\right), \ldots$, $\left(p^{n}, q^{n}\right)$ implying the profile of scores $s=\left(s^{1}, \ldots, s^{n}\right)$. The utility that supplier $i$ derives from the

\footnotetext{
${ }^{5}$ We believe that this assumption is rather innocuous given that a supplier's pseudo-type is monotonically decreasing in his type. It may well be that it follows from the other properties, but this question is still open.
} 


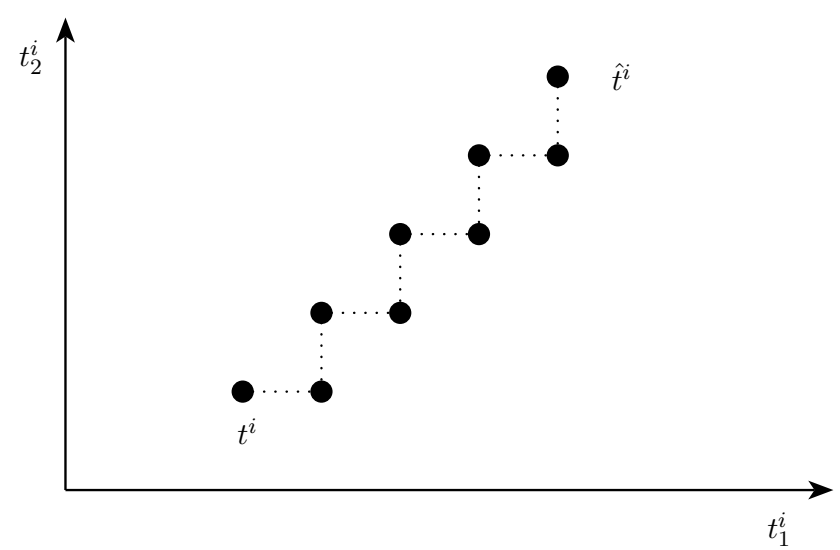

Figure 4.1: Moving from $t^{i}$ to $\hat{t}^{i}$ by only increasing one pseudo-type component at the time.

resulting outcome $(x(s), w(s))$ is

$$
u^{i}\left(s \mid \theta^{i}\right)=\sum_{j=1}^{d} x_{j}^{i}(s)\left(\max _{q_{j}}\left(\phi_{j}\left(q_{j}\right)-c_{j}^{i}\left(q_{j}, \theta^{i}\right)\right)-w_{j}^{i}(s)\right)-x_{0}^{i}(s) w_{0}^{i}(s) .
$$

Let us define $\tilde{x}^{i}(s)=\left(x_{1}^{i}(s), \ldots, x_{d}^{i}(s)\right)$, that is, $\tilde{x}^{i}(s)$ is the allocation vector $x^{i}(s)$ without the winning probability for the empty set $x_{0}^{i}(s)$. Now, using (4.5) the above can be written as

$$
u^{i}\left(s \mid \theta^{i}\right)=\tilde{x}^{i}(s) t^{i}\left(\theta^{i}\right)-x^{i}(s) w^{i}(s) .^{6}
$$

Notice that supplier $i$ 's pseudo-type captures his preference over outcomes $(x(),. w()$.$) . (As shown by$ Asker and Cantillon (2008), only quasi-linear scoring rules exhibit this property if suppliers' types are multi-dimensional.) By defining

$$
y^{i}(s)=x^{i}(s) w^{i}(s)
$$

we can simplify $(4.6)$ to

$$
u^{i}\left(s \mid \theta^{i}\right)=\tilde{x}^{i}(s) t^{i}\left(\theta^{i}\right)-y^{i}(s)
$$

If suppliers have the type profile $\theta$, the corresponding buyer utility for the outcome $(x(s), w(s))$

\footnotetext{
${ }^{6}$ Note that $\tilde{x}^{i}(s) t^{i}\left(\theta^{i}\right)$ and $x^{i}(s) w^{i}(s)$ are dot products. Other instances of dot products in the remainder of the chapter are not especially pointed out.
} 
is

$$
\begin{aligned}
u^{0}(s \mid \theta) & =\sum_{i=1}^{n} \sum_{j=1}^{d} x_{j}^{i}(s)\left(v_{j}\left(q_{j}^{* i}\left(\theta^{i}\right)\right)-\phi_{j}\left(q_{j}^{* i}\left(\theta^{i}\right)\right)+w_{j}^{i}(s)\right)+\sum_{i=1}^{n} x_{0}^{i}(s) w_{0}^{i}(s) \\
& =\sum_{i=1}^{n} \tilde{x}^{i}(s)\left(v\left(q^{* i}\left(\theta^{i}\right)\right)-\phi\left(q^{* i}\left(\theta^{i}\right)\right)\right)+x^{i}(s) w^{i}(s) \\
& =\sum_{i=1}^{n} \tilde{x}^{i}(s)\left(v\left(q^{* i}\left(\theta^{i}\right)\right)-\phi\left(q^{* i}\left(\theta^{i}\right)\right)\right)+y^{i}(s) .
\end{aligned}
$$

The first equality follows from the fact that the price supplier $i$ is asking for item set $j$ is determined by $\phi_{j}\left(q_{j}^{* i}\left(\theta^{i}\right)\right)-w_{j}^{i}(s)$ (see also $\left.(4.3)\right)$. The second equality follows from defining $v\left(q^{* i}\left(\theta^{i}\right)\right)=$ $\left(v_{1}\left(q_{1}^{* i}\left(\theta^{i}\right)\right), \ldots, v_{d}\left(q_{d}^{* i}\left(\theta^{i}\right)\right)\right)$ and a similar definition for $\phi\left(q^{* i}\left(\theta^{i}\right)\right)$. The last equality follows from (4.7).

Supplier $i$ 's bidding strategy $\beta^{i}$ maps each of his possible types $\theta^{i} \in \Theta^{i}$ into a price-quality bid $\left(p^{i}, q^{i}\right) \in \mathbb{R}_{+}^{d+m}$. Together with the scoring rule, $\beta^{i}$ implies a scored bidding strategy $b^{i}$ mapping each of $i$ 's types into a vector of scores $s^{i} \in \mathbb{R}^{d}$. Note that for the determination of outcomes (allocation vectors and winning scores), and hence the determination of the buyer's and suppliers' utilities, only the reported scores are of importance and not the price-quality bids that generated them. Therefore we concentrate in the following on scored bidding strategies. ${ }^{7}$

Consider supplier $i$ and assume that the other suppliers bid according to the profile of scored bidding strategies $b^{-i}=\left(b^{1}, \ldots, b^{i-1}, b^{i+1}, \ldots, b^{n}\right)$. If supplier $i$ has type $\theta^{i}$, then his expected utility for making a scored bid $s^{i}$ is

$$
\begin{aligned}
U^{i}\left(s^{i} \mid \theta^{i}\right) & =\int_{\Theta^{-i}}\left(\tilde{x}^{i}\left(s^{i}, b^{-i}\left(\theta^{-i}\right)\right) t^{i}\left(\theta^{i}\right)-y^{i}\left(s^{i}, b^{-i}\left(\theta^{-i}\right)\right)\right) \gamma^{-i}\left(\theta^{-i}\right) d \theta^{-i} \\
& =E_{-i}\left[\tilde{x}^{i}\left(s^{i}, b^{-i}\left(\theta^{-i}\right)\right) t^{i}\left(\theta^{i}\right)-y^{i}\left(s^{i}, b^{-i}\left(\theta^{-i}\right)\right)\right] .
\end{aligned}
$$

If suppliers bid according to the profile of scored bidding strategies $b=\left(b^{1}, \ldots, b^{n}\right)$, the expected utility of the buyer is

$$
U^{0}(b)=\int_{\theta \in \Theta^{n}}\left[\sum_{i=1}^{n} \tilde{x}^{i}(b(\theta))\left(v\left(q^{* i}\left(\theta^{i}\right)\right)-\phi\left(q^{* i}\left(\theta^{i}\right)\right)\right)+y^{i}(b(\theta))\right] \gamma(\theta) d \theta
$$

\footnotetext{
${ }^{7}$ The analysis done in the following section goes also through (in slightly adapted form) if one allows for mixed strategies, that is, $b^{i}$ maps each of supplier $i$ 's types into a distribution over finitely many score vectors. To simplify matters we stick to pure strategies.
} 
Definition 4.1 (Bayes-Nash Equilibrium) The profile of scored bidding strategies $b$ constitutes a Bayes-Nash equilibrium if for every supplier $i \in N$ and all types $\theta^{i} \in \Theta^{i}$ :

$$
\begin{array}{rlr} 
& E_{-i}\left[\tilde{x}^{i}\left(b^{i}\left(\theta^{i}\right), b^{-i}\left(\theta^{-i}\right)\right) t^{i}\left(\theta^{i}\right)-y^{i}\left(b^{i}\left(\theta^{i}\right), b^{-i}\left(\theta^{-i}\right)\right)\right] \\
\geq \quad & E_{-i}\left[\tilde{x}^{i}\left(s^{i}, b^{-i}\left(\theta^{-i}\right)\right) t^{i}\left(\theta^{i}\right)-y^{i}\left(s^{i}, b^{-i}\left(\theta^{-i}\right)\right)\right] \quad \forall s^{i} .
\end{array}
$$

Supplier $i$ 's scored bidding strategy might assign different bids to types yielding the same pseudotype. Given a profile of scored bidding strategies of the other suppliers, those bids either induce the same expected allocation vector for $i$ or differing expected allocation vectors. We conclude this section by formalizing this observation and introduce the following properties for suppliers' scored bidding strategies:

Definition 4.2 (Allocation Mixing) Consider the profile of scored bidding strategies b. Supplier $i$ 's strategy $b^{i}$ employs allocation mixing if there exist types $\theta^{i}, \hat{\theta}^{i} \in \Theta^{i}$ with $t^{i}\left(\theta^{i}\right)=t^{i}\left(\hat{\theta}^{i}\right)$ for which $b^{i}\left(\theta^{i}\right) \neq b^{i}\left(\hat{\theta}^{i}\right)$ and

$$
E_{-i}\left[\tilde{x}^{i}\left(b^{i}\left(\theta^{i}\right), b^{-i}\left(\theta^{-i}\right)\right)\right] \neq E_{-i}\left[\tilde{x}^{i}\left(b^{i}\left(\hat{\theta}^{i}\right), b^{-i}\left(\theta^{-i}\right)\right)\right]
$$

This property deals with the expected allocation vectors induced by bids from types yielding the same pseudo-type. Suppose that there are some types yielding the same pseudo-type for which supplier $i$ makes different scored bids. Allocation mixing means that, given a strategy profile $b^{-i}$ of the others, the expected allocation vectors for $i$ induced by those bids are not the same. Furthermore, we define

Definition 4.3 (Allocation Equivalent Mixing) Consider the profile of scored bidding strategies b. Supplier $i$ 's strategy $b^{i}$ employs allocation equivalent mixing if there exist types $\theta^{i}, \hat{\theta}^{i} \in \Theta^{i}$ with $t^{i}\left(\theta^{i}\right)=t^{i}\left(\hat{\theta}^{i}\right)$ for which $b^{i}\left(\theta^{i}\right) \neq b^{i}\left(\hat{\theta}^{i}\right)$ but

$$
E_{-i}\left[\tilde{x}^{i}\left(b^{i}\left(\theta^{i}\right), b^{-i}\left(\theta^{-i}\right)\right)\right]=E_{-i}\left[\tilde{x}^{i}\left(b^{i}\left(\hat{\theta}^{i}\right), b^{-i}\left(\theta^{-i}\right)\right)\right]
$$

Similar to the first property, this one also deals with the expected allocation vectors induced by bids from types yielding the same pseudo-type. Suppose that there are some types yielding the same pseudo-type for which supplier $i$ makes different scored bids. Allocation equivalent mixing means that, given a strategy profile $b^{-i}$ of the others, the expected allocation vectors for $i$ induced by those bids are the same.

In the next section we are going to establish a link between equilibria in the scoring auction which are based on types (that is, suppliers' bidding strategies can specify different bids for different types) 
and the ones which are based on pseudo-types (that is, suppliers' bidding strategies specify the same bid for all types yielding the same pseudo-type). For this we finally define

Definition 4.4 (Almost Everywhere (A.E.) Coinciding Strategies) We say that the profile of scored bidding strategies $b$ based on types coincides with the strategy profile $\hat{b}$ based on pseudo-types a.e. if $b$ and $\hat{b}$ differ only on a set of types with Lebesque measure zero.

\subsection{Pseudo-types Are Sufficient Statistics}

In this section we show the following: Suppose that every supplier's bidding strategy employs allocation equivalent mixing (see Definition 4.3) only for a set of types with Lebesgue measure zero. In order to analyze the set of possible equilibria in a scoring auction and the corresponding expected utilities of the buyer, it is then sufficient to consider a restricted setting in which suppliers' bids are based on their pseudo-types. That is, suppliers make the same scored bid for all types yielding the same pseudo-type. Thus, we show that pseudo-types are sufficient statistics, rendering suppliers original types redundant for the analysis of the scoring auction equilibria.

In a first step we show that every equilibrium in the scoring auction is outcome equivalent to an auxiliary, mixed strategy equilibrium. In this auxiliary equilibrium each supplier $i$ associates all types yielding the same pseudo-type with the same mixed bid. Two equilibria are outcome equivalent if they both induce the same distribution over outcomes (that is allocation vectors and winning scores).

Lemma 4.1 For every equilibrium $b$ in the scoring auction there exists an outcome equivalent mixed strategy equilibrium $\tilde{b}$ such that $\tilde{b}^{i}\left(\theta^{i}\right)=\tilde{b}^{i}\left(\hat{\theta}^{i}\right)$ whenever $t^{i}\left(\theta^{i}\right)=t^{i}\left(\hat{\theta}^{i}\right)$.

\section{Proof}

Let $b=\left(b^{1} \ldots, b^{n}\right)$ be an equilibrium in the scoring auction. For each supplier $i$ and each pseudotype $t^{i} \in T^{i}$ consider the mixed bid $r^{i}\left(t^{i}\right)$ mapping $t^{i}$ into a distribution over vectors of scores. The support of $r^{i}\left(t^{i}\right)$ consists of the different scored bids generated by $b^{i}$ for all the types yielding pseudo-type $t^{i}$. That is, the support set is $\left\{b^{i}\left(\theta^{i}\right) \mid \theta^{i} \in \Theta_{t^{i}}^{i}\right\}$. Each scored bid in the support of $r^{i}\left(t^{i}\right)$ is played with the relative frequency with which it is played by all the types in $\Theta_{t^{i}}^{i}$. More precisely, some scored bid $s^{i}$ in the support of $r^{i}\left(t^{i}\right)$ is played with probability

$$
\int_{\theta^{i} \in \Theta_{t^{i}}^{i}} \delta_{b^{i}\left(\theta^{i}\right)} \gamma^{i}\left(\theta^{i} \mid \Theta_{t^{i}}^{i}\right),
$$


where $\gamma^{i}\left(. \mid \Theta_{t^{i}}^{i}\right)$ denotes the conditional type distribution on $\Theta_{t^{i}}^{i}$ and $\delta_{b^{i}\left(\theta^{i}\right)}$ is defined as follows

$$
\delta_{b^{i}\left(\theta^{i}\right)}=\left\{\begin{array}{ll}
1 & \text { if } b^{i}\left(\theta^{i}\right)=s^{i} \\
0 & \text { otherwise }
\end{array} .\right.
$$

Now we can construct a new equilibrium in which each supplier $i$ has the same bidding strategy for all types yielding the same pseudo-type. This is done by assigning the same mixed bid $r^{i}\left(t^{i}\right)$ to all $\theta^{i} \in \Theta_{t^{i}}^{i}$. That is, for all $\theta^{i} \in \Theta^{i}$ we define $\tilde{b}^{i}\left(\theta^{i}\right)=r^{i}\left(t^{i}\left(\theta^{i}\right)\right)$.

That $\tilde{b}=\left(\tilde{b}^{1}, \ldots, \tilde{b}^{n}\right)$ is indeed an equilibrium can be seen as follows: First, note that by construction the distribution of bids coming from each supplier $i$ remains unchanged when he switches from $b^{i}$ to $\tilde{b}^{i}$. Second, consider supplier $i$ 's expected utility. Since $b$ is an equilibrium, we have by Definition 4.1 that for all $\theta^{i} \in \Theta^{i}$,

$$
b^{i}\left(\theta^{i}\right) \in \arg \max _{s^{i}} E_{-i}\left[\tilde{x}^{i}\left(s^{i}, b^{-i}\left(\theta^{-i}\right)\right) t^{i}\left(\theta^{i}\right)-y^{i}\left(s^{i}, b^{-i}\left(\theta^{-i}\right)\right)\right] .
$$

Since supplier $i$ 's private information enters his expected utility only via his pseudo-type, he is indifferent about the bids adapted by all the types yielding the same pseudo-type. That is, the bid $b^{i}\left(\theta^{i}\right)$ is also a best reply for all other $\hat{\theta}^{i} \in \Theta_{t^{i}\left(\theta^{i}\right)}^{i}$. Hence, every bid in the support of $\tilde{b}^{i}\left(\theta^{i}\right)$ is a best reply for all $\hat{\theta}^{i} \in \Theta_{t^{i}\left(\theta^{i}\right)}^{i}$. It follows that $\tilde{b}^{i}$ is a best response for supplier $i$ against $b^{-i}$. As mentioned before, the distribution of bids coming from the other suppliers is not changing when switching from $b^{-i}$ to $\tilde{b}^{-i}$. Thus, $\tilde{b}^{i}$ is also a best response against $\tilde{b}^{-i}$, and $\tilde{b}$ is indeed an equilibrium. Furthermore, $b$ and $\tilde{b}$ are outcome equivalent as both equilibria induce the same distribution over scored bids and therefore also the same distribution over outcomes.

Above we have seen that the suppliers are indifferent between an equilibrium $b$ and its outcome equivalent, mixed strategy counterpart $\tilde{b}$ (constructed as in the proof of Lemma 4.1). Their expected utilities are the same in both. The buyer is also indifferent between these two equilibria. However, this cannot as easily be observed as in the suppliers' case. In order to show this, we have to make use of one of the following lemmas, more specifically Lemma 4.3. The details are given in Section 4.4. Asker and Cantillon (2008) illustrate this difficulty for a special case of our model in which only one item is to be allocated among the suppliers.

Now, let us inspect the auxiliary equilibrium $\tilde{b}$ constructed in Lemma 4.1 a bit further. Remember that in $\tilde{b}^{i}$ all types yielding the same pseudo-type $t^{i}$ have the same mixed bid $r^{i}\left(t^{i}\right)$. By $h^{i}$ we denote 
a selection from the support of these mixed bids. That is,

$$
h^{i}\left(t^{i}\right) \in \operatorname{supp} r^{i}\left(t^{i}\right) \quad \forall t^{i} \in T^{i} .
$$

Considering two pseudo-types $t^{i}, \hat{t}^{i} \in T^{i}$, we can observe that ${ }^{8}$

$$
\begin{aligned}
& E_{-i}\left[\tilde{x}^{i}\left(h^{i}\left(t^{i}\right), \tilde{b}^{-i}\left(\theta^{-i}\right)\right) t^{i}-y^{i}\left(h^{i}\left(t^{i}\right), \tilde{b}^{-i}\left(\theta^{-i}\right)\right)\right] \\
\geq & E_{-i}\left[\tilde{x}^{i}\left(h^{i}\left(\hat{t}^{i}\right), \tilde{b}^{-i}\left(\theta^{-i}\right)\right) t^{i}-y^{i}\left(h^{i}\left(\hat{t}^{i}\right), \tilde{b}^{-i}\left(\theta^{-i}\right)\right)\right] .
\end{aligned}
$$

The above follows from $\tilde{b}$ being an equilibrium and every element in the support of $r^{i}\left(t^{i}\right)$ being a best response against $\tilde{b}^{-i}$ for all types $\theta^{i} \in \Theta_{t^{i}}^{i}$ (see also explanation in the proof of Lemma 4.1). Similarly, we also have that

$$
\begin{aligned}
& E_{-i}\left[\tilde{x}^{i}\left(h^{i}\left(\hat{t}^{i}\right), \tilde{b}^{-i}\left(\theta^{-i}\right)\right) \hat{t}^{i}-y^{i}\left(h^{i}\left(\hat{t}^{i}\right), \tilde{b}^{-i}\left(\theta^{-i}\right)\right)\right] \\
\geq & E_{-i}\left[\tilde{x}^{i}\left(h^{i}\left(t^{i}\right), \tilde{b}^{-i}\left(\theta^{-i}\right)\right) \hat{t}^{i}-y^{i}\left(h^{i}\left(t^{i}\right), \tilde{b}^{-i}\left(\theta^{-i}\right)\right)\right] .
\end{aligned}
$$

By adding (4.13) and (4.14) we get the following monotonicity condition,

$$
\left(E_{-i}\left[\tilde{x}^{i}\left(h^{i}\left(t^{i}\right), \tilde{b}^{-i}\left(\theta^{-i}\right)\right)\right]-E_{-i}\left[\tilde{x}^{i}\left(h^{i}\left(\hat{t}^{i}\right), \tilde{b}^{-i}\left(\theta^{-i}\right)\right)\right]\right)\left(t^{i}-\hat{t}^{i}\right) \geq 0 .
$$

Note that in order to derive the above, we employed the very same steps as in the derivation of the monotonicity conditions in the foregoing chapters. (Check the passages leading up to Definitions 2.2 and 3.2 for details.)

Using (4.15) we can establish our next result. Specifically, we show that for any selection $h^{i}$, supplier $i$ 's expected allocation vector $E_{-i}\left[\tilde{x}^{i}\left(h^{i}(),. \tilde{b}^{-i}\left(\theta^{-i}\right)\right)\right]$ is componentwise nondecreasing. In the following $\dot{\leq}$ denotes "componentwise smaller or equal".

Lemma 4.2 Consider the equilibrium $\tilde{b}$ constructed in Lemma 4.1 where every type yielding pseudotype $t^{i}$ has the same mixed bid $r^{i}\left(t^{i}\right)$. Let $h^{i}$ be some selection from $r^{i}($.$) (see also (4.12)). For every$ supplier $i$ and all $t^{i}, \hat{t}^{i} \in T^{i}$ :

$$
\text { If } t^{i} \dot{\leq} \hat{t}^{i} \text { then } E_{-i}\left[\tilde{x}^{i}\left(h^{i}\left(t^{i}\right), \tilde{b}^{-i}\left(\theta^{-i}\right)\right)\right] \dot{\leq} E_{-i}\left[\tilde{x}^{i}\left(h^{i}\left(\hat{t}^{i}\right), \tilde{b}^{-i}\left(\theta^{-i}\right)\right)\right]
$$

\footnotetext{
${ }^{8}$ Generally, $E_{-i}\left[\tilde{x}^{i}\left(s^{i}, \tilde{b}^{-i}\left(\theta^{-i}\right)\right)\right]$ is the expected allocation vector for supplier $i$ if he makes a scored bid $s^{i}$ and the other suppliers bid according to $\tilde{b}^{-i}$. Given that $\tilde{b}^{-i}$ is a profile of mixed bidding strategies, note that in this case $E_{-i}[$. denotes the expectation taken over all type profiles $\theta^{-i}$ as well as the corresponding mixed strategies $\tilde{b}^{-i}\left(\theta^{-i}\right)$. The same goes for $E_{-i}\left[y^{i}\left(s^{i}, \tilde{b}^{-i}\left(\theta^{-i}\right)\right)\right]$. Also, note that (4.13) is not generally valid for equilibrium bidding strategies that involve continuous mixed bids. In our case it is valid because of the special way we constructed $\tilde{b}$.
} 


\section{Proof}

Suppose that $b=\left(b^{1}, \ldots, b^{n}\right)$ is an equilibrium in the scoring auction and that $\tilde{b}=\left(\tilde{b}^{1}, \ldots, \tilde{b}^{n}\right)$ is the corresponding auxiliary, mixed strategy equilibrium constructed in Lemma 4.1. Take some supplier $i$ and some pseudo-types $t^{i}, \hat{t}^{i} \in T^{i}$ with $t^{i} \dot{\leq} \hat{t}^{i}$. Note that in $\tilde{b}^{i}$ all of supplier $i$ 's types yielding the same pseudo-type $t^{i}$ are associated with the same mixed bid $r^{i}\left(t^{i}\right)$. Now we pick some selection $h^{i}$ from the support of $r^{i}($.$) , see also (4.12).$

Via a sequence of pseudo-types $\left\{\tilde{t}^{i, 0}=t^{i}, \tilde{t}^{i, 1}, \ldots, \tilde{t}^{i, k}, \tilde{t}^{i, k+1}=\hat{t}^{i}\right\}$ we now move from $t^{i}$ to $\hat{t}^{i}$. The elements of this sequence are picked such that moving from one element to the next one requires only a one-dimensional increase. ${ }^{9}$ Figure 4.2 illustrates this for a simple two-dimensional example.

So, with $\tilde{t}^{i, 0}$ as the starting point, we move on to $\tilde{t}^{i, 1}$ by only changing the $j^{*}$ 's element. Thus, by construction we have that $\tilde{t}_{j^{*}}^{i, 0} \neq \tilde{t}_{j^{*}}^{i, 1}$, whereas $\tilde{t}_{j}^{i, 0}=\tilde{t}_{j}^{i, 1}$ for all the remaining $j \neq j^{*}$. From (4.15) it follows that

$$
\left(E_{-i}\left[\tilde{x}^{i}\left(h^{i}\left(\tilde{t}^{i, 1}\right), \tilde{b}^{-i}\left(\theta^{-i}\right)\right)\right]-E_{-i}\left[\tilde{x}^{i}\left(h^{i}\left(\tilde{t}^{i, 0}\right), \tilde{b}^{-i}\left(\theta^{-i}\right)\right)\right]\right)\left(\tilde{t}^{i, 1}-\tilde{t}^{i, 0}\right) \geq 0 .
$$

Since only $\tilde{t}_{j^{*}}^{i, 1}$ differs from $\tilde{t}_{j^{*}}^{i, 0}$, whereas all the other elements of the two pseudo-types are the same, the above inequality becomes

$$
\left(E_{-i}\left[\tilde{x}^{i}\left(h^{i}\left(\tilde{t}^{i, 1}\right), \tilde{b}^{-i}\left(\theta^{-i}\right)\right)\right]_{j^{*}}-E_{-i}\left[\tilde{x}^{i}\left(h^{i}\left(\tilde{t}^{i, 0}\right), \tilde{b}^{-i}\left(\theta^{-i}\right)\right)\right]_{j^{*}}\right)\left(\tilde{t}_{j^{*}}^{i, 1}-\tilde{t}_{j^{*}}^{i, 0}\right) \geq 0,
$$

where $E_{-i}\left[\tilde{x}^{i}\left(., \tilde{b}^{-i}\left(\theta^{-i}\right)\right)\right]_{j^{*}}$ signifies the $j^{*}$ 's element of supplier $i$ 's expected allocation vector. Thus, if the pseudo-type increases in the $j^{*}$ 's dimension then the expected allocation vector also weakly increases in this dimension.

Now, we repeat the above steps while moving from $\tilde{t}^{i}, 1$ to $\hat{t}^{i}$ along the remaining elements of the pseudo-type sequence. Using this procedure we find that

$$
E_{-i}\left[\tilde{x}^{i}\left(h^{i}\left(t^{i}\right), \tilde{b}^{-i}\left(\theta^{-i}\right)\right)\right] \dot{\leq} E_{-i}\left[\tilde{x}^{i}\left(h^{i}\left(\hat{t}^{i}\right), \tilde{b}^{-i}\left(\theta^{-i}\right)\right)\right] .
$$

\footnotetext{
${ }^{9}$ One of the assumptions made in Section 4.2.2 is that the pseudo-type space is rich enough to ensure the existence of such a sequence.
} 


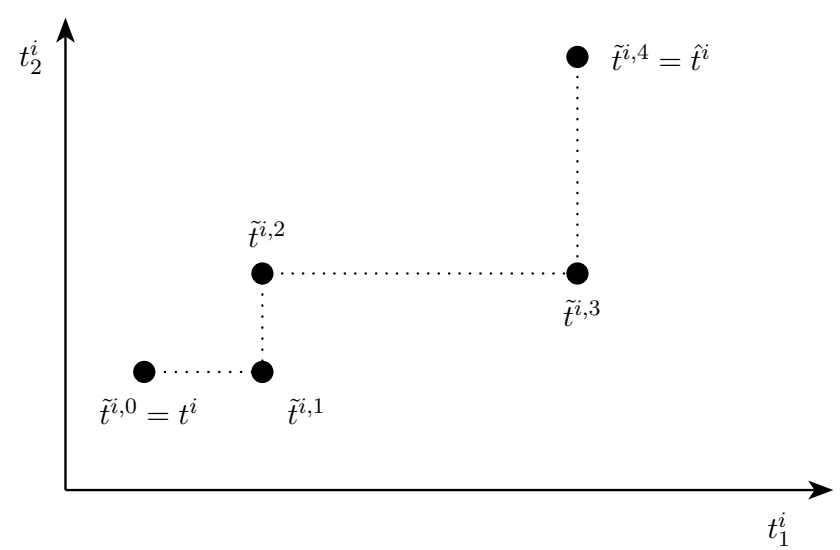

Figure 4.2: Moving from $t^{i}$ to $\hat{t}^{i}$ by only increasing one dimension of the pseudo-type at the time.

Next, we show that for each supplier $i, r^{i}($.$) is not mixing over scored bids which induce different$ expected allocations for $i$, except possibly on a set of measure zero. In order to do so we employ the following theorem of Lavrič (1993).

Theorem 4.1 (Lavrič (1993)) Let $A$ be a nonempty subset of $\mathbb{R}^{n}$ and let $f: A \mapsto \mathbb{R}^{m}$ be a componentwise nondecreasing or nonincreasing function. Then the set of all points of discontinuity of $f$ is of Lebesgue measure zero.

Using the above result we can now state the following:

Lemma 4.3 Consider the equilibrium $\tilde{b}$ constructed in Lemma 4.1 where every type yielding pseudotype $t^{i}$ has the same mixed bid $r^{i}\left(t^{i}\right)$. For each supplier $i$, the set of pseudo-types $t^{i} \in T^{i}$ for which there exist elements $s^{i}, \hat{s}^{i}$ in the support of $r^{i}\left(t^{i}\right)$ such that

$$
E_{-i}\left[\tilde{x}^{i}\left(s^{i}, \tilde{b}^{-i}\left(\theta^{-i}\right)\right)\right] \neq E_{-i}\left[\tilde{x}^{i}\left(\hat{s}^{i}, \tilde{b}^{-i}\left(\theta^{-i}\right)\right)\right]
$$

has Lebesgue measure zero.

\section{Proof}

Suppose that $b=\left(b^{1}, \ldots, b^{n}\right)$ is an equilibrium in the scoring auction and that $\tilde{b}=\left(\tilde{b}^{1}, \ldots, \tilde{b}^{n}\right)$ is the corresponding auxiliary, mixed strategy equilibrium constructed in Lemma 4.1. Take some supplier $i$ and pick two selections $h^{i 1}, h^{i 2}$ from the support of $r^{i}\left(\right.$.) (see (4.12)). Take $t^{i} \in T^{i}$ and consider a sequence of pseudo-types $\left(t^{i k}\right)_{k \in \mathbb{N}}$ in $T^{i}$ with $\lim _{k \rightarrow \infty}\left(t^{i k}\right)=t^{i}$. More specifically, we choose the sequence such that every element is componentwise smaller than $t^{i}$. That is, $t^{i k} \dot{\leq} t^{i}$ for all $k$. (If $t^{i}$ 
already constitutes the lowest possible pseudo-type, one can do the following analysis by choosing a sequence in which every element is componentwise bigger than $t^{i}$.)

First of all, Lemma 4.2 implies that ${ }^{10}$

$$
E_{-i}\left[\tilde{x}^{i}\left(h^{i 1}\left(t^{i k}\right), \tilde{b}^{-i}\left(\theta^{-i}\right)\right)\right] \dot{\leq} E_{-i}\left[\tilde{x}^{i}\left(h^{i 2}\left(t^{i}\right), \tilde{b}^{-i}\left(\theta^{-i}\right)\right)\right] \quad \forall t^{i k}
$$

Furthermore, from Lemma 4.2 in conjunction with Lavrič's (1993) Theorem 4.1it follows that $E_{-i}\left[\tilde{x}^{i}\left(h^{i 1}(),. \tilde{b}^{-i}\left(\theta^{-i}\right)\right)\right]$ is continuous almost everywhere. Thus,

$$
E_{-i}\left[\tilde{x}^{i}\left(h^{i 1}\left(t^{i k}\right), \tilde{b}^{-i}\left(\theta^{-i}\right)\right)\right] \rightarrow E_{-i}\left[\tilde{x}^{i}\left(h^{i 1}\left(t^{i}\right), \tilde{b}^{-i}\left(\theta^{-i}\right)\right)\right] \quad \text { a.e. }
$$

as $t^{i k} \rightarrow t^{i}$. From the above it follows that

$$
E_{-i}\left[\tilde{x}^{i}\left(h^{i 1}\left(t^{i}\right), \tilde{b}^{-i}\left(\theta^{-i}\right)\right)\right] \dot{\leq} E_{-i}\left[\tilde{x}^{i}\left(h^{i 2}\left(t^{i}\right), \tilde{b}^{-i}\left(\theta^{-i}\right)\right)\right] \quad \text { a.e. }
$$

Similarly, Lemma 4.2 also implies that ${ }^{11}$

$$
E_{-i}\left[\tilde{x}^{i}\left(h^{i 2}\left(t^{i k}\right), \tilde{b}^{-i}\left(\theta^{-i}\right)\right)\right] \dot{\leq} E_{-i}\left[\tilde{x}^{i}\left(h^{i 1}\left(t^{i}\right), \tilde{b}^{-i}\left(\theta^{-i}\right)\right)\right] \quad \forall t^{i k}
$$

Once more, from Lemma 4.2 in conjunction with Lavrič's (1993) Theorem 4.1 it follows that $E_{-i}\left[\tilde{x}^{i}\left(h^{i 2}(),. \tilde{b}^{-i}\left(\theta^{-i}\right)\right)\right]$ is continuous almost everywhere. Thus,

$$
E_{-i}\left[\tilde{x}^{i}\left(h^{i 2}\left(t^{i k}\right), \tilde{b}^{-i}\left(\theta^{-i}\right)\right)\right] \rightarrow E_{-i}\left[\tilde{x}^{i}\left(h^{i 2}\left(t^{i}\right), \tilde{b}^{-i}\left(\theta^{-i}\right)\right)\right] \quad \text { a.e. }
$$

as $t^{i k} \rightarrow t^{i}$. From the above it follows that

$$
E_{-i}\left[\tilde{x}^{i}\left(h^{i 2}\left(t^{i}\right), \tilde{b}^{-i}\left(\theta^{-i}\right)\right)\right] \dot{\leq} E_{-i}\left[\tilde{x}^{i}\left(h^{i 1}\left(t^{i}\right), \tilde{b}^{-i}\left(\theta^{-i}\right)\right)\right] \quad \text { a.e. }
$$

\footnotetext{
${ }^{10}$ In order to clarify this step, consider the following selection $h^{i}$ in Lemma 4.2:

$$
h^{i}\left(t^{* i}\right)=\left\{\begin{array}{ll}
h^{i 1}\left(t^{* i}\right) & \text { if } t^{* i} \neq t^{i} \\
h^{i 2}\left(t^{* i}\right) & \text { if } t^{* i}=t^{i}
\end{array} .\right.
$$

${ }^{11}$ Again, in order to clarify this step, consider the following selection $h^{i}$ in Lemma 4.2:

$$
h^{i}\left(t^{* i}\right)=\left\{\begin{array}{ll}
h^{i 1}\left(t^{* i}\right) & \text { if } t^{* i}=t^{i} \\
h^{i 2}\left(t^{* i}\right) & \text { if } t^{* i} \neq t^{i}
\end{array} .\right.
$$
}


Finally, (4.16) and (4.17) together imply that

$$
E_{-i}\left[\tilde{x}^{i}\left(h^{i 1}\left(t^{i}\right), \tilde{b}^{-i}\left(\theta^{-i}\right)\right)\right]=E_{-i}\left[\tilde{x}^{i}\left(h^{i 2}\left(t^{i}\right), \tilde{b}^{-i}\left(\theta^{-i}\right)\right)\right] \quad \text { a.e. }
$$

Thus, the set of pseudo-types $t^{i}$ for which there exist elements $s^{i}, \hat{s}^{i}$ in the support of $r^{i}\left(t^{i}\right)$ such that $E_{-i}\left[\tilde{x}^{i}\left(s^{i}, \tilde{b}^{-i}\left(\theta^{-i}\right)\right)\right] \neq E_{-i}\left[\tilde{x}^{i}\left(\hat{s}^{i}, \tilde{b}^{-i}\left(\theta^{-i}\right)\right)\right]$ has Lebesgue measure zero.

As we have already seen before, the suppliers are indifferent between an equilibrium $b$ and its outcome equivalent, mixed counterpart $\tilde{b}$. Their expected utilities are the same in both equilibria. Utilizing the above Lemma 4.3, we can now claim the same for the buyer. The details for this can be found in Section 4.4.

Considering the auxiliary equilibrium bidding strategy $\tilde{b}^{i}$, recall that the support of the mixed bid $r^{i}\left(t^{i}\right)$ consists of the different scored bids generated by the original equilibrium strategy $b^{i}$ for all the types in $\Theta_{t^{i}}^{i}$. Suppose that for some pseudo-type $t^{i}$ there exist scored bids in the support of $r^{i}\left(t^{i}\right)$ which yield different expected allocation vectors for $i$. This means that $b^{i}$ employs allocation mixing (see Definition 4.2 in the foregoing section) for the types in $\Theta_{t^{i}}^{i}$. Based on this observation we find the following result:

Lemma 4.4 In every equilibrium $b$ in the scoring auction, supplier $i$ 's bidding strategy $b^{i}$ employs allocation mixing only for a set of types $\theta^{i} \in \Theta^{i}$ that has Lebesgue measure zero.

\section{Proof}

Suppose that $b$ is an equilibrium in the scoring auction. Take some pseudo-type $t^{i} \in T^{i}$ and consider all the types yielding this pseudo-type. If there exist $\theta^{i}, \hat{\theta}^{i} \in \Theta_{t^{i}}^{i}$ such that $E_{-i}\left[\tilde{x}^{i}\left(b^{i}\left(\theta^{i}\right), b^{-i}\left(\theta^{-i}\right)\right)\right] \neq$ $E_{-i}\left[\tilde{x}^{i}\left(b^{i}\left(\hat{\theta}^{i}\right), b^{-i}\left(\theta^{-i}\right)\right)\right]$ then $b^{i}$ employs allocation mixing for $\Theta_{t^{i}}^{i}$. From Lemma 4.3 it follows that the set of pseudo-types for which this happens has Lebesgue measure zero. This implies that also the corresponding set of types yielding these pseudo-types has Lebesgue measure zero. (Remember from the foregoing section that one property of the pseudo-type mapping is as follows: If a set of pseudo-types has Lebesgue measure zero then the set of types yielding this set of pseudo-types also has Lebesgue measure zero.)

Let us restrict our attention to the class of equilibria in the scoring auction where suppliers employ allocation equivalent mixing only for a zero measure of types. Using Lemma 4.4 we can now state the following main result of this section: 
Theorem 4.2 Suppose that the set of types for which suppliers employ allocation equivalent mixing in their bidding strategies has Lebesgue measure zero. Then, for every equilibrium in the scoring auction based on types there exists a pseudo-type based equilibrium that coincides with it a.e. (see Definition 4.4). Vice versa, every equilibrium in the restricted setting, where strategies are based on pseudo-types, is also an equilibrium in the unrestricted setting.

\section{Proof}

Trivially, all equilibria in the scoring auction where suppliers are constrained to make the same bid for all types yielding the same pseudo-type are also equilibria in the unconstrained scoring auction.

Now let us consider the opposite direction. Suppose that suppliers employ allocation equivalent mixing in their biding strategies only for a zero measure of types. Furthermore, suppose that $b$ is an equilibrium in the unconstrained scoring auction. Based on $b$ we can construct an equilibrium $\hat{b}$ in which suppliers make the same bid for types yielding the same pseudo-type and which differs from $b$ at most on a set of measure zero. This is done as follows: For every supplier $i$ and every $t^{i} \in T^{i}$ we pick some $\bar{\theta}^{i} \in \Theta_{t^{i}}^{i}$ and set $\hat{b}^{i}\left(\theta^{i}\right)=b^{i}\left(\bar{\theta}^{i}\right)$ for all $\theta^{i} \in \Theta_{t^{i}}^{i}$. As already pointed out before, supplier $i$ 's private information enters his expected utility only via his pseudo-type. So, $\hat{b}^{i}$ is also best response against $b^{-i}$ (see argument in the proof of Lemma 4.1). From Lemma 4.4 it follows that for every supplier $i, \hat{b}^{i}$ differs from $b^{i}$ only on a set of types with Lebesgue measure zero. Hence, $\hat{b}^{i}$ is also a best response against $\hat{b}^{-i}$.

Considering the above proof, note that supplier $i$ switching from his original, unconstrained scored bidding strategy $b^{i}$ to the constrained one, $\hat{b}^{i}$, is inconsequential to his own expected utility. This is because his private information enters his expected utility only via the pseudo-type (again, see the argument in the proof of Lemma 4.1). However, if $\hat{b}^{i}$ differs from $b^{i}$ on a non-zero measure of types then we cannot guarantee that the strategy profile $b^{-i}$ (and hence also $\hat{b}^{-i}$ ) is still a best reply for the other suppliers. ${ }^{12}$ We can ensure that $\hat{b}^{i}$ differs from $b^{i}$ only on a zero measure of types, if $b^{i}$

\footnotetext{
${ }^{12}$ In order to illustrate this point consider the following simple game. There are only two players and each player has two possible actions. Player 1 has action set $\{U, D\}$, and player 2 's action set is $\{L, R\}$. The resulting utilities for both players are as follows:

\begin{tabular}{c|c|c|}
\multicolumn{1}{c}{$L$} & \multicolumn{1}{c}{$R$} \\
\cline { 2 - 3 }$U$ & 0,2 & 0,1 \\
\cline { 2 - 3 }$D$ & 0,0 & 0,1 \\
\cline { 2 - 3 } & &
\end{tabular}

Notice that, no matter what player 2 does, player 1's utility is always 0 . One can easily see that for example player 1 playing $U$ with probability $\frac{1}{3}$ and $D$ with probability $\frac{2}{3}$ and player 2 playing $R$ is an equilibrium. Suppose player 1 switches to playing $U$ with probability 1 . This move is inconsequential to his own expected utility. However, player 2 could now increase his expected utility by switching from playing $R$ to playing $L$.
} 
employs allocation mixing and allocation equivalent mixing only for a zero measure of types. With respect to allocation mixing we can verify this analytically (see Lemma 4.4). However, for allocation equivalent mixing we cannot do this and have to exclude it by assumption.

Thus, considering the analysis of the set of possible equilibria in a scoring auction, we can confine ourselves to a restricted setting where suppliers bid solely based on their pseudo-types. Note that the expected utilities of the buyer and the suppliers are the same in a type based equilibrium and in the corresponding pseudo-type based equilibrium that coincides with it almost everywhere (constructed as in the proof of Theorem 4.2). For details see

We conclude this section by pointing out the link between Theorem 4.2 and the main result of Asker and Cantillon (2008, Theorem 1). Suppliers' equilibrium bidding strategies, mapping their possible types into vectors of scores, together with the allocation rule and the winning score rule imply an equilibrium outcome function which maps suppliers' possible types into outcomes $(x(),. y()$.$) . From$ Theorem 4.2 it readily follows that for every equilibrium in the scoring auction based on types, there exists an equilibrium based on pseudo-types which induces the same equilibrium outcome function, except possibly on a set of types with Lebesgue measure zero. (Again, this result is of course based on the assumption that suppliers essentially do not employ allocation equivalent mixing.) This finding is the direct combinatorial scoring auction extension of Asker and Cantillon's (2008) main result about the role of pseudo-types as sufficient statistics in single item scoring auctions (their Theorem 1). However, their result is stated in terms of equilibrium outcome functions rather than in terms of the underlying equilibrium bidding strategies (like our Theorem 4.2).

Similar to Asker and Cantillon (2008) we use two main facts in order to establish Lemma 4.4 (stating that the set of types for which suppliers' equilibrium strategies employ allocation mixing has zero measure). First, we construct for every equilibrium in the scoring auction an auxiliary, mixed strategy equilibrium (see Lemma 4.1). We do this in the same way as Asker and Cantillon (2008). Suppliers' bidding strategies in this auxiliary equilibrium are based on their pseudo-types. This equilibrium is constructed in such a way that a supplier's equilibrium bidding strategy specifies a mixed bid for each of his pseudo-types. Considering a specific pseudo-type $t^{i}$, the support of such a mixed bid consists of all the pure bids that the original equilibrium bidding function specifies for the types yielding $t^{i}$ as the pseudo-type. Second, we prove that for almost all pseudo-types this support does not contain pure bids yielding different expected allocations for the supplier (see Lemma 4.3). In order to establish this result we use a different method than Asker and Cantillon (2008). Our method highlights the link to the monotonicity conditions used in the foregoing chapters (see (4.15) for details). 


\subsection{Technical Note Concerning the Buyer's Utility}

Let $b=\left(b^{1}, \ldots, b^{n}\right)$ be an equilibrium in the scoring auction. In Lemma 4.1 we have constructed a new mixed strategy equilibrium $\tilde{b}$ which is outcome equivalent to $b$. That is, $\tilde{b}$ induces the same distribution over scored bids as $b$. Hence, it also induces the same distribution over outcomes. As pointed out before, it can be easily observed that suppliers are indifferent between $b$ and $\tilde{b}$ as their expected utilities are the same in both. The same can be claimed for the buyer, however the argument is not as straightforward as in the suppliers' case. The reason is that for the buyer's expected utility not only the overall distribution of outcomes is of interest, but also the question which outcomes are associated with which type-realizations of the suppliers. To illustrate this point we start by considering a simplified setting where we only change the bidding strategy of one supplier.

Consider $\hat{b}=\left(\hat{b}^{1}, b^{2}, \ldots, b^{n}\right)$ where $\hat{b}^{1}$ is constructed based on $b^{1}$ as described in the proof to Lemma 4.1. By the logic described in this proof, $\hat{b}$ is an outcome equivalent equilibrium to $b$. If suppliers bid according to $b$, the expected utility of the buyer (given in (4.11)) can be decomposed into two terms. Specifically,

$$
\begin{aligned}
U^{0}(b)= & \int_{\theta \in \Theta}\left[\sum_{i=1}^{n} \tilde{x}^{i}(b(\theta))\left(v\left(q^{* i}\left(\theta^{i}\right)\right)-\phi\left(q^{* i}\left(\theta^{i}\right)\right)\right)+y^{i}(b(\theta))\right] \gamma(\theta) d \theta \\
= & \int_{\theta \in \Theta}\left[\sum_{i=2}^{n} \tilde{x}^{i}(b(\theta))\left(v\left(q^{* i}\left(\theta^{i}\right)\right)-\phi\left(q^{* i}\left(\theta^{i}\right)\right)\right)+y^{i}(b(\theta))\right] \gamma(\theta) d \theta \\
& +\int_{\theta \in \Theta}\left[\tilde{x}^{1}(b(\theta))\left(v\left(q^{* 1}\left(\theta^{1}\right)\right)-\phi\left(q^{* 1}\left(\theta^{1}\right)\right)\right)+y^{1}(b(\theta))\right] \gamma(\theta) d \theta
\end{aligned}
$$

Now, let us consider the first term in (4.18). Take some $i \in\{2, \ldots, n\}$ and look at

$$
\begin{aligned}
& \int_{\theta \in \Theta}\left[\tilde{x}^{i}(b(\theta))\left(v\left(q^{* i}\left(\theta^{i}\right)\right)-\phi\left(q^{* i}\left(\theta^{i}\right)\right)\right)+y^{i}(b(\theta))\right] \gamma(\theta) d \theta \\
= & \int_{\theta^{i} \in \Theta^{i}}\left[E_{-i}\left[\tilde{x}^{i}\left(b^{i}\left(\theta^{i}\right), b^{-i}\left(\theta^{-i}\right)\right)\right]\left(v\left(q^{* i}\left(\theta^{i}\right)\right)-\phi\left(q^{* i}\left(\theta^{i}\right)\right)\right)\right] \gamma^{i}\left(\theta^{i}\right) d \theta^{i} \\
& +\int_{\theta \in \Theta}\left[y^{i}(b(\theta))\right] \gamma(\theta) d \theta .
\end{aligned}
$$

Since by construction the distribution of suppliers' bids under $b$ is the same as under $\hat{b}$, the second 
term of (4.19) is not changing if we switch from equilibrium $b$ to $\hat{b}$. That is, ${ }^{13}$

$$
\int_{\theta \in \Theta}\left[y^{i}(b(\theta))\right] \gamma(\theta) d \theta=\int_{\theta \in \Theta}\left[y^{i}(\hat{b}(\theta))\right] \gamma(\theta) d \theta
$$

Similarly, since by construction $\hat{b}^{i}=b^{i}$ and the distribution of bids coming from the other suppliers is the same under $b$ and $\hat{b}$, we find that for all $\theta^{i} \in \Theta^{i}$

$$
E_{-i}\left[\tilde{x}^{i}\left(b^{i}\left(\theta^{i}\right), b^{-i}\left(\theta^{-i}\right)\right)\right]=E_{-i}\left[\tilde{x}^{i}\left(\hat{b}^{i}\left(\theta^{i}\right), \hat{b}^{-i}\left(\theta^{-i}\right)\right)\right] .
$$

Thus, the first term of (4.19) is also not changing if we switch from $b$ to $\hat{b}^{14}$ Hence, we find that overall the first term in (4.18) is not changing if we switch from $b$ to $\hat{b}$.

Now, let us consider supplier 1 and the second term in (4.18). Similar to (4.19) we have that

$$
\begin{aligned}
& \int_{\theta \in \Theta}\left[\tilde{x}^{1}(b(\theta))\left(v\left(q^{* 1}\left(\theta^{1}\right)\right)-\phi\left(q^{* 1}\left(\theta^{1}\right)\right)\right)+y^{1}(b(\theta))\right] \gamma(\theta) d \theta \\
= & \int_{\theta^{1} \in \Theta^{1}}\left[E_{-1}\left[\tilde{x}^{1}\left(b^{1}\left(\theta^{1}\right), b^{-1}\left(\theta^{-1}\right)\right)\right]\left(v\left(q^{* 1}\left(\theta^{1}\right)\right)-\phi\left(q^{* 1}\left(\theta^{1}\right)\right)\right)\right] \gamma^{1}\left(\theta^{1}\right) d \theta^{1} \\
& +\int_{\theta \in \Theta}\left[y^{1}(b(\theta))\right] \gamma(\theta) d \theta .
\end{aligned}
$$

Again, since the distribution of suppliers' bids under $b$ is by construction the same as under $\hat{b}$, the second term of (4.20) is not changing if we switch from $b$ to $\hat{b}$. That is

$$
\int_{\theta \in \Theta}\left[y^{1}(b(\theta))\right] \gamma(\theta) d \theta=\int_{\theta \in \Theta}\left[y^{1}(\hat{b}(\theta))\right] \gamma(\theta) d \theta .
$$

Also as before, the distribution of bids coming from the other suppliers (suppliers other than 1 that is) is by construction the same under both $b$ and $\hat{b}$. However, since $\hat{b}^{1}$ is not necessarily equal to $b^{1}$, we cannot guarantee that for all $\theta^{1} \in \Theta^{1}$

$$
E_{-1}\left[\tilde{x}^{1}\left(b^{1}\left(\theta^{1}\right), b^{-1}\left(\theta^{-1}\right)\right)\right]=E_{-1}\left[\tilde{x}^{1}\left(\hat{b}^{1}\left(\theta^{1}\right), \hat{b}^{-1}\left(\theta^{-1}\right)\right)\right] \cdot \cdot^{15}
$$

\footnotetext{
${ }^{13}$ Given that supplier 1 has mixed strategies under $\hat{b}^{1}, y^{i}(\hat{b}()$.$) already incorporates the expectation over supplier 1$ 's mixed strategies. The same applies to $y^{1}(\hat{b}()$.$) later in this section.$

${ }^{14}$ Given that supplier 1 has mixed strategies under $\hat{b}^{1}, E_{-i}[$.$] denotes the expectation taken over all type profiles \theta^{-i}$ as well as the corresponding mixed strategies of supplier 1 .

${ }^{15}$ Given that supplier 1 has mixed strategies under $\hat{b}^{1}, E_{-1}[$.$] denotes the expectation taken over all type profiles$ $\theta^{-1}$ as well as the corresponding mixed strategies of supplier 1 .
} 
Nevertheless, from Lemma 4.3 it follows that the set of types $\theta^{1} \in \Theta^{1}$ for which

$$
E_{-1}\left[\tilde{x}^{1}\left(b^{1}\left(\theta^{1}\right), b^{-1}\left(\theta^{-1}\right)\right)\right] \neq E_{-1}\left[\tilde{x}^{1}\left(\hat{b}^{1}\left(\theta^{1}\right), b^{-1}\left(\theta^{-1}\right)\right)\right]
$$

has Lebesque measure zero. Thus, we can still guarantee that the first term of (4.20) is not changing if we switch from $b$ to $\hat{b}$.

Based on the above analysis we find that

$$
U^{0}(b)-U^{0}(\hat{b})=0
$$

That is, the expected utility for the buyer in equilibrium $b$ is the same as in $\hat{b}$. Furthermore, changing the bidding strategies of the other suppliers $(2, \ldots, n)$ one by one and applying the above analysis yields that also

$$
U^{0}(b)-U^{0}(\tilde{b})=0
$$

That is, the expected utility for the buyer in equilibrium $b$ is the same as in $\tilde{b}$.

\subsection{Application}

The main result of Section 4.3 is the multi-item extension of Asker and Cantillon's (2008) result that pseudo-types are a sufficient statistic in quasi-linear, single item scoring auctions. They use their result to establish a link between single item scoring auctions and the well studied standard single item IPV (independent private values) auctions. They demonstrate how knowledge about these IPV auctions can be used for the equilibrium analysis in single item scoring auctions. Similar to them, we can establish a link between combinatorial scoring auctions and the regular, price-only combinatorial IPV auctions.

We observe that for every combinatorial scoring auction described in Section 4.2 there exists a related combinatorial price-only auction allocating $a$ items to $n$ bidders. For this price-only auction

- the allocation rule is $x$,

- the payment rule is $y=\left(y^{1}, \ldots, y^{n}\right)$ (see (4.7) for definition),

- bidder $i$ 's type is his pseudo-type and is distributed accordingly,

- bidder $i$ 's utility function is specified according to (4.8), that is, given a true type $t^{i}$ his utility 
for a profile of reports $s=\left(s^{1}, \ldots, s^{n}\right)$ is

$$
u^{i}\left(s \mid t^{i}\right)=\tilde{x}^{i}(s) t^{i}-y^{i}(s)
$$

(see right above (4.6) for the definition of $\tilde{x}^{i}$ ).

Note that a supplier's bidding strategy in the above price-only auction is a mapping $T^{i} \mapsto \mathbb{R}^{d}$. Every bidding strategy like this can be seen as a pseudo-type based bidding strategy $\Theta^{i} \mapsto \mathbb{R}^{d}$ specifying the same bid for all types yielding the same pseudo-type.

The above observation implies that, if allocation equivalent mixing is essentially absent, we can use the existing literature on combinatorial price-only auctions to analyze the equilibria in the scoring auction. In the following we illustrate this by constructing an efficient scoring auction based on probably the most famous combinatorial auction, the Vickrey-Clarke-Groves (VCG) mechanism (Vickrey (1961), Clarke (1971), Groves (1973)). However, note that the classic combinatorial auction literature has to offer much more than just the standard VCG auction, see Cramton, Shoham and Steinberg (2006) for an overview. A recent stream of literature for example is dealing with sufficient conditions for the existence of equilibria. Chapters 2 and 3 are part of this stream. A more detailed literature overview regarding this topic can be found in the corresponding introductory sections to these chapters. Another interesting stream of literature is concerned with ways to reduce the communication complexity and the computational complexity of the allocation algorithm in combinatorial auctions, see for example Holzman, Kfir-Dahav, Monderer and Tennenholtz (2004), Nisan and Ronen (2000), Bartal, Gonen and Nisan (2003) as well as Dobzinski, Nisan and Shapira (2006).

An additional example of the literature that can be used for the equilibrium analysis of combinatorial scoring auctions is the literature on bidder's utility in direct revelation mechanisms with multi-dimensional private information, see for example the revenue equivalence results in Chapter 3 as well as Krishna and Maenner (2001). Their results imply the following extension of Asker and Cantillon's (2008) "Expected Utility Equivalence"-Theorem for single item scoring auctions to combinatorial scoring auctions. Note that in the following we consider pseudo-type based equilibria where a supplier assigns the same scored bid to all types yielding the same pseudo-type. We are going to denote a supplier's bidding strategy as $b^{i}\left(t^{i}\right)$, that is directly as a mapping $T^{i} \mapsto \mathbb{R}^{d}$.

Corollary 4.1 Consider two scoring auctions $\left(S_{1}, \ldots, S_{d}, x, w\right)$ and $\left(S_{1}, \ldots, S_{d}, x^{\prime}, w^{\prime}\right)$ as well as a pair of corresponding pseudo-type based equilibria $b$ and $b^{\prime}$. Suppose that all pseudo-type spaces are convex. 
If

1) $x(b(t))=x^{\prime}\left(b^{\prime}(t)\right), \forall t \in T$ and

2) $U^{i}\left(b^{i}\left(t^{* i}\right) \mid t^{* i}\right)=U^{\prime i}\left(b^{i}\left(t^{* i}\right) \mid t^{* i}\right)$ for some fixed $t^{* i} \in T^{i}, \forall i \in N$,

then the equilibria $b$ and $b^{\prime}$ of the two auctions generate the same expected utility for the buyer.

Condition 1 asserts that both allocation rules and equilibria yield the same allocation vectors for each possible pseudo-type realization of the suppliers. Condition 2 states that both equilibria generate the same expected utility for supplier $i$ with fixed pseudo-type $t^{* i}$. In order to see why Corollary 4.1 holds, it is useful to observe that the buyer's expected utility (see also (4.11)) can be rewritten as

$$
\begin{aligned}
U^{0}(b)= & \int_{t \in T}\left[\sum_{i=1}^{n} \tilde{x}^{i}(b(t)) E_{\theta^{i} \in \Theta_{t^{i}}^{i}}\left[v\left(q^{* i}\left(\theta^{i}\right)\right)-c^{i}\left(q^{* i}\left(\theta^{i}\right), \theta^{i}\right)\right]\right] \pi(t) d t \\
& -\sum_{i=1}^{n} \int_{t^{i} \in T^{i}} U^{i}\left(b^{i}\left(t^{i}\right) \mid t^{i}\right) \pi^{i}\left(t^{i}\right) d t^{i},
\end{aligned}
$$

where $\pi^{i}$ denotes supplier $i$ 's density on $T^{i}, \pi$ denotes the joint density on $T$ and $c^{i}\left(q^{* i}\left(\theta^{i}\right), \theta^{i}\right)=$ $\left(c_{1}^{i}\left(q_{1}^{* i}\left(\theta^{i}\right), \theta^{i}\right), \ldots, c_{d}^{i}\left(q_{d}^{* i}\left(\theta^{i}\right), \theta^{i}\right)\right)$. The optimal quality levels chosen by a supplier, $q^{i *}$, depend only on his type and the scoring rules. It follows from Conditions 1 that the first part of (4.21) is the same for both equilibria. Remember (see also (4.10)) that supplier $i$ 's expected utility $U^{i}\left(b^{i}\left(t^{i}\right) \mid t^{i}\right)$ consists of his valuation for his expected allocation minus his expected payment. Given Condition 1, supplier $i$ 's expected allocations are the same for both equilibria. Furthermore, the revenue equivalence Theorem 3.5 in Chapter 3 asserts that supplier $i$ 's expected payments for both equilibria differ only by an additive constant. By Condition 2 this constant is fixed to equal zero. It follows hat $U^{i}\left(b^{i}\left(t^{i}\right) \mid t^{i}\right)$ is the same for both equilibria. Hence, the second part of (4.21) is the same for both equilibria as well.

\subsubsection{The VCG Scoring Auction}

The VCG scoring auction is specified by the scoring rules $\left(S_{1}^{*}, \ldots, S_{d}^{*}\right)$, the allocation rule $x^{*}$ and the winning score rule $w^{*}$. In the following we define these rules. First of all, the scoring rules (see (4.2)) reflect the buyer's valuation. That is, for all $j \in\{1, \ldots, d\}$,

$$
S_{j}^{*}\left(p_{j}, q_{j}\right)=v_{j}\left(q_{j}\right)-p_{j}
$$

Note that in this case the $j$ th element of a supplier's pseudo-type is the maximum level of welfare that this supplier can create by producing and subsequently selling the set of items $j$. Suppose suppliers 
have the pseudo-type profile $t$. The welfare generated by a profile of allocation vectors $\chi$ is

$$
W(\chi, t)=\sum_{i=1}^{n} \tilde{\chi}^{i} t^{i}
$$

where $\tilde{\chi}^{i}$ denotes supplier $i$ 's allocation vector $\chi^{i}$ without the element for the empty item set (see also right above (4.6) for a definition). ${ }^{16}$

Based on a reported profile of scores $s$, the allocation rule in the VCG scoring auction distributes items over suppliers in such a way that welfare is maximized. This yields for every supplier one set of items $j^{*}$ (possibly the empty set) that he is contracted to deliver. So, in his corresponding allocation vector $x^{* i}(s)$ we have that $x_{j^{*}}^{* i}(s)=1$ whereas $x_{j}^{* i}(s)=0$ for all $j \neq j^{*}$. Furthermore, $x^{*}(s)$ maximizes $W(., s)$. Denoting the maximal level of welfare achievable based on score profile $s$ by $W_{\max }(s)$, we have that

$$
W_{\max }(s)=\sum_{i=1}^{n} \tilde{x}^{* i}(s) s^{i} .
$$

The welfare generated by suppliers other than $i$ is

$$
W_{\max }^{-i}(s)=\sum_{h \neq i} \tilde{x}^{* h}(s) s^{h}
$$

Similarly to the above we define $\widehat{W}_{\text {max }}\left(s^{-i}\right)$ as the maximum level of welfare that can be achieved by the others without supplier $i$ being present.

The winning score rule $w^{*}$ is now defined as follows. For the item set that he is winning $\left(j^{*}\right)$, every supplier is contracted a score reflecting the marginal impact that his presence has on the welfare generated by all the others. For all other item sets the assigned score is zero. That is, given a report profile $s$,

$$
w_{j^{*}}^{* i}(s)=\widehat{W}_{\max }\left(s^{-i}\right)-W_{\max }^{-i}(s),
$$

\footnotetext{
${ }^{16}$ To see this, note that if $\forall j, \phi_{j}()=.v_{j}($.$) then the buyer utility in (4.9) reduces to$
}

$$
u^{0}(s \mid \theta)=\sum_{i=1}^{n} y^{i}(s)
$$

Adding the suppliers' utilities (given in 4.8) now yields

$$
u^{0}(s \mid \theta)+\sum_{i=1}^{n} u^{i}\left(s \mid \theta^{i}\right)=\sum_{i=1}^{n} \tilde{x}^{i}(s) t^{i}\left(\theta^{i}\right) .
$$

So welfare depends only on the suppliers' allocation vectors and pseudo-types. 
whereas $w_{j}^{* i}(s)=0$ for all $j \neq j^{*}$. Using (4.7) this implies that

$$
\begin{aligned}
y^{* i}(s) & =x^{* i}(s) w^{* i}(s) \\
& =\widehat{W}_{\max }\left(s^{-i}\right)-W_{\max }^{-i}(s) .
\end{aligned}
$$

In the VCG scoring auction described above, it is a dominant strategy for suppliers to make a scored bid that corresponds to their respective pseudo-type. That is, $b=\left(b^{1}, \ldots, b^{n}\right)$ with $b^{i}\left(\theta^{i}\right)=$ $t^{i}\left(\theta^{i}\right)$ is a dominant strategy equilibrium in the VCG scoring auction. This result can be obtained directly by observing that the combinatorial price-only auction that relates to the VCG scoring auction above (as described in the beginning of Section 4.5) is the renowned VCG mechanism. This mechanism efficiently allocates the items and charges bidder $i$ with a payment $y^{i}$ that corresponds to the impact that his presence in the auction has on the welfare generated by the others. It is a well-known result (see for example Ausubel and Milgrom (2006)) that in the VCG mechanism it is a dominant strategy for bidders to truthfully bid their type $t^{i}$.

For completeness we state again the rationale for this result. Suppose that the other bidders have a fixed report profile $s^{-i}$. Then, the utility of bidder $i$ with type $t^{i}$ for making a report $s^{i}$ is

$$
\begin{aligned}
u^{i}\left(s \mid t^{i}\right) & =\tilde{x}^{* i}(s) t^{i}-y^{* i}(s) \\
& =\tilde{x}^{* i}(s) t^{i}+W_{\max }^{-i}(s)-\widehat{W}_{\max }\left(s^{-i}\right) \\
& \leq W_{\max }\left(t^{i}, s^{-i}\right)-\widehat{W}_{\max }\left(s^{-i}\right) \\
& =\tilde{x}^{* i}\left(t^{i}, s^{-i}\right) t^{i}+W_{\max }^{-i}\left(t^{i}, s^{-i}\right)-\widehat{W}_{\max }\left(s^{-i}\right) \\
& =\tilde{x}^{* i}\left(t^{i}, s^{-i}\right) t^{i}-y^{* i}\left(t^{i}, s^{-i}\right) \\
& =u^{i}\left(t^{i}, s^{-i} \mid t^{i}\right) .
\end{aligned}
$$

The first and fifth equality follow from (4.8). The second and fourth equality follow from the definition of $y^{* i}$. The third equality follows from the definition of $x^{*}$. Thus, truthful reporting is indeed optimal.

However, note that the close link to the VCG mechanism does not only imply that the VCG scoring auction shares the same merits, like for example the implementation in dominant strategies (so a supplier does not need to know the types of the other bidders in order to play his dominant strategy). It also implies that the VCG scoring auction exhibits the same weaknesses, like the communication and computational complexity. A detailed discussion of the pros and cons of the VCG mechanism can be found in Ausubel and Milgrom (2006). 


\subsection{Conclusion}

In this chapter we extended the work of Asker and Cantillon (2008) about the analysis of single item scoring auctions with one-dimensional pseudo-types to a framework featuring multiple items and multi-dimensional pseudo-types. In doing this we face several technical difficulties and have to make simplifying assumptions about the pseudo-type mapping in order to overcome these problems.

First of all, we assume that if a set of pseudo-types has Lebesgue measure zero then the set of types yielding this set of pseudo-types also has Lebesgue measure zero. This property is not explicitly mentioned in Asker and Cantillon (2008) because they state their results in terms of equilibrium outcome functions rather than in terms of equilibrium bidding strategies as we do. In this case this property is not needed. However, if Asker and Cantillon (2008) would state their results also in terms of equilibrium bidding strategies, they would need this property of the pseudo-type mapping as well. We consider the question whether this property follows from other properties of the setting also for their framework as still open.

Second, we assume that the pseudo-type space $T^{i}$ is rich enough, so that for every $t^{i} \in T^{i}$ there exists a $\hat{t}^{i} \in T^{i}$ that is either component wise bigger or smaller than $t^{i}$. Furthermore, we require that any two $t^{i}, \hat{t}^{i} \in T^{i}$, where $t^{i}$ is componentwise smaller than $\hat{t}^{i}$, can be connected via a finite sequence of pseudo-types that is stepwise increasing. We have to invoke these technical properties in order to make the presented proof technique work. Though it is not nice that we have to make these assumptions, it is the best we can do at this moment. As usual, if one switches from a one-dimensional framework to a multi-dimensional framework, things become quickly very complicated.

Finally, we want to remark the following. In (4.15) we derive a monotonicity condition similar to the ones presented in the two foregoing chapters. This monotonicity condition together with the assumption of a "rich enough" pseudo-type space (see remarks above) is used to establish the result in Lemma 4.2. Together with Lavrič's (1993) Theorem 4.1 this Lemma is then used in the proof of Lemma 4.3 to establish that the expected allocation mapping is continuous a.e.. It would be interesting to see whether this can be shown to follow directly from the monotonicity condition in (4.15). However, at this point we do not investigate this question and consider it a topic for further research. 



\section{Bibliography}

Ahuja, R. K., T. L. Magnanti, and J. B. Orlin (1993). Network Flows - Theory, Algorithms and Applications. New Jersey: Prentice-Hall.

Archer, A. and R. Kleinberg (2008). Truthful germs are contagious: A local to global characterization of truthfulness. In Proceedings of the 9th ACM Conference on Electronic Commerce (EC'08), New York, pp. 21-30. ACM.

Asker, J. and E. Cantillon (2008). Properties of scoring auctions. RAND Journal of Economics 39(1), 69-85.

Asker, J. and E. Cantillon (2009). Procurement when both price and quality matter. Mimeo. Forthcoming RAND Journal of Economics.

Aumann, R. J. (1987). Game theory. In J. Eatwell, M. Milgate, and P. Newman (Eds.), The New Palgrave: A Dictionary of Economics, Vol. 2, pp. 460-482. London: Macmillan.

Ausubel, L. M. and P. Milgrom (2006). The lovely but lonely Vickrey auction. In P. Cramton, Y. Shoham, and R. Steinberg (Eds.), Combinatorial Auctions, pp. 17-40. Cambridge: MIT Press.

Bartal, Y., R. Gonen, and N. Nisan (2003). Incentive compatible multi unit combinatorial auctions. In Proceedings of the 9th Conference on Theoretical Aspects of Rationality and Knowledge (TARK'03), New York, pp. 72-87. ACM.

Berge, C. (1963). Topological Spaces. New York: Macmillan.

Bichler, M. (2000). An experimental analysis of multi-attribute auctions. Decision Support Systems 29(3), 249-268. 
Bichler, M. and Kalagnanam (2005). Configurable offers and winner determination in multi-attribute auctions. European Journal of Operational Research 160(2), 380-394.

Bikhchandani, S., S. Chatterji, R. Lavi, A. Mu'alem, N. Nisan, and A. Sen (2006). Weak monotonicity characterizes deterministic dominant-strategy implementation. Econometrica 74(4), 1109-1132.

Branco, F. (1997). The design of multidimensional auctions. RAND Journal of Economics 28(1), $63-81$.

Cachon, G. P. and M. A. Lariviere (1999). Capacity choice and allocation: Strategic behavior and supply chain performance. Management Science 45(8), 1091-1108.

Che, Y.-K. (1993). Design competition through multidimensional auctions. RAND Journal of Economics $24(4), 668-680$.

Chen-Ritzo, C.-H., T. P. Harrison, A. M. Kwasnica, and D. J. Thomas (2005). Better, faster, cheaper: An experimental analysis of a multiattribute reverse auction mechanism with restricted information feedback. Management Science 51(12), 1753-1762.

Clarke, E. H. (1971). Multipart pricing for public goods. Public Choice 11(1), 17-33.

Cramton, P., Y. Shoham, and R. Steinberg (2006). Combinatorial Auctions. Cambridge: MIT Press.

David, E., R. Azoulay-Schwartz, and S. Kraus (2002a). An English auction protocol for multiattribute items. In Revised Papers from the Workshop on Agent-Mediated Electronic Commerce IV, Designing Mechanisms and Systems (AAMAS'02), London, pp. 52-68. Springer-Verlag.

David, E., R. Azoulay-Schwartz, and S. Kraus (2002b). Protocols and strategies for automated multi-attributes auctions. In Proceedings of the 1st International Joint Conference on Autonomous Agents and Multi-Agent Systems (AAMAS'02), New York, pp. 77-85. ACM.

David, E., R. Azoulay-Schwartz, and S. Kraus (2003). Bidders' strategy for multi-attribute sequential English auctions with a deadline. In Proceedings of the 2nd International Joint Conference on Autonomous Agents and Multi-Agent Systems (AAMAS'03), New York, pp. 457-464. ACM.

David, E., R. Azoulay-Schwartz, and S. Kraus (2006). Bidding in sealed-bid and English multiattribute auctions. Decision Support Systems 42(2), 527-556. 
Dobzinski, S., N. Nisan, and M. Shapira (2006). Truthful randomized mechanisms for combinatorial auctions. In Proceedings of the 38th Annual ACM Symposium on Theory of Computing (STOC'06), New York, pp. 644-652. ACM.

Feng, J. (2008). Optimal mechanism for selling a set of commonly ranked objects. Marketing Science 27(3), 501-512.

Groves, T. (1973). Incentives in teams. Econometrica 41(4), 617-631.

Gui, H., R. Müller, and R. V. Vohra (2004). Dominant strategy mechanisms with multidimensional types. METEOR Research Memorandum 04/046, Maastricht University, Faculty of Economics and Business Administration.

Heydenreich, B., R. Müller, M. Uetz, and R. Vohra (2009). Characterization of revenue equivalence. Econometrica $77(1), 307-316$.

Holmström, B. (1979). Groves' scheme on restricted domains. Econometrica 47(5), 1137-1144.

Holzman, R., N. Kfir-Dahav, D. Monderer, and M. Tennenholtz (2004). Bundling equilibrium in combinatorial auctions. Games and Economic Behavior 47(1), 104-123.

Jehiel, P. and B. Moldovanu (2001). Efficient design with interdependent valuations. Econometrica $69(5), 1237-1259$.

Jehiel, P., B. Moldovanu, and E. Stacchetti (1999). Multidimensional mechanism design for auctions with externalities. Journal of Economic Theory 85(2), 258-293.

Jehle, G. A. and P. J. Reny (2001). Advanced Microeconomic Theory (2nd ed.). Boston: Addison Wesly.

Khuri, A. I. (2003). Advanced Calculus with Applications in Statistics (2nd ed.). New Jersey: Wiley.

Kirchkamp, O. and B. Moldovanu (2004). An experimental analysis of auctions with interdependent valuations. Games and Economic Behavior 48(1), 54-85.

Klemperer, P. (1999). Auction theory: A guide to the literature. Journal of Economic Surveys 13(3), 227-286.

Krishna, V. and E. Maenner (2001). Convex potentials with an application to mechanism design. Econometrica 69(4), 1113-1119. 
Lavrič, B. (1993). Continuity of monotone functions. Archivum Mathematicum (BRNO) 29(1), 1-4.

Malakhov, A. and R. V. Vohra (2004). Single and multi-dimensional optimal auctions - A network approach. Discussion Papers 1397, Northwestern University, Center for Mathematical Studies in Economics and Management Science.

Mas-Collel, A., M. D. Whinston, and J. R. Green (1995). Microeconomic Theory. Oxford: Oxford University Press.

Milgrom, P. (2004). Putting Auction Theory to Work. Cambridge: Cambridge University Press.

Milgrom, P. R. (2000). An economist's vision of the b-to-b marketplace. Perfect Commerce White Paper. Available at: http://www.perfect.com.

Monderer, D. (2008, February). Monotonicity and implementability. Mimeo. Available at: http://ie.technion.ac.il/Home/Users/dov0.html.

Müller, R., A. Perea, and S. Wolf (2005). Weak monotonicity and Bayes-Nash incentive compatibility. METEOR Research Memorandum 05/039, Maastricht University, Faculty of Economics and Business Administration.

Müller, R., A. Perea, and S. Wolf (2007a). Combinatorial scoring auctions. METEOR Research Memorandum 07/020, Maastricht University, Faculty of Economics and Business Administration.

Müller, R., A. Perea, and S. Wolf (2007b). Weak monotonicity and Bayes-Nash incentive compatibility. Games and Economic Behavior 61(2), 344-358.

Myerson, R. B. (1981). Optimal auction design. Mathematics of Operations Research 6(1), 58-73.

Nisan, N. (2006). Bidding languages for combinatorial auctions. In P. Cramton, Y. Shoham, and R. Steinberg (Eds.), Combinatorial Auctions, pp. 215-231. Cambridge: MIT Press.

Nisan, N. and A. Ronen (2000). Computationally feasible VCG mechanisms. In Proceedings of the 2nd ACM Conference on Electronic Commerce (EC'00), New York, pp. 242-252. ACM.

Roberts, K. (1979). The characterization of implementable choice rules. In J.-J. Laffont (Ed.), Aggregation and Revelation of Preferences, pp. 321-345. Amsterdam: North-Holland.

Rochet, J.-C. (1987). A necessary and sufficient condition for rationalizability in a quasi-linear context. Journal of Mathematical Economics 16(2), 191-200. 
Rockafellar, R. T. (1966). Characterization of the subdifferentials of convex functions. Pacific Journal of Mathematics 17(3), 487-510.

Rockafellar, R. T. (1970). Convex Analysis. Princeton, New Jersey: Princeton University Press.

Saks, M. and L. Yu (2005). Weak monotonicity suffices for truthfulness on convex domains. In Proceedings of the 6th ACM Conference on Electronic Commerce (EC'05), New York, pp. 286293. ACM.

Shostak, P. (1981). Deciding linear inequalities by computing loop residues. Journal of the ACM 28(4), 769-779.

Strecker, S. and S. Seifert (2004). Electronic sourcing with multi-attribute auctions. In Proceedings of the 37th Hawaii International Conference on System Sciences (HICSS'04), Track 7, Washington, pp. 70165.2. IEEE Computer Society.

Suyama, T. and M. Yokoo (2005). Strategy/false-name proof protocols for combinatorial multiattribute procurement auction. Autonomous Agents and Multi-Agent Systems 11(1), 7-21.

The Royal Swedish Academy of Sciences (2005). The Sveriges Riksbank prize in economic sciences in memory of Alfred Nobel 2005. Press Release. Available at: http://nobelprize.org.

The Royal Swedish Academy of Sciences (2007). The Sveriges Riksbank prize in economic sciences in memory of Alfred Nobel 2007. Press Release. Available at: http://nobelprize.org.

Vickrey, W. (1961). Counterspeculation, auctions and competitive sealed tenders. Journal of Finance $16(1), 8-37$.

Yoon, K. P. and C.-L. Hwang (1995). Multiple attribute decision making: An introduction. Sage University Paper Series on Quantitative Applications in the Social Sciences 07-104, Sage University. 



\section{Nederlandse samenvatting}

Iedere dag komen we in aanraking met strategische situaties waarvan de uitkomst afhangt van onze interactie met andere mensen. Denk bijvoorbeeld aan alledaagse situaties zoals het stemmen met collega's over waar te gaan lunchen, het bieden op een zeldzame Ramones plaat op Ebay of het spelen van een spelletje Risk met vrienden. Speltheorie probeert het gedrag van mensen in zulke strategische situaties wiskundig te modelleren.

Het onderwerp van dit proefschrift valt onder een deelgebied van de speltheorie genaamd mechanism design. Mechanism design is de tak van speltheorie die zich bezig houdt met het ontwerpen van de regels van het spel. Een spel kan verschillende uitkomsten hebben. Iedere participerende agent heeft een verzameling van mogelijke uitkomsten tot zijn beschikking waaruit hij kan kiezen. Hoe precies, wordt bepaald door een mechanisme dat de regels van het spel definieert. Zo'n mechanisme bestaat uit twee regels, een toewijzingsregel en een betalingsregel. De toewijzingsregel bepaalt de uitkomst van het spel, gebaseerd op de acties van alle agenten. De betalingsregel bepaalt op eenzelfde manier de uitbetaling die iedere agent moet doen of ontvangt.

Om het bovenstaande te illustreren bekijken we de veiling van een schilderij als voorbeeld. De agenten in dit veilingspel zijn de deelnemende bieders. Een mogelijke uitkomst van de veiling is bijvoorbeeld dat bieder $A$ het schilderij wint. Een andere uitkomst zou zijn dat bieder $B$ wint. De actie die een bieder kan ondernemen om de uitkomst van de veiling te beïnvloeden is het bieden van geld voor het schilderij. Gebaseerd op de biedingen van alle bieders bepaalt de toewijzingsregel wie het schilderij krijgt en de betalingsregel bepaalt hoeveel iedereen moet betalen.

De voorkeuren van een agent voor bepaalde uitkomsten worden uitgedrukt door middel van zijn waarderingsfunctie. Zijn type vertegenwoordigt de beschikbare informatie die belangrijk is voor zijn waardering van de verschillende uitkomsten. In het voorbeeld van de veiling van het schilderij kan het type van de agent bijvoorbeeld een weergave zijn van hoezeer hij het schilderij mooi vindt. In 
dit proefschrift richten we ons op direct revelation mechanisms, een speciale klasse van mechanismen. In een direct revelation mechanism is het aankondigen van een type de enige actie die een agent kan ondernemen. De strategie van een agent wordt dominant genoemd als het zijn nut maximaliseert voor iedere mogelijke combinatie van acties die door de andere agenten ondernomen worden. Een doel van de ontwerper van het mechanisme kan zijn om een direct revelation mechanism te ontwerpen dat dominant strategy incentive compatible is. Dit betekent het ontwikkelen van een mechanisme zodanig dat het eerlijk aankondigen van zijn eigen type een dominante strategie is voor iedere agent. In dit proefschrift bekijken we Bayes-Nash situaties. In deze situaties kennen agenten de types van de anderen niet maar is de verdeling van mogelijke types voor iedere agent publiekelijk bekend. Meer specifiek bekijken we Bayes-Nash incentive compatible direct revelation mechanisms. Dit betekent dat het waarheidsgetrouw rapporteren van zijn eigen type het verwachte nut van iedere agent maximaliseert, gegeven dat alle andere agenten ook waarheidsgetrouw rapporteren.

In een recente stroming van de literatuur bekijken onderzoekers de karakterisering van dominant strategy incentive compatible direct revelation mechanisms in termen van alleen de allocatie. Anders gezegd, ze willen precies die toewijzingsregels beschrijven waarvoor ze het bestaan van een betalingsregel kunnen garanderen die het resulterende mechanisme dominant strategy incentive compatible maakt.

In Hoofdstukken 2 en 3 passen we dit idee toe op Bayes-Nash incentive compatible mechanisms. In Hoofdstuk 2 bekijken we direct revelation mechanisms in situaties waar agenten onafhankelijk verdeelde, eendimensionale types en quasi-lineaire nutsfuncties hebben. Agenten mogen afhankelijke waarderingen hebben. We laten zien dat monotoniciteit, een voorwaarde op de toewijzingsregel, een noodzakelijke voorwaarde is voor Bayes-Nash incentive compatibility. Deze voorwaarde stelt dat de verwachte toename van waardering van een agent door het waarheidsgetrouw rapporteren van een type $t$ in plaats van het verkeerd rapporteren van een type $s$ minimaal even groot moet zijn als de verwachte toename door het verkeerd rapporteren van $t$ in plaats van $s$.

Om vast te stellen dat monotoniciteit voldoende is voor Bayes-Nash incentive compatibility gebruiken we een netwerk aanpak vergelijkbaar met die van Gui, Müller en Vohra (2004) en Saks en $\mathrm{Yu}(2005)$ in hun werk over dominant strategy incentive compatible mechanisms. Aangezien de beperkingen inherent in de definitie van Bayes-Nash incentive compatibility een natuurlijke netwerk interpretatie hebben, bouwen we complete gerichte grafen voor de ruimte van de types van de agenten. Om dit te doen associëren we een knoop met ieder type en zetten we een gerichte zijde tussen ieder geordend paar knopen. De lengte van de zijde die van de knoop geassocieerd met type $s$ naar de 
knoop voor type $t$ gaat is gedefinieerd als de kosten van manipulatie. Dat wil zeggen, het verwachte verschil in de waardering van een agent voor het waarheidsgetrouw rapporteren van $s$ in plaats van het verkeerd rapporteren van $t$. Monotoniciteit komt overeen met de afwezigheid van 2-cycli met negatieve lengte in deze grafen.

We laten zien dat een toewijzingsregel Bayes-Nash incentive compatible is, dan en slechts dan als de grafen boven beschreven geen cycli met negatieve lengte hebben. Dit resultaat is het BayesNash equivalent van een vondst van Rochet (1987) die laat zien dat dominant strategy incentive compatibility beschreven kan worden in termen van de afwezigheid van cycli met negatieve lengte in vergelijkbare grafen.

We laten zien dat we extra restricties moeten opleggen om te kunnen aantonen dat monotoniciteit voldoende is voor Bayes-Nash incentive compatibility. Zulke condities zijn decomposition monotonicity van de kosten van manipulatie of dat de waarderingsfuncties van de agenten voldoen aan niet-afnemende verwachte verschillen. Voor deze restricties kunnen we aantonen dat monotoniciteit voldoende is. Resultaten van Myerson (1981), Malakhov en Vohra (2004) en Feng (2008) voor BayesNash incentive compatible mechanisms volgen als speciale gevallen van onze resultaten. Hun setting kan worden gevouwen in ons kader, dat een bredere klasse van ruimtes van types en alternatieve vormen van afhankelijkheid tussen waarderingen van agenten toelaat.

Verder laten we zien hoe we bijbehorende betalingsregels voor Bayes-Nash incentive compatible toewijzingsregels kunnen construeren door gebruik te maken van de lengte van het kortste pad in de grafen van de agenten. Een toewijzingsregel voldoet aan revenue equivalence als alle betalingsregels die het Bayes-Nash incentive compatible maken dezelfde verwachte uitbetaling opleveren tot aan een additieve constante. We tonen aan dat revenue equivalence op gaat als de ruimtes van de types van de agenten convex zijn en hun verwachte waarderingen convex zijn in hun eigen types. Daarmee tonen we revenue equivalence aan onder dezelfde condities als Krishna en Maenner (2001).

In Hoofdstuk 3 breiden we de analyse van Hoofdstuk 2 uit naar situaties met multidimensionale type ruimtes. We volgen dezelfde netwerk aanpak als voorheen en houden de structuur van het hoofdstuk zo dicht mogelijk bij het vorige. In dit hoofdstuk moeten we de ruimtes van de types van de agenten beperkten tot convex en hun verwachte waarderingsfuncties tot convex in hun eigen types. We laten zien dat als de kosten van de manipulatie decomposition monotone zijn of de waarderingsfuncties voldoen aan niet-afnemende verwachte verschillen, dan monotoniciteit in combinatie met een integreerbaarheidsconditie zowel noodzakelijk als voldoende is voor Bayes-Nash incentive compatibility. Hoewel enkel monotoniciteit niet meer voldoende is, bereiken we nog steeds een karakterisering 
die puur gebaseerd is op de waarderingen en de toewijzingsregel. Karakteriseringsresultaten van Jehiel, Moldovanu en Stacchetti (1999), Jehel en Moldovanu (2001) en Müller, Perea en Wolf (2007b) volgens als speciale gevallen van onze resultaten. Wederom kunnen we revenue equivalence aantonen onder dezelfde voorwaarden als Krishna en Maenner (2001).

In Hoofdstuk 4 bekijken we scoring veilingen. In het voorbeeld van de veiling van het schilderij is er alleen competitie tussen de bieders via de prijs. In principe kondigt iedere bieder een prijs aan die hij wil betalen en wie het hoogste bod doet krijgt het schilderij. Echter, soms is de prijs van een voorwerp niet het enige dat van belang is en spelen niet-monetaire zaken ook een rol. Denk bijvoorbeeld aan verscheidene bouwbedrijven die strijden om een overheidscontract om een snelweg aan te leggen. Een belangrijk punt is natuurlijk de prijs. Een ander is bijvoorbeeld de tijd die nodig is om het project te voltooien. Een bedrijf kan bieden om de snelweg aan te leggen voor 20 miljoen binnen 6 maanden terwijl een ander bedrijf biedt om het te doen voor 15 miljoen maar binnen 9 maanden.

Een methode die veel gebruikt wordt voor de analyse van scoring veilingen is om de setting te transformeren in een van een standaard, price-only veiling. Dan kunnen bekende resultaten voor deze price-only veilingen toegepast worden. We doen dit voor een scoring veiling waarin verschillende items met meervoudige attributen toegewezen moeten worden. We laten zien hoe deze setting te transformeren tot een combinatoriële veiling die lijkt op de setting met lineaire waarderingen besproken in Sectie 3.4.1 van het vorige hoofdstuk. Hiermee breiden we het werk van Asker en Cantillon (2008) uit tot een kader met meervoudige items. Asker en Cantillon (2008) bekijken een scoring veiling waarin een enkel item met meervoudige attributen moet worden toegewezen. Zij transformeren dit tot een price-only veiling met een enkel item. 


\section{Curriculum Vitae}

Sascha Wolf was born on December 16, 1978 in Perleberg, Germany. He attended high school at Marie Curie Gymnasium Wittenberge from 1992 to 1998, obtaining a university qualifying degree (Abitur). Subsequently, he joined the German army for 10 months of compulsory military service. From 1999 to 2003 he studied Econometrics at Maastricht University. He spent the spring semester 2003 studying at the Grenoble Graduate School of Business, France, where he followed courses in the Master in Management program. In October 2003 he obtained his Master's Degree in Econometrics.

Sascha joined the Department of Quantitative Economics at Maastricht University in September 2003. He worked under the supervision of Prof. dr. Rudolf J. Müller and Dr. Ir. A. Perea y Monsuwé. The results of his research are presented in this thesis. 